UCRL-ID-131695-Rev1

\title{
Active and Passive Computed Tomography Mixed Waste Focus Area Final Report
}

H. E. Martz, G. P. Roberson, D. C. Camp, D. J. Decman, J. A. Jackson and G. K. Becker

November 6, 1998

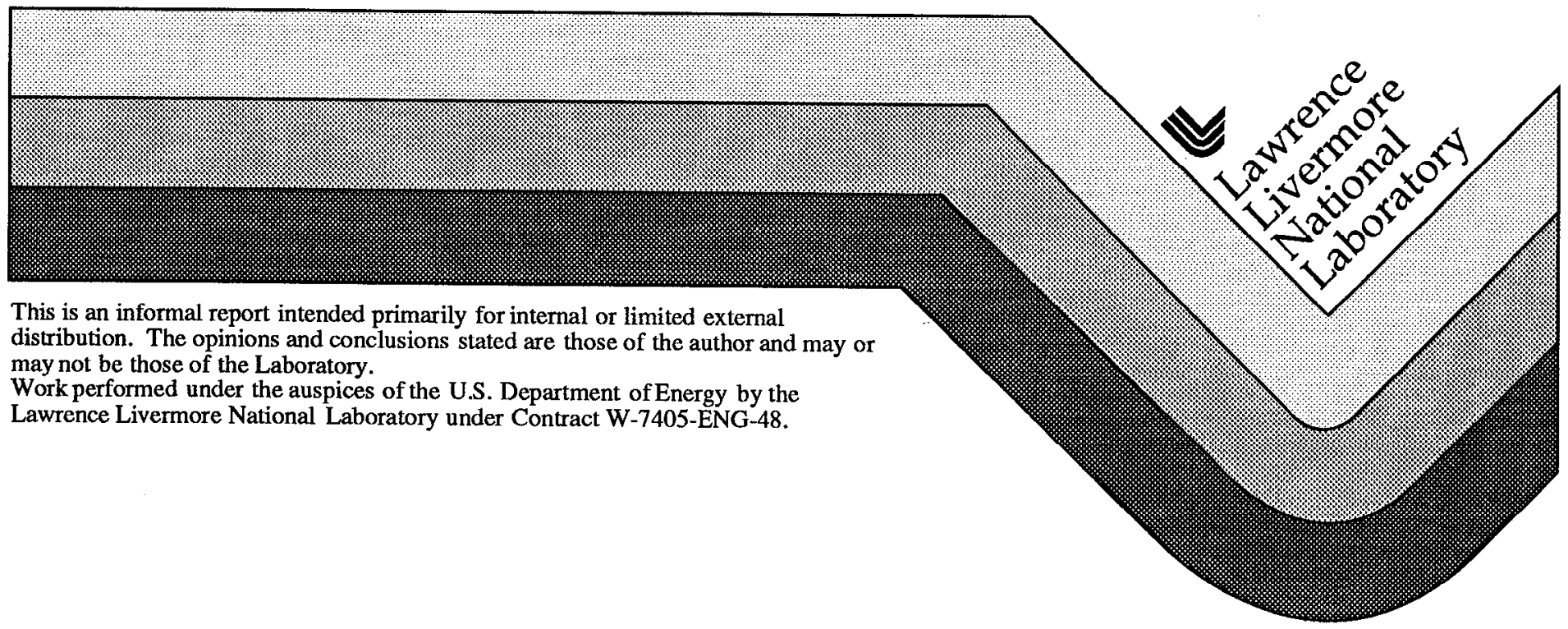




\section{DISCLAIMER}

This document was prepared as an account of work sponsored by an agency of the United States Government. Neither the United States Government nor the University of California nor any of their employees, makes any warranty, express or implied, or assumes any legal liability or responsibility for the accuracy, completeness, or usefulness of any information, apparatus, product, or process disclosed, or represents that its use would not infringe privately owned rights. Reference herein to any specific commercial product, process, or service by trade name, trademark, manufacturer, or otherwise, does not necessarily constitute or imply its endorsement, recommendation, or favoring by the United States Government or the University of Califomia. The views and opinions of authors expressed herein do not necessarily state or reflect those of the United States Government or the University of California, and shall not be used for advertising or product endorsement purposes.

This report has been reproduced directly from the best available copy.

Available to DOE and DOE contractors from the Office of Scientific and Technical Information P.O. Box 62, Oak Ridge, TN 37831

Prices available from (615) 576-8401, FTS 626-8401

Available to the public from the

National Technical Information Service

U.S. Department of Commerce

5285 Port Royal Rd.

Springfield, VA 22161 


\title{
Active and Passive Computed Tomography Mixed Waste Focus Area Final Report
}

\author{
Harry E. Martz, G. Patrick Roberson, \\ David C. Camp, Daniel J. Decman and Jessie A. Jackson \\ Lawrence Livermore National Laboratory \\ 7000 East Avenue, Mail Stop L-333 \\ Livermore, CA 94550 USA \\ and \\ Greg K. Becker \\ Lockheed Martin Idaho Technologies Company \\ Idaho National Engineering and Environmental Laboratory \\ Idaho Falls, ID, 83415 USA
}

November 6, 1998

Version 11.5 


\section{Table of Contents}

Table of Figures ..................................................................................... iv

Page No.

Table of Tables .....................................................................................

Executive Summary ............................................. vi

I. Introduction.............................................................. 1

I.A General Background............................................................................ 2

I.B Introduction to A\&PCT Technology ................................................. 4

II. Technology Description ......................................... 5

II.A Theory ....................................................................................... 5

II.A.1 Active Computed Tomography (ACT) ........................................ 6

II.A.2 Passive Computed Tomography (PCT) ......................................... 7

II.A.3 Coupling Active and Passive Computed Tomography.............. 8

II.B A\&PCT Technology ....................................................................... 9

II.B.1 A\&PCT Data Acquisition ........................................................... 9

II.B.1.1 Data Acquisition Hardware ...................................................... 10

II.B.1.2 Data Acquisition Software …................................................... 12

II.B.1.3 Data Acquisition Modes and Protocols ....................................... 13

II.B.2 Gamma-ray Spectral Analysis Software........................................ 14

II.B.2.1 Gamma-ray Spectral Analysis...................................................... 14

II.B.2.2 Self Attenuation Correction Method ......................................... 17

II.B.3 Image Reconstruction and Assay Determination......................... 18

II.B.3.1 Reconstruction Code Structure................................................ 19

II.B.3.1.1 Model Code ................................................................. 19

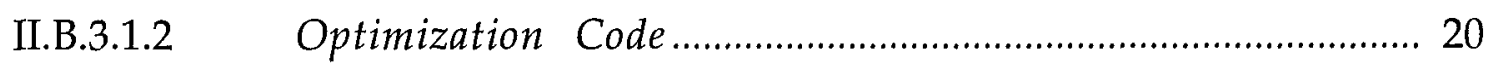

II.B.3.2 Code Development History …….................................................. 21

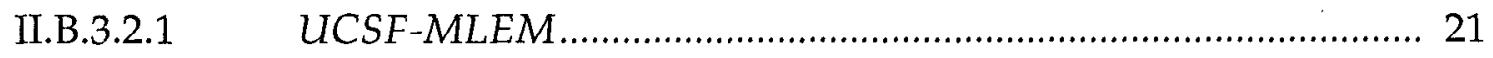

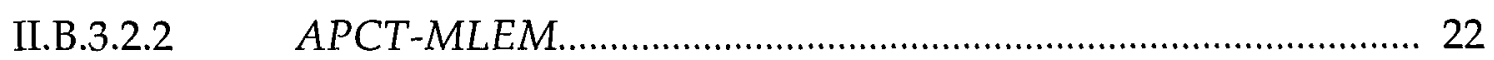

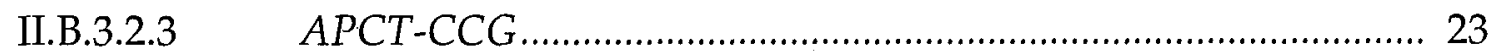

II.B.3.2.4 APCT-CCG with Measured Response Functions..................... 23

II.B.3.3 Results - Performance of Codes ................................................ 23

II.C Operational Configuration ………............................................ 31

II.C.1 IMPACT Configuration.......................................................... 31

II.C.1.1 Hardware .............................................................................. 31

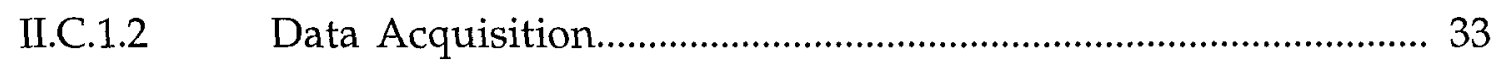

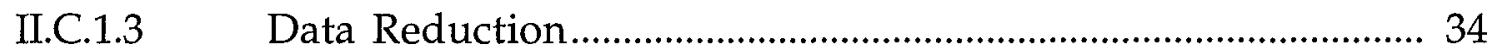

II.C.1.4 Calibration of IMPACT _........................................................... 34 


\section{Table of Contents - continued}

\begin{tabular}{|c|c|}
\hline II.C.1.5 & $\begin{array}{r}\text { Page } ~ \\
\ldots \ldots \ldots \ldots \ldots \ldots \ldots \ldots \ldots \ldots \\
\end{array}$ \\
\hline II.C.2 & WIT / A\&PCT Configuration.. \\
\hline II.C.2.1 & Hardware ..................... \\
\hline II.C.2.2 & Data Acquisition......... \\
\hline II.C.2.3 & Data Reduction.... \\
\hline II.C.2.4 & Calibration of WIT/A\&PCT \\
\hline II.C.2.5 & Validation of WIT/A\&PCT.... \\
\hline
\end{tabular}

III. A\&PCT Performance .................................................. 43

III.A Requirements and Performance Criteria........................................... 43

III.A.1 Quality Assurance Program Plan - Table 9.1................................... 44

III.A.2 Performance Demonstration Program........................................... 44

III.A.3 Capability Evaluation Project........................................................ 46

III.A.4 Rapid Commercialization Initiative ............................................... 48

III.B IMPACT Test Descriptions and Performance.................................... 49

III.C WIT/A\&PCT Test Descriptions and Performance ........................... 52

III.D Summary of A\&PCT Performance ………...................................... 56

IV. Implementation/Deployment Status ............................ 59

IV.A Demonstrated Capabilities ……………...................................... 59

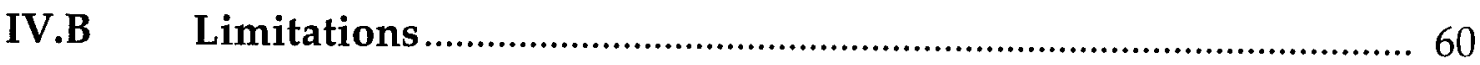

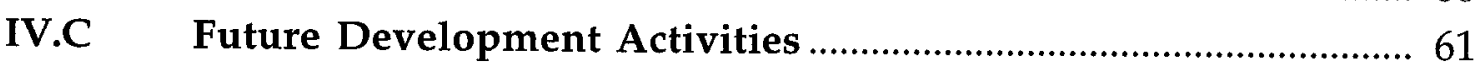

IV.C.1 Multiple Detector Development .................................................. 62

IV.C.2 Smart Scan Techniques .............................................................. 63

IV.C.3 Automated Isotopic Analyses ...................................................... 63

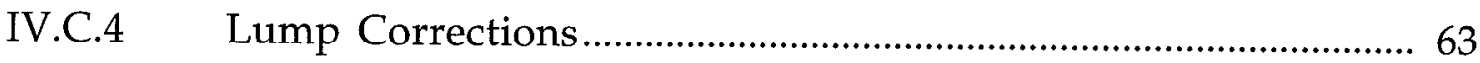

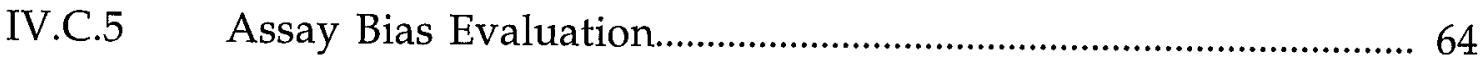

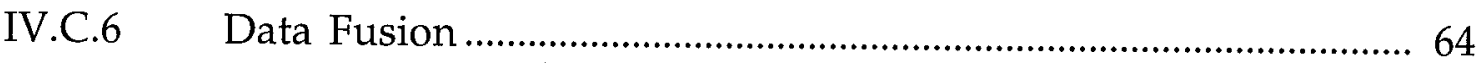

V. Acknowledgments................................................... 64

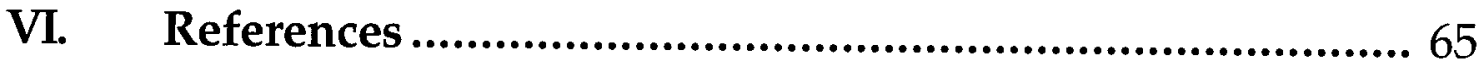

Appendix A .......................................................... 71 


\section{Table of Contents to Figures}

II.B.1-1 Four Major Components of an A\&PCT System................................. 10

II.B.3-1 Conceptual Design of a PCT Image Recon. Code................................ 19

II.B.3-2 Diagram of 3 Different Reconstruction Code Constructions.......... 22

II.B.3-3 Comparison of MLEM and CCG Optimization Methods................. 24

II.B.3-4 Comparison of 3 Sinogram images, Simulated Data ........................ 25

II.B.3-5 Fraction Recovered vs. Ray Sum Integration Time, PDP Data...... 26

II.B.3-6 Comparison of Meas. and Calc. Sinograms $17 \mathrm{sec}$. PDP Data.......... 28

II.B.3-7 Comparison of Meas. and Calc. Sinograms 160 sec. PDP Data........ 29

II.B.3-8 Comparison of Recon. Methods for Samples w/ Known Values. 30

II.B.3-9 Fraction Recovered vs. ${ }^{239}$ Pu gram amounts vs. Recon. Codes .... 30

II.C.1-1 Photo and Schematic of IMPACT at LLNL ..................................... 32

II.C.1-2 PCT Images of 3 Adjacent Slices for a Point Source............................ 37

II.C.2-1 Photo and Schematic of the BIR Inc., WIT Trailer ........................... 39

II.C.2-2 Schematic of a WIT Validation Drum Measurement ..................... 42

II.C.2-3 Schematic PCT Images of WIT Validation Run................................. 42

III.B-1 IMPACT Results vs. Ray Sum Acquisition Times for PDP002 _.... 49

III.B-2 Summary of IMPACT Precision - 15 replicate runs -PDP003........... 51

III.B-3 Summary of IMPACT Assay Results-PDP003 and PDP004 ............ 51

III.B-4 Three-Dimensional Rendered IMPACT Result - PDP Cycle 4 ....... 60

III.D-1 Accuracy Performance Results for IMPACT and WIT..................... 58

III.D-2 Precision Performance Results for IMPACT and WIT ................... 58

IV.C-1 A\&PCT Data Acq. Times for Future 6-HPGe Detector System....... 62 


\section{Table of Contents to Tables}

II.C.1.2-1 Results from the Assay of ${ }^{133} \mathrm{Ba}$ Results - MLEM Code

Page No.

II.C.1.2-2 Comparison of Recon Results Same Data for 3 Different Codes ... 38

III.A-1 NTP Quality Assurance Objectives for NDA Assay ..................... 45

III.A-2 Summary of PDP QAO Criteria............................................... 46

III.A-3 Measurement Precisions - 8 Replicates CEP Test ............................ 47

III.A-4 Bias QAO for the CEP taken from PDP .......................................... 48

III.C-1 WIT/A\&PCT Performance Total $\alpha$-Curie: CEP Test...................... 54

III.C-2 WIT/A\&PCT Performance: Total $\alpha$-Curie: RCI Test...................... 55

III.C-3 Radionuclides Identification in Waste by WIT ............................... 56

III.D-1 IMPACT \& WIT Blind Test Results-PDP \& Surrogate Drums..... 57

\section{Appendix}

A-1 Primary Radioisotopes Gamma-rays of Interest............................... 72

A-2 Rarely Encountered Radioisotopic Gamma Rays ......................... 72 


\section{Executive Summary}

A computed tomography (CT) and gamma-ray-based nondestructive assay technology has been developed that can identify and quantify all detectable radionuclides (with emphasis on transuranic, TRU, isotopes) in waste drums. The technology uses a radioactive source to simultaneously map on a voxel-byvoxel basis any number of selected monoenergetic gamma-ray attenuations for an entire waste drum. This mode is called active computed tomography or ACT. A second measurement without the ACT source, passively records in CT fashion the entire gamma-ray energy spectrum emitted from the drum also on a voxelby-voxel basis. The passive CT or PCT data are coupled with the ACT data to reconstruct and nondestructively assay (NDA) the contents of a radioactive waste drum. From these data radioactive waste content is identified and quantitative TRU gram amounts of detected isotopes are determined. Thus, a Pu-equivalent mass, an alpha-Curie value and total specific power can be determined for lowlevel, TRU and mixed-waste drums.

The active and passive computed tomography (A\&PCT) technology currently utilizes a single, high-purity germanium (HPGe) detector to collect gamma-ray spectra in both ACT and PCT data acquisition. This technique is very simply calibrated by recording only one measurement of the gamma-ray emissions from a NIST-traceable source at a known location within an empty drum. Once calibrated the A\&PCT measurements can be made of any drum with a known or unknown, homogeneous or heterogeneous waste matrix. No other information is required about drum content and no other calibration steps or matrix correction factors are necessary. In this technology and others measurement times depend on the density of the waste matrix and activity levels of the resident TRU isotopes. In our single-HPGe detector, proof-of-principle system the $\mathrm{ACT}$ and PCT measurement times varied from tens of hours each for dense sludge and/or matrices with low-gram TRU content to several hours each for matrices with high activity levels such as filters or low-density dry combustibles. In future multiple HPGe-detector A\&PCT systems, these measurement times will decrease by factors of from 10 to 40 or more.

Two A\&PCT systems were developed and tested. The first was located at the Lawrence Livermore National Laboratory (LLNL) and culminates a ten-year $R \& D$ effort to define the capabilities of this technology. The second A\&PCT system was developed over the past five years in cooperation with an industrial partner, Bio-Imaging Research, Inc., of Lincolnshire, IL. The latter system was constructed into a mobile trailer, a waste inspection tomography (WIT) laboratory, along with additional nondestructive evaluation (NDE) x-ray measurement modalities. This mobile laboratory provides a complete NDA and NDE capability that is transportable to any DOE site. Both systems employ radioactive transmission sources to enable monoenergetic ACT waste-matrix attenuation measurements; both use a HPGe detector for their ACT and PCT measurements; and both use a staging system to manipulate the drum or sourcedetector pair to enable the A\&PCT data to be acquired. WIT has been fully operational for the past two years. 
Results of two performance demonstration program (PDP) test measurements for the LLNL system using the UCSF-MLEM assay code show it to have a $-30 \%$ bias, but with precision at or below $1 \%$ for 15 replicate measurements. In addition, the LLNL system gave high-positive bias results for drums with low TRU content; however, this appears to be a result of reconstruction/assay codes that improperly handled statistical zero counts. Reanalysis of these data using improved passive CT reconstruction/assay codes (APCT-MLEM and APCT-CCG) reduced both biases. For drums with sufficient counting statistics the negative bias has been reduced to about $10 \%$.

The WIT system participated in three different blind performance test measurement programs: the rapid commercialization initiative ( $\mathrm{RCI})$; the capability evaluation project (CEP); and the PDP. Results in total bias (\% recovery) and precision (\% relative standard deviation) for these three tests of WIT/A\&PCT using the UCSF-MLEM assay code are 119 and not measured; 125 and 2.9; and 104 and 2.2, respectively. The WIT RCI test did not include replicate measurements thus precision was not measured. WIT's ${ }^{239} \mathrm{Pu}$ mass \%R and \%RSD are 132 and 2.3 , respectively, for known surrogate drums. These results are independent of matrix type and activity level. For ${ }^{239} \mathrm{Pu}$ content greater than $45 \mathrm{~g}$ the WIT / A\&PCT total bias in \% $\mathrm{R}$ is 101 ; therefore the overall bias of $+30 \%$ appears to be due primarily to the assay of low gram amount drums, which were assayed using insufficient data acquisition times.

The A\&PCT technology has demonstrated compliance with the National Transuranic Program quality assurance objectives through three separate blind performance tests using two different A\&PCT systems. Calibration of both systems is simple and fast $(\sim 2 \mathrm{hr}$.). It requires the measurement of only one NIST - traceable point source at a known distance from the detector. Most importantly this calibration does not require the use of any surrogate waste matrices or radioactive working reference materials.

Future work underway for the WIT system includes an upgrade from one HPGe detector to six. These six detectors coupled with an improved PCT scanning protocol will enable both the ACT and PCT measurements to be made from 10 to 40 times faster depending on activity level and matrices. An improved gamma-ray spectroscopy analysis code will enable almost all TRU isotopes, as well as other infrequently occurring waste radioactivity, to be identified. This improved code may reveal when self-absorbing lumps of TRU material, especially plutonium, are present. Finally, we look forward to beginning to "fuse" results from this gamma-ray-based CT technology with results from both the NDE x-ray-based and the NDA neutron-based measurements. Such "data fusion" should lead to a more complete characterization and more accurate assays for all types of waste-drum matrices. 


\title{
Active and Passive Computed Tomography Mixed Waste Focus Area Final Report
}

\author{
Harry E. Martz, G. Patrick Roberson, \\ David C. Camp, Daniel J. Decman and Jessie A. Jackson \\ Lawrence Livermore National Laboratory \\ 7000 East Avenue, Mail Stop L-333 \\ Livermore, CA 94550 USA \\ and \\ Greg K. Becker \\ Lockheed Martin Idaho Technologies Company \\ Idaho National Engineering and Environmental Laboratory \\ Idaho Falls, ID, 83415 USA \\ November 6, 1998 \\ Version 11.5
}

\section{Introduction}

The Mixed Waste Focus Area (MWFA) Characterization Development Strategy delineates an approach to resolve technology deficiencies associated with the characterization of mixed wastes. The intent of this strategy is to ensure the availability of technologies to support the Department of Energy's (DOE) mixed-waste, low-level or transuranic (TRU) contaminated waste characterization management needs. To this end the MWFA has defined and coordinated characterization development programs to ensure that data and test results necessary to evaluate the utility of non-destructive assay technologies are available to meet site contact handled waste management schedules.

Requirements used as technology development project benchmarks are based in the National TRU Program Quality Assurance Program Plan. These requirements include the ability to determine total bias and total measurement uncertainty. These parameters must be completely evaluated for waste types to be processed through a given nondestructive waste assay system constituting the foundation of activities undertaken in technology development projects. Once development and testing activities have been completed, Innovative Technology Summary Reports are generated to provide results and conclusions to support EM-30, -40 , or -60 end user or customer technology selection. The active and passive computed tomography non-destructive assay system is one of the technologies selected for development by the MWFA.

Lawrence Livermore National Laboratory (LLNL) has developed the active and passive computed tomography (A\&PCT) nondestructive assay (NDA) 
technology to identify and accurately quantify all detectable radioisotopes in closed containers of waste. This technology will be applicable to all types of waste regardless of their classification-low level, transuranic or mixed. Mixed waste contains radioactivity and hazardous organic species. The scope of our technology is to develop a non-invasive waste-drum scanner that employs the principles of computed tomography and gamma-ray spectral analysis to identify and quantify all of the detectable radioisotopes. Once this and other applicable technologies are developed, waste drums can be nondestructively and accurately characterized to satisfy repository and regulatory guidelines prior to disposal.

\section{I.A General Background}

During the past 50 years most DOE sites that support defense programs have had, and some continue to have, operations that process uranium (U) and/or plutonium (Pu). These processes include steps which produce very pure actinide products, but they also generate waste products. Since the $1960 \mathrm{~s}$ traditional material control and accountability Safeguards has focused on the measurement of nearly ideal samples containing tens of grams to kilogram quantities of actinides such as pure uranium or pure plutonium oxides, solutions or metals. The kinds of NDA technologies developed to measure these products included both gamma-ray-based instruments, such as the segmented gamma-ray scanner (SGS), and neutron-based active and passive counters.

In the late 1980s when it became important to DOE's Environmental Restoration and Waste Management Program to begin characterizing wastes, many laboratories chose to adapt existing safeguards techniques to measure the amount of transuranic isotopes found in waste drums. $\Lambda t$ first, this seemed appropriate; however, it soon became clear that these methods and their assumptions were not necessarily adequate for certain waste form configurations. Some laboratories then chose to develop hybrid instruments that attempted to correct for the waste matrix and source configuration effects. Unfortunately, these hybrid instruments yield accurate answers only if separate calibrations are carried out for all possible waste matrices in which the transuranic isotopes might be found. To do so becomes time consuming and very costly because surrogate waste matrices must be constructed to cover all possible heterogeneous combinations of wastes that might be encountered within the DOE's waste complex. It would be more cost effective for DOE to develop a technology that obtains assay results independent of waste drum content. This greatly reduces the number of calibration drums required.

Subsequently, it has become clear that the characterization of the generated wastes, which contain $\mathrm{U}, \mathrm{Pu}$ and other actinides, may require more than one technology as a function of waste type that can identify and quantify the radioactive content within waste containers such as 208-liter drums (55gallon) and other sizes. [QAP96] Preferably, as many waste containers as 
possible will be assayed non invasively, because the analysis cost per opened container is prohibitively expensive. Categories for wastes containing radioactivity include contact-handled TRU, low level (LLW), or mixed. DOE's major decontamination and decommissioning (D\&D) effort will also generate radioactive wastes that must be categorized as TRU, LLW, or mixed. Such wastes also exist within the nuclear power industry; [LEV95] ${ }^{1}$ thus, once the computed tomography technology and others are developed, they can be utilized to properly characterize all categories of radioactive wastes.

Some of the non-destructive waste assay capabilities needed to support the DOE's contact-handled mixed waste characterization are a function of the waste forms in inventory. These waste forms exhibit a number of variables that impact assay system response and must be taken into account in order to ensure valid measurements. Such variables include; matrix density, matrix elemental composition, matrix density distribution, radionuclide and isotopic composition, physical and chemical form of the radioactive material, and their distributions within the waste matrix. Certain combinations of these variables result in waste configurations within the assay capability of one or more of the existing NDA systems. Other combinations prevalent in the inventory are outside the assay capability of existing systems.

To a certain extent commonly employed gamma-ray-based NDA techniques rely on the assumption that the sample matrix and the activity are in a uniform configuration. In fact, waste drums are often heterogeneous in matrix and radionuclide material distribution, and span a wide range of composition and matrix type. Thus, NDA system errors are related to nonuniform measurement responses associated with unknown radioactive source spatial distributions and matrix heterogeneities. These errors can be reduced by imaging techniques that better measure the spatial locations of sources and matrix attenuations.[LEV95a, DUN97, \& EST94]

A key difference between our technology and the others being developed is that we sought to develop a technology that would measure the amount of detectable transuranic isotopes in any waste matrix with few or no assumptions. For example, we do not make any assumptions or need correction factors for any waste stream, nor do we calibrate for a particular waste stream. All waste streams are treated the same in all of our assay measurements. Furthermore, in order to address both the concerns of the National TRU Program and the levels of radioactivities found in medical and commercial reactor wastes, the technology would measure any detectable gram amount of material found within any type of waste matrix. The assay time would be adjustable to reflect the amount of radioactivity content in the waste and the density of the waste matrix, both of which affect assay time. The technology operates in a manner similar to polyenergetic, $x$-ray-based computed tomography, which is used in the medical community to image

\footnotetext{
${ }^{1}$ All references throughout the text are indicated by a bracketed alphanumeric, i.e., [REF98] and are included in Section VI References, page 65ff.
} 
the brain and gastro-intestinal regions of a human patient. Once calibrated the A\&PCT technology can fully account for measurement complications such as heterogeneities in the waste matrix and unknown distributions of different radioisotopes. No other calibration steps are necessary. Thus, the A\&PCT development and testing project will enhance the overall utility of waste assay.

In addition to our work, there are several development efforts under way for the NDA of waste. ${ }^{2}$ In the U.S. they include neutron techniques[HEN97, HOG97, HOL97], gamma-ray techniques[EST97, PRE95 \& GRE97], or a combination of these two [BEC97, PIC98]. In addition, other efforts at laboratories outside the U. S. include: [GOT91, REI92, KAW90 LEV95a, COU98]. Of these, two in the U.S. [PRE95, GRE97] and four outside [REI92, KAW90, LEV95a, COU98] are using a CT approach. The efforts in Germany, Hungary and France are behind ours, while the effort at Hitachi Energy Research has been completed. The LANL tomographic gamma-ray scanner (TGS) effort is at about the same stage as LLNL's development effort. The commercialization of LLNL's A\&PCT technology has been transferred to BioImaging Research, Inc. (BIR).[BER95, MAR95, ROB98]

\section{I.B Introduction to A\&PCT Technology}

Gamma-ray spectroscopy with high-energy resolution germanium detectors has been successfully used as a quantitative radioactive assay method for many years. The excellent energy resolution associated with the use of high purity germanium (HPGe) detectors allows for accurate radionuclide identification. The energy resolution provided by HPGe detectors increases the signal to noise relative to that from other scintillation detectors thereby enabling accurate peak areas (or counts) to be extracted from very complicated spectra. For point sources there are several methods available to relate these peak areas to absolute assay values.

However, for many sample-detector geometries the point source assumption is not valid and it is difficult to relate peak areas to absolute intensities without calibration sources of the same energy and geometry. This problem arises because of the difficulty in calculating the detector solid angle for extended sources; this is made even more complicated when sample selfabsorption is important. For sources that are roughly the same size as the detector there are methods that can relate the extended source efficiency to point source calibration data. However, these methods become less reliable as the source dimensions become much larger than the detector. This is the case for the problem we discuss here, i.e., the assay of 208- $L$ drums using a $0.2 L$ detector. Moreover, these conventional gamma ray spectroscopy methods are most applicable when the source is uniformly distributed in a homogeneous

\footnotetext{
${ }^{2}$ Several good references for the contempory research, development, application and implementation of NDA systems are given in the Proceedings of the 4th, 5th and 6th NDA/NDE Waste Characterization Conferences.[NDA]
} 
attenuating matrix. Real waste drums rarely, if ever, have completely homogeneous radioactive material distribution within a homogeneous attenuating matrix.

Gamma-ray spectroscopy techniques have been implemented to address the waste drum assay problem. One commonly applied technique is the LANL developed segmented gamma-ray spectrometry (SGS) technique. The SGS technique is an accepted gamma-ray method throughout the DOE for assaying radioactive materials contained in waste drums. The SGS technique measures spatially averaged gamma-ray intensities in 8 to 10 segments, i.e., horizontal disk-shaped slices of the drum. The average matrix attenuation value for each slice is measured by the transmission of an external source. These attenuation values are used to correct each section's average passive gamma-ray emitted intensity. The A\&PCT technology we are developing is a more refined technique that improves the imaging from 10 large segments to almost four thousand small volume elements or voxels.

The A\&PCT method allows the gamma-ray spectroscopy portion of the absolute assay problem to be broken down into a simpler analysis. The A\&PCT technology uses two separate measurements both made on a voxelby-voxel basis for the entire drum. The first is an active (or transmission) gamma-ray interrogation of a waste container such as a 208-liter $\mathrm{drum}^{3}$ by one or more external radioactive source(s); and the second is a passive (or emission) measurement of the radioactive source(s) within the drum. The results of these two measurements are combined to produce an attenuation corrected assay of the gamma-ray radioactivities in the drum as follows. The absolute detector efficiency can be directly related to calibration measurements of point sources. The solid angle is accounted for in the image reconstruction algorithm. The passive measurement localizes the radioactivity of interest into small size voxels $(5.08 \mathrm{~cm}$ on a side). The active transmission source measurement provides data for an attenuation correction for each reconstructed passive voxel. The absolute assay can then be obtained by adding the counts in each of the voxels of the reconstructed passive CT image. Also, since some radionuclides of interest emit gamma-rays of more than one energy, one can obtain additional checks on the image reconstruction and the absolute assay results.

\section{Technology Description}

\section{II.A Theory}

Conventional active or transmission CT scanners measure the effects of an object on an incident beam or "ray" that travels in a straight path. For

\footnotetext{
${ }^{3}$ Throughout the text we will refer to a drum instead of a container since the A\&PCT scanner was optimized for drums of about 200 liters volume. This does not imply that the A\&PCT technology cannot assay other containers, e.g., we have assayed containers from 4 ( 1 gallon) to 300 (83 gallons) liters in volume.
} 
example, in x-ray or gamma-ray CT, the data measured are the photon intensities of the incident beam, $I_{o}[S(E), L]$, and the transmitted beam, $I[S(E), L]$, that was attenuated by the object along each ray path, $L$, for a photon energy spectrum $S(E)$. (This is true to first order, ignoring the effects of gamma- and x-ray scattering.)

The quantity that is reconstructed in CT is the value of some function, $f[S(E), x]$, for some volume element, or voxel, at location $x=(x, y, z)$ within the object. The reconstruction algorithms require line integrals, also called ray sums, for many ray paths $L$, which are defined as

$$
g[S(E), L]=\int_{L} f[S(E), x] d u,
$$

where $d u$ is the incremental distance along $L$. For $x$ - and gamma-ray CT, these ray paths are determined from the intensity measurements using the Beer's law relationship:

$$
g[S(E), L]=\ln \left[\frac{I_{0}[S(E), L]}{I[S(E), L]}\right] .
$$

These ray sums over many paths are needed to reconstruct $f[S(E), x]$.

\section{II.A.1 Active Computed Tomography (ACT)}

Conventionally, industrial and medical CT use an x-ray machine source with a wide energy spectrum and a current-integrating detector that integrates the energy deposited by photons over all energies. The resultant attenuation image is given by

$$
f[S(E), x]=\int_{S(E)} \mu[\rho(x), Z(x), E] d E \quad \text { (polyenergetic), }
$$

where $\mu$ is the linear attenuation coefficient, which is a function of volume density, $\rho$, and the atomic number, $Z$. Note that the resultant attenuation is integrated over the entire energy spectrum. The A\&PCT nuclear-spectroscopybased drum scanner differs from the conventional scanners in that it discriminates between photons of different energies. In this case, the resultant image is given by

$$
f(E, \boldsymbol{x})=\mu[\rho(\boldsymbol{x}), Z(\boldsymbol{x}), E] \quad \text { (monoenergetic). }
$$

The results are thus a discrete quantitative measurement of the linear attenuation coefficient at one energy $E$; i.e., there is no integration over the energy spectrum $S(E)$.

The ray path, $L$, is simplified if we consider a single $2-\mathrm{D} x-y$ plane fixed along the longitudinal axis, $z$, of a waste drum. We can treat a single discrete $x$ - or gamma-ray beam in that plane as a line or ray path defined by $s$, the distance between the ray path and the $(x-y)$ origin, and $\theta$, the angle of the $s$ axis 
from the $x$ axis. The transmitted (or active) beam intensity $I(E, s, \theta)$ for this ray path at a fixed energy, $E$, is

$$
I(E, s, \theta)=I_{0}(E, s, \theta) \exp \left[-\iint \mu(E, x, y) \delta(x \cos \theta+y \sin \theta-s) d x d y\right],
$$

where $\mu(E, x, y)$ is the spatial distribution of the linear attenuation coefficients at energy, $E ; I_{0}$ is the intensity of the incident beam; and $\delta$ is the Dirac delta function. The equation for the ray path is $x \cos \theta+y \sin \theta-s=0$. As mentioned earlier the argument of the exponential is known as a ray sum, $g(E, s, \theta)$, and in this case it is equal to

$$
g(E, s, \theta)=\ln \left[\frac{I_{0}(E, s, \theta)}{I(E, s, \theta)}\right]=\iint \mu(E, x, y) \delta(x \cos \theta+y \sin \theta-s) d x d y,
$$

It is useful to note that the CT ray sum is analogous to a simple gamma-ray transmission gauge experiment.

The set of ray sums at all values of $s$ at a given elevation (slice) for a fixed $E$ and $\theta$ is called a "projection," and a complete set of parallel-beam projections at all $\theta$ (from 0 to $180^{\circ}$ or $360^{\circ}$ ) for a fixed $E$ is called a "sinogram." From measurements of $I$ and $I_{0}$, a complete ACT sinogram can be determined; and various methods have been devised to reconstruct $\mu$, the linear attenuation coefficient, with filtered backprojection (FBP) being the most common.[BUD79] Therefore, $\mu$ is the parameter determined by image reconstruction of the ACT measurements at a selected energy value and voxel. For a waste drum, the attenuation due to the drum's contents, whether heterogeneous or homogeneous, is accurately measured in the third dimension by measuring sinograms at different $z$ planes (or clevations) of the drum. Note that ACT does not measure the presence or identity of any radioisotope, source strength or activity within a waste drum. They are measured by passive computed tomography (PCT).

\section{II.A.2 Passive Computed Tomography (PCT)}

As stated, PCT is used to measure the identity and quantity of gamma-ray emitting radionuclides within a waste drum. The ray sum for passive or single-photon-emitted CT (sometimes called SPECT) imaging, $g \gamma(E, s, \theta)$, is defined by [BUD79]

$$
g_{\gamma}(E, s, \theta)=I_{e}(E, s, \theta)=\iint \mathbf{p}(E, x, y) a(E, x, y, s, \theta) \delta(x \cos \theta+y \sin \theta-s) d x d y,
$$

where $I_{e}$ are the counts measured at each ray sum position and

$$
a(E, x, y, s, \theta)=\exp \left[-\int_{x}^{\text {detector }} \int_{y} \mu\left(E, x^{\prime}, y^{\prime}\right) \delta\left(x^{\prime} \cos \theta+y^{\prime} \sin \theta-s\right) d x^{\prime} d y^{\prime}\right],
$$

is the half-line attenuation integral from the $(x, y)$ position to the detector position defined by $(s, \theta)$; and $\mathbf{p}(E, x, y)$ are the photons of energy $E$ emitted per unit volume per unit time for each voxel within a waste drum. 
A single-photon-emitted ray sum is the integrated radioisotope activity, modified by one or a multiple of exponential attenuations, along the path from the source position within the drum to the detector. The influence of the term $a(E, x, y, s, \theta)$ depends on the magnitude and distribution of the attenuations within a waste drum, which are typically large and nonhomogeneous for most energies emitted. To obtain the most accurate results from the PCT measurements, the energy-dependent attenuations must be determined from ACT measurements. The commonly used assumption of a constant attenuation coefficient (e.g., the SGS method) is inadequate for accurate measurements of inhomogeneous waste matrices.

\section{II.A.3 Coupling Active and Passive Computed Tomography}

Coupling the ACT and PCT modes allows accurate and quantitative attenuation corrections to be determined specific to the location of any radioactivity detected. That is, once the attenuation caused by the waste matrix and geometry of the CT scanner is accounted for, an accurate measurement of the emitted photons, $\mathbf{p}$, from a radioisotope is determined. The radioisotopic activity from a particular radioisotope gamma ray, $j$, is determined as follows:

$$
C_{j}(E)=\sum_{i} p_{i}(E),
$$

where $C_{i}(E)$ is the total photons per unit time obtained from the sum of all the voxels at energy $E$ for the reconstruction of the passive CT data corrected by the ACT attenuation map. Once the total photons are obtained, the activity, $A^{j}$, is obtained from

$$
A^{j}(m C i)=\frac{C_{j}(E)}{t \varepsilon(E) \beta_{j} k},
$$

where $t$ is the ray sum integration time, $\varepsilon(E)$ is the HPGe efficiency ${ }^{4}$ at the particular energy $E$ of the emitted gamma ray measured, $\beta_{i}$ is the branching ratio for this particular gamma ray, and $\mathrm{k}$ is the constant $3.7 \times 10^{7}$ disintegrations per second per milliCurie. Finally, the measured activity is converted to a specific gram value mass, $m$, using

$$
m_{j}(g)=\frac{A^{j}}{A_{s p}^{j}},
$$

where $m_{j}$ is the mass in grams of radioisotope $j$, that has a specific activity given by $A_{s p}^{j}$.

The one sigma standard deviation total measurement uncertainty, TMU, is given by the following equation:

$$
T M U=\sqrt{\sigma_{T B i a s}^{2}+\sigma_{R S D}^{2}},
$$

\footnotetext{
${ }^{4}$ This does not include the solid angle since it is accounted for in the image reconstruction and assay algorithm as discussed in Section II.B.3.
} 
where the bias, $\sigma_{T B i a s}$ is given by

$$
\sigma_{\text {TBias }}=\sqrt{\sigma_{\text {stat }}^{2}+\sigma_{\text {iso }}^{2}+\sigma_{\text {bias }}^{2}},
$$

and $\sigma_{R S D}$ is the appropriate relative standard deviation of the precision derived from the multiple replicate measurements for the activity range measured. In equation II.A-E13, $\sigma_{\text {stat }}$ is the statistical uncertainty in the gamma-ray peak measurement, $\sigma_{i s o}$ is the uncertainty in the isotopic ratio measurement and $\sigma_{\text {bias }}$ is the bias determined from tests in which assay measurements are made of surrogate waste drums containing known amounts of TRU. The protocols required for some of these measurements are defined in the QAPP [QAP96] or PDP [PDP97].

\section{II.B A\&PCT Technology}

In order to assay an unknown waste drum using gamma-ray active and passive CT three techniques are required. These techniques are: (1) Gammaray spectroscopy data acquisition in both the active $(\mathrm{A})$ and passive $(\mathrm{P}) \mathrm{CT}$ modes; (2) Analysis of the gamma-ray spectra acquired in both the A and P modes; and (3) Reconstruction of both the A and P images. It is useful to describe briefly the principles and issues associated with each of these techniques to better understand the challenges of the NDA of waste drums.

\section{II.B.1 A\&PCT Data Acquisition}

Our active and passive computed tomography technology employs a scanner that uses high purity germanium (HPGe) detectors and their associated electronics.[DEB88, MAR91, ROB91 \& ROB94] It differs from conventional transmission CT scanners in that it discriminates between photons of different energies. The quantity that is reconstructed in active CT is the linear attenuation coefficient $(\mu)$ value for some volume element, or voxel, at location $x, y$, and $z$ within a drum (see Eq. II.A-E4). The voxel size and clarity are defined by scan and image reconstruction parameters. For a waste drum, the attenuation due to its contents is accurately measured in three dimensions and displayed as a sequence of two dimensional images at different $z$ planes (or elevations) of the drum, or as a 3-D volume or surface rendered image. As noted above in Section II.A.1, active CT does not identify any isotope or measure the source strengths of any radioactivities within a waste drum.

Passive $\mathrm{CT}$ is used to measure and determine both the identity and the strength of radioisotope sources within a drum. The ray sum for passive or single-photon-emitted CT (sometimes called SPECT) imaging is the counts (see Eq. II.A-E7) measured in disintegrations (d) per unit volume per unit time of a source within a waste drum. Therefore, a single-photon-emitted ray sum is the integrated radioisotope activity, modified by one or a multiple of exponential attenuations, along the path from a source position within a drum to the detector. The function that is imaged for passive CT is the counts 
corrected for matrix attenuation at one or more energies for all detectable radioisotopes within a drum. The gamma-ray spectrometry detection equipment collects the entire energy spectrum for each integration point and the radioisotopes are identified by their characteristic peaks within the energy spectrum (see Section II.B.2).

\section{II.B.1.1 Data Acquisition Hardware}

The hardware required to perform both active and passive modes consist of four principle components. These are: (1) Active source: used to acquire the active attenuation map; (2) Energy discriminating detector: used in both the active and passive modes; (3) Staging system: for manipulating the drum or source and detector during data acquisition; and (4) Computer system: used to acquire data and control staging.

These four components are shown in Figure II.B.1-1. The active source is a radioactive gamma-ray source that typically provides multiple mono-energetic peaks from $180-\mathrm{keV}$ to about $1.3 \mathrm{MeV}$. These gamma-ray peaks do not need to be the same energy as the gamma-rays emitted from within a waste container because interpolation between any two nearest neighboring active peaks or extrapolation can be performed. The HPGe detector is very efficient and has a relatively large-collimated aperture. Typically, we have used a radioactive source of a few $\mathrm{mCi}$. The A\&PCT method requires a high-energy resolution detector for identification and quantification of all detectable radionuclides. Therefore, we use a high energy resolution and high detection efficient germanium detector and associated spectroscopy electronics.

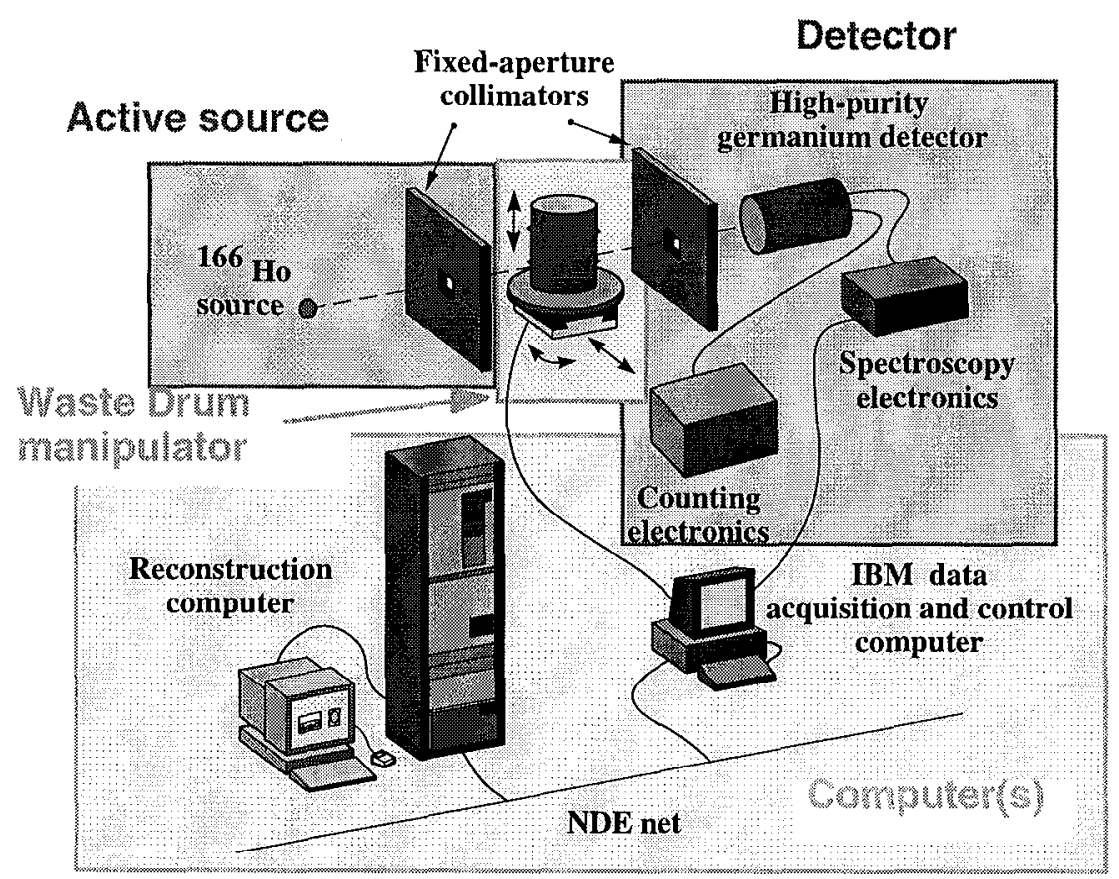

Figure II.B.1-1 The four major components (four shaded regions) of a nuclear-spectroscopy based active and passive tomography system. 
Two other important components of the A\&PCT scanner are the collimators for both the active source and detector. The apertures in these collimators are square. The detector-collimator aperture defines the spatial resolution (proportional to the voxel size) of the active-attenuation $\mathrm{CT}$ image and the corrected-passive CT image. The source collimator is used to direct radiation towards the detector only thus minimizing radiation that is not required in the active portion of the assay process. This ensures better data (reduced scatter), provides a safer operating environment, and eliminates any cross-detector transmissions when multiple transmission sources are used with multiple detectors.

Through simulation we studied the trade-off between spatial resolution and signal-to-noise.[KET95] Further, an optimum system design is dependent on expected emission source distribution and activity. Results of our simulation work revealed that A\&PCT systems with collimated square apertures from 2.5 to $7.5 \mathrm{~cm}$ on a side will perform best. In order to ensure that the detector fills the entire aperture area we need a large diameter detector. The largest diameter detectors with high counting efficiency are about $8.2 \mathrm{~cm}$ diameter. A large detector aperture reduces the assay time in two ways. First, a larger aperture utilizes more detector surface area providing a larger solid angle; second, it yields the advantage of fewer measurement positions (less sampling). Finally, we found that the aspect ratio (aperture length divided by aperture width) performs best if it is in the range of $5: 1$ to 10:1.[KET95] The smaller the aperture aspect ratio, the closer the detector will be to the waste drum; hence, the higher the counting rate because of the larger solid angle subtended. It is important to note that this has to be traded off with the spatial resolution required to get accurate assay results. For example, an improvement of a factor of two in spatial resolution in all dimensions requires a measurement duration at least eight times longer.

Data are acquired by either manipulating the drum, or manipulating the drum and source/detector pair. If only the drum is manipulated, the staging system must be capable of translating, elevating, and rotating the drum. For other cases, the source/detector pair may translate and/or elevate instead of the drum. Typically, for a single detector A\&PCT system, either the waste drum or the source/detector pair is discretely translated by a distance equal to the detector collimator's horizontal aperture dimension. For a single-detector system, the translation is performed for all ray sums required. If there were a sufficient number of detectors to cover a full transverse section of the drum, there would be no need to translate the drum. Thus, only drum rotation and elevation would be required for an assay.

The computer systems shown schematically in Figure II.B.1-1 are used to control the staging system, acquire data from the spectroscopy electronics, perform pre-processing functions, reconstruct data, and determine the final assay. The reconstruction process is discussed in Section II.B.3. The A\&PCT systems typically use PC computers to acquire data. After data acquisition, the 
pre-processing and reconstruction and assay processes can be performed on any computer such as a $\mathrm{SUN}^{5}, \mathrm{SGI}^{5}$ or PC.

\section{II.B.1.2 Data Acquisition Software}

The CT data acquisition software is written in C-code and consists of: (1) Input/output interface; (2) Stage control; (3) Data acquisition and digitization; and (4) Data preprocessing. The following paragraphs discuss each of these considerations in turn.

The input/output interface provides a window for entering the required scan parameters and other pertinent information about each CT scan. This information is stored on the disk in a CT parameter file in text format. This CT parameter file accompanies the data through the acquisition, reconstruction and assay reporting processes. All of the software programs associated with these processes must accept the CT parameter file's format. One CT parameter file is generated for each energy region of interest for both the projection data set and the reconstruction data set. The CT parameters are divided into several categories within the file and include information on: each scan, projection, active radiation source, detector and source geometry and collimators, HPGe detector specifications, scan-time parameters, reporting parameters, simulation (if applicable), and reconstruction. Some parameters are specific to the individual scanner that produced the data.

Stage control requires communication between the acquisition computer and the motor controller. Communication is usually accomplished through an RS232 port on the acquisition computer. The staging control subroutines in the data acquisition program typically vary depending on the manufacturer of the staging system being used and the CT scan geometry.

Data acquisition and digitizing subroutines depend on the detector system being used in the CT scanner. We acquire data from a single high-purity germanium detector where the sum of all interactions within the HPGe detector is digitized. The magnitude of the digitized signal is related to the energy of the sum of the photon interactions detected.

Each individual CT scanner dictates specific preprocessing methods and the number of preprocessing steps required as a result of the differing physical characteristics (i.e., source and detector systems used). The main preprocessing procedure consists of calculating the ray sums from the raw gamma-ray counts. Incident and transmitted counts are used to calculate ACT ray sums (see Eq. II.A-E6). These ray sums are used as input to the ACT image reconstruction codes. For PCT the emitted counts for all ray sums are combined with the attenuation images (see Eqs. II.A.-E6 and II.A-E7) to produce attenuation corrected PCT images of photons per unit time per voxel. These images are used to obtain a specific isotopic activity and mass using Eqs. II.A-E9 through E11.

\footnotetext{
${ }^{5}$ This does not imply that LLNL endorses either product or vendor.
} 
There are three important properties in acquiring both active and passive ray sums. First, the geometry of the ray paths (i.e., the source and detector positions in the object coordinate system) must be completely known; second, at any given geometric position, the incident and transmitted counts must be accurately measured and recorded during $\mathrm{ACT}$, and the emitted counts for a specific energy gamma ray or the entire spectrum must be accurately measured and recorded during passive CT; and third, for multiple-HPGe detector scanners, all preprocessed detector responses to a given energy and intensity must be identical; therefore, the responses from multiple HPGe detectors must be normalized to one value for all of the detectors.

\section{II.B.1.3 Data Acquisition Modes and Protocols}

We have two main protocols or modes of operation. These are collimated gamma-ray scanning (CGS) and A\&PCT. The CGS protocol is used to quickly determine the location of radioactivity with respect to height within a drum and to determine the data acquisition scan parameters (e.g., number of slices and scan time) required. The A\&PCT protocol is used to accurately assay the radioactivity within a waste drum. Both the CGS and the A\&PCT protocols employ the active and passive modes of drum scanning.

In the CGS protocol gamma-ray data is integrated while the drum is continuously rotated for each slice in both the active and passive drum scan modes. A full drum requires 18 slices. In the active mode the transmission source is opened and data for specific energy regions of interest (EROI) are obtained. In the passive mode, the transmission source is shuttered and selected EROIs or the entire gamma-ray spectrum is recorded.

In the A\&PCT protocol a set of transverse ray sums at one angle (a projection) are acquired at all translational positions until the drum diameter has been traversed. After each projection is acquired, the drum is rotated slightly for the next projection acquisition. In the active mode, projections are acquired over $180^{\circ}$, and in the passive mode they are acquired over $360^{\circ}$. Once a full set of projections are acquired, the drum or the source-detector pair is elevated by a distance equal to the vertical dimension of the detector's collimator aperture. This vertical dimension is the slice thickness. For a full drum, 18 slices are acquired for both the active and passive modes. All of the slice projections are used to reconstruct an image of the waste drum's attenuation (ACT) and emissions (PCT).

During an ACT data acquisition drum scan, EROIs are set for each of the major peaks of the transmission source since these are known. Data are collected from each EROI simultaneously for each ray sum acquired. The active data that are saved and used in the reconstruction process are the net counts, which are the gross counts in the full-energy energy peak at each EROI selected minus its background.

In passive drum scans, the entire energy spectrum is acquired for each ray sum. This differs from the active mode because the energy peaks emitted 
from within a waste drum are unknown. All of the emission spectra are used in one or more of several ways to evaluate the isotopic content of the waste drum's content (see next section).

\section{II.B.2 Gamma-ray Spectroscopy Analysis Software}

The A\&PCT data sets consist of hundreds of high-energy resolution gamma-ray spectra, one for each ray sum acquired. Generally, the statistics in each individual ray sum spectra are poor, but they can be summed to produce better quality spectra that can be analyzed by traditional gamma-ray spectroscopic analysis codes. Such an analysis can determine most of the radioactive isotopes present. This includes isotopes important to transuranic waste characterization, and isotopes that may be of interest to neutron-based NDA technologies or gamma rays that might interfere with the analysis.

The analysis of the passive CT gamma-ray spectra also yield the mass ratios of the important plutonium isotopes. The A\&PCT method could, in principle, determine a mass (see Eq II.A-E11) for each of these isotopes by reconstructing the sinograms of their characteristic gamma-rays in the spectrum. However, that is rarely possible due to their poor statistics. Instead the A\&PCT assay only needs to determine the mass of the most abundant TRU isotope, which is usually but not always ${ }^{239} \mathrm{Pu}$. Analysis of the spectra is used to determine other isotopic ratios. This information is used to calculate the thermal power, the total alpha-Curie activity, and the fissile gram equivalent necessary for the characterization of the waste.

The A\&PCT data acquisition code creates spectra in a binary format. We have developed a computer code that processes each of the ray-sum spectra. The code is written in standard FORTRAN and currently is implemented to run in MS Windows-NT on personal computers. Our gamma-ray analysis code reads these spectra and builds a summed binary spectrum as well as an ASCII spectrum data file. The actual spectral analysis is performed on this ASCII file which is saved for future analysis. In addition to forming the summed spectrum the code also analyzes each ray sum for all of the EROIs from a user-defined table. The results of this analysis provide gross and background counts from each EROI for each ray-sum. The resulting sinograms are then stored in a binary format used by the image reconstruction and assay code.

\section{II.B.2.1 Gamma-ray Spectral Analysis}

The gamma-ray spectroscopy analysis of the summed spectral data begins by fitting a universal background to the entire spectrum. The background for each channel in the spectrum is determined by a "peak erosion" technique; and this background is subtracted from the spectrum. Then, a standard peaksearch algorithm is used to find peaks. All pcaks determined are analyzed for centroid and area, and subsequently their energies are compared to a userdefined look-up table of known gamma-ray lines, primarily $\mathrm{Pu}, \mathrm{Am}$ and $\mathrm{U}$. 
Obviously, the spectrum's gain and zero (specified in a setup file) have to be well known for this comparison to be valid. In this first step, only individual peaks are checked against the known list. No detailed analysis is performed on peak multiplets. The peak search criteria are intentionally set loose so that some statistical anomalies are detected. This assures finding nearly all of the easily determined peaks. Background radiation isotopes (e.g., ${ }^{40} \mathrm{~K}$, Th-U daughter products) are found in this step, in addition to those isotopes of specific interest. From the preliminary analysis, data are flagged for further analysis if there are $\mathrm{U}, \mathrm{Pu}$ or Am gamma-ray peaks detected. At the present time, if no peaks from these three isotopes are detected, then the spectra is either a calibration spectrum or a spectrum that requires special analysis and is so flagged.

To determine accurate peak areas the full energy peaks must be separated from the background and Compton continuum. X-ray and gamma-ray peak shapes differ. Thus one must have a mathematical description of both shapes as a function of energy. The peak and background fitting algorithms can vary the appropriate peak-shape parameters in order to obtain the best fit. The best fit is defined as the minimum least square difference between the data points and their calculated values. One must also have a list of known peak energies and their respective branching ratios for all gamma rays of interest.

Currently, we examine each summed gamma-ray spectrum for the following TRU isotopic mass ratios: ${ }^{238} \mathrm{Pu} /{ }^{239} \mathrm{Pu},{ }^{240} \mathrm{Pu} /{ }^{239} \mathrm{Pu},{ }^{241} \mathrm{Pu} /{ }^{239} \mathrm{Pu}$, ${ }^{235} \mathrm{U} /{ }^{239} \mathrm{Pu}$, and ${ }^{241} \mathrm{Am} /{ }^{239} \mathrm{Pu}$. These ratio pairs are determined by analyzing closely spaced multiplets of gamma-ray lines emitted from these isotopes. In each case we use the known energies and branching ratios to fix as many parameters as possible in the least squares fit. The goal in the calculation is to generate a section of the spectrum mathematically from a set of peak shape parameters and intensities that most closely approximate the measured net signal. This best fit gives a minimum reduced Chi squared difference between the calculated signal and the measured data.

To find the best fit, the equations describing the various peaks are approximated by using a first order Taylor's series expansion about the trial values of the free parameters, which are adjusted to minimize differences between the equations and data. These adjustments are found by performing non-linear least-square iterations on the equations. This procedure is also known as the Gauss-Seidel or Newton-Raphson method. For rapid convergence the closer the initial choices are to the real values, the quicker the fitting process converges. One output of the fitting process is the best peak height of each isotopic peak. These are used to find the best measurement of the isotopic composition of an emission source. We also correct the extracted peak areas for efficiency and a general attenuation correction. These corrections can be important when the energy range of the analysis is more that $10 \mathrm{keV}$. 
Presently, we analyze data in seven regions of the gamma-ray spectrum:

- The region between 120 and $135 \mathrm{keV}$ is analyzed to determine the ${ }^{241} \mathrm{Am} /{ }^{239} \mathrm{Pu}$ ratio. This region contains six peaks, but the analysis is determined by the ratio of the ${ }^{239} \mathrm{Pu}$ peak at $129.3 \mathrm{keV}$ and the peak at $125 \mathrm{keV}$ from ${ }^{241} \mathrm{Am}$. However, all peaks in this region must be included in the fit to obtain a good analysis. This region gives a good indication of any excess americium in a drum as has been demonstrated by data collected at the Stored Waste Experimental Pilot Plant (SWEPP) located at the Idaho National Engineering and Environmental Laboratory (INEEL) and subsequently analyzed by our code.

- The region from 135 to $155 \mathrm{keV}$ is used to determine the ${ }^{241} \mathrm{Pu} /{ }^{239} \mathrm{Pu}$ ratio by comparing the $148.5-\mathrm{keV}\left({ }^{241} \mathrm{Pu}\right)$ to the $144.2-$ $\mathrm{keV}$ transition of ${ }^{239} \mathrm{Pu}$. This analysis can be complicated by the presence of ${ }^{235} \mathrm{U}$ and its transition at $143 \mathrm{keV}$. Similarly, the $152-$ $\mathrm{keV}$ peak of ${ }^{238} \mathrm{Pu}$ can also be analyzed; however, for weapons grade $\mathrm{Pu}$ this is a rather weak transition which greatly limits the accuracy of the derived ${ }^{238} \mathrm{Pu} /{ }^{239} \mathrm{Pu}$ ratio.

- The analysis of the $160-\mathrm{keV}$ region should give the best information about the ${ }^{240} \mathrm{Pu} /{ }^{239} \mathrm{Pu}$ ratio. The doublet of strong transitions from these two isotopes at $160 \mathrm{keV}$ can be a clear indication of the degree of "burn-up" in the plutonium. Unfortunately, this region is frequently contaminated by high Compton background in typical waste drum spectra, which limits the accuracy achievable.

- The 208-keV transition is very prominent in the gamma-ray spectra of the A\&PCT systems. This transition is from the decay of ${ }^{237} \mathrm{U}$ to ${ }^{237} \mathrm{~Np}$, the daughter of ${ }^{241} \mathrm{Am}$ and granddaughter of ${ }^{241} \mathrm{Pu}$. However, because of its higher specific activity, ${ }^{241} \mathrm{Pu}$ dominates in most cases. This peak can be compared to the 203keV peak of ${ }^{239} \mathrm{Pu}$ for another determination of the mass ratios. If ${ }^{235} \mathrm{U}$ is present its $205-\mathrm{keV}$ peak is compared to the $208-\mathrm{keV}$ peak to obtain its relative abundance.

- The regions near $340 \mathrm{keV}$ and at $375 \mathrm{keV}$ give additional measurements of the ${ }^{241} \mathrm{Pu} /{ }^{239} \mathrm{Pu}$ and ${ }^{241} \mathrm{Am} /{ }^{239} \mathrm{Pu}$ ratios.

- The $414-\mathrm{keV}^{239} \mathrm{Pu}$ transition is used in the PCT analysis. This region is also analyzed to look for possible contributions from ${ }^{237} \mathrm{~Np}$, which may be present in addition to the $\mathrm{Pu}$ isotopes.

- The region above $600 \mathrm{keV}$ contains peaks from ${ }^{239} \mathrm{Pu},{ }^{240} \mathrm{Pu}$, and ${ }^{241} \mathrm{Am}$ and can be very useful in some cases. The high energies of these transitions allow them to escape from highly attenuating 
waste matrices. However, branching ratios for these peaks are less than those for the lower energy regions so that the statistical quality of data for low waste-drum gram amounts is a problem.

The results of the analysis of these regions is then combined into a report of the mass ratios. Subsequently, the data are combined with the assay data for ${ }^{239} \mathrm{Pu}$ (or ${ }^{235} \mathrm{U}$ ) to calculate the desired waste characterization parameters. This report lists the energies and intensities of the gamma rays found in a spectrum along with their identification when possible. Those peaks not found to agree with any listed in the table are flagged as unknowns. In the future, we intend to implement an option that if no isotopes from these three major elements $(\mathrm{U}, \mathrm{Pu}, \& \mathrm{Am})$ are found, the program will continue to search for other TRU isotopes that are identified as important to the neutron-based NDA techniques and to the WIPP WAC (e.g., ${ }^{137} \mathrm{Cs}$, et al., see section III.C and Appendix A).

\section{II.B.2.2 Self-Attenuation Correction Method}

Transuranic materials that are in a physical form other than diffuse distributions, e.g., greater than 500- $\mu \mathrm{m}$ diameter particle size-commonly referred to as lumps, are strongly self attenuating for gamma-rays typically used to determine radioisotopic information. Because of this, NDA techniques are susceptible to self-attenuation biases when the TRU material is in dense accumulations, agglomerations and/or large clumps. As an example, consider a sphere of $\mathrm{Pu}$ metal with a diameter, $D$. The fraction, $F$, of photons with energy, $E$, that can escape from such a sphere is given by

$$
F(D, E)=\frac{3}{X}\left\{1-\frac{2}{X^{2}}+\left[\frac{2}{X}+\frac{2}{X^{2}}\right] e^{X}\right\},
$$

where $X=\mu(E) \cdot D$ and $\mu(E)$ is the linear attenuation coefficient for the material at energy $E$.

As can be seen from this equation the escape fraction is strongly dependent on the absorption coefficient, which is a function of the photon energy. For $129 \mathrm{keV}$, less than $20 \%$ of such photons escape from a $1-\mathrm{mm}$ diameter sphere of $\mathrm{Pu}$, whereas almost $90 \%$ of the $414-\mathrm{keV}$ gamma rays would escape. This strong energy dependence may be able to be used to determine if there is a self-attenuation problem with the assay, and it can also be used to estimate the magnitude of this bias. The coarse size of the A\&PCT volume elements do not allow a direct observation and/or correction of this form of attenuation.

Our approach for correcting the A\&PCT assay data for self-attenuation will be as follows. We will reconstruct emission images for the 203-, 345- and 414$\mathrm{keV}$ gamma rays, and possibly the $129-\mathrm{keV}$ gamma ray. Then we will calculate their corresponding Pu masses. If there is no self-attenuation problem, these assay values will be equal. However, self-attenuation could be inferred if the assay results strongly increase with photon energy. A first order correction for this could be obtained by finding a best fit for the particle size that would 
reproduce the observed energy dependence. One could then recalculate the assay values using a correction for the escape fractions.

\section{II.B.3 Image Reconstruction and Assay Determination}

While it seems simple enough to assay the total radioactivity within a large distributed volume (a nuclear waste drum) by measuring the emitted radiation, the central difficulty is that an accurate absolute assay is impossible unless the emerging measured radiation can be corrected for the matrix attenuation it suffers. This correction requires knowledge of the spatial distribution and density of both emitters and absorbers throughout the volume. An accurate assay will necessarily involve a complete determination of the three-dimensional (3-D) structure of all radioisotopes present even though the original problem posed by the regulations requires only one number, the total radioactivity quoted as Pu-effective grams, contained within a waste drum.

The active CT data is acquired and reconstructed using the robust 2-D filtered backprojection method described elsewhere.[BUD79] The resultant 2-D ACT slice data at a particular energy is merged into a 3-D array before it is submitted with the passive count data to the passive $C T$ image reconstruction and assay algorithm.

In order to assay the 3-D structure of a waste drum, it is divided into a set of voxels. The number of counts for all detectable radioisotopes is determined for each voxel (see Eq II.A-E7). The sum of the counts from all of the voxels (see Eq. II.A-E9) determines the non-destructive assay of the drum. The passive CT image reconstruction and assay starts by defining a vector $\mathbf{p}$, where the elements in the vector represents the number of counts from each voxel. The vector $\mathbf{p}$ is the unknown and is the desired solution. The emitted radiation is measured at a series of detector positions, which constitute the ray sums in a passive CT scan. The, vector $\boldsymbol{g}_{\gamma}$ is defined (see Eq. II.A-E7), where each element in the vector is the measured radiation at a given detector position. The vector $g_{\gamma}$ is the sinogram data measured in the passive CT scan.

The relation between the vectors $\mathbf{p}$ and $\boldsymbol{g}_{\gamma}$ can be defined mathematically as

$$
g_{\gamma}=\mathbf{A p},
$$

The system matrix, A, represents and incorporates the effects of the system's geometry and the attenuation image determined from the active CT scan. $\mathbf{A}$ is given by

$$
\mathbf{A}(E, s, \theta)=\iint a(E, x, y, s, \theta) \delta(x \cos \theta+y \sin \theta-s) d x d y .
$$

The matrix $\mathbf{A}$ can be calculated. The matrix $\mathbf{A}$ is too large to be effectively inverted to solve for $\mathbf{p}$. Therefore, $\mathbf{p}$ must be determined from $g_{\gamma}$ and $\mathbf{A}$ using an iterative optimization technique. 


\section{II.B.3.1 Reconstruction Code Structure}

The iterative A\&PCT reconstruction codes are divided into an optimization and a model code. Figure II.B.3-1 shows a conceptual design of the reconstruction code. The optimizer consists of a cost function and a minimizer algorithm. The cost function calculates a scalar by comparison of the measured, $\boldsymbol{g}_{\gamma}$, to the calculated, $\hat{\boldsymbol{g}} \boldsymbol{g}$, passive sinogram. The minimizer section searches for the next best solution, $\hat{p}$. The model code determines the calculated passive sinogram from the current solution determined by the minimizer. The optimizer determines when the solution is acceptable, the final $\hat{p}$.

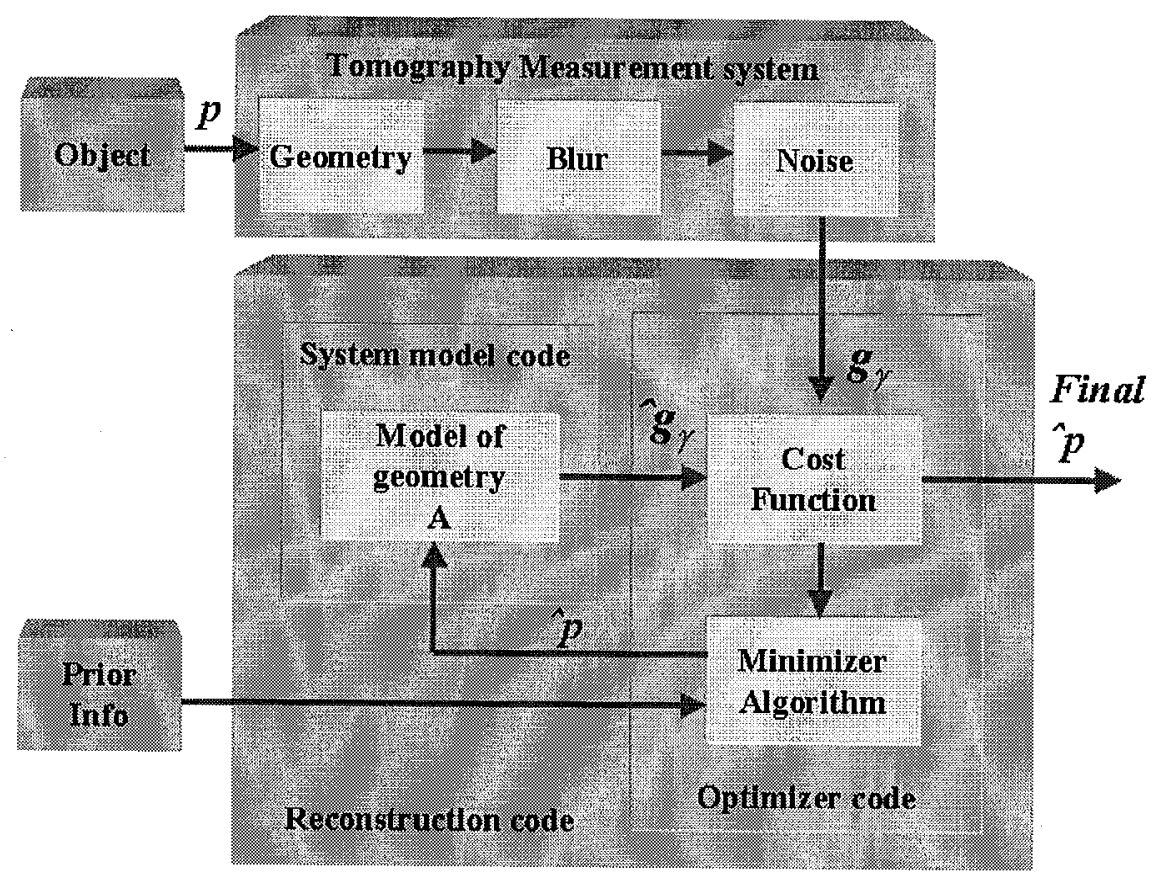

Figure II.B.3-1 Conceptual design of the passive image reconstruction code.

\section{II.B.3.1.1 Model Code}

The purpose of the model code is to calculate the values in the system matrix A. The values are determined by using the correct CT scanner geometry and physics to relate the contribution of the emission from each voxel to each detector. The effect of the measured attenuation from the active CT scan is also incorporated. In the recent past A\&PCT has used two methods to determine the system matrix, they are known as UCSF and APCT.

The first A\&PCT developed model code is called UCSF. There were several assumptions in the UCSF model code which were valid for the medical imaging case, but not for the drum imaging problem. A detector collimated to receive radiation from an emitting radioactive source measures photon counts 
decreased as the square of the distance between the emitter and the detector, $\left(1 / R^{2}\right)$, and also decreased exponentially by absorption along the line of sight. In addition, the collimation of high-energy photons is normally done with collimators rather than lenses, and the collimator aperture will have a diverging acceptance angle with a width or area proportional to distance from the source to the detector. Finite spatial resolution for both the active and passive measurements affects the resolving power of the system to measure the waste drum wall, waste items within the drum-including either emitters or absorbers with sharp boundaries-and the radioactive assay accuracy of all detectable emission sources.

The non-linear effects in the system, in particular the $1 / R^{2}$ fall-off, the exponential attenuation, and the spatially varying collimator response imply that reconstruction or inversion will not be a linear operation. Although emission tomography is not a new idea, most current applications do not fully account for these non-linear effects and do not provide for accurate quantitative measurements. In particular, in medical imaging, reconstructions are generally done with an inverse Radon transform. This implies the assumptions that the internal attenuation is not too strong, and that the distance through the object, the patient, is not too great so that the divergence of the collimators and the $1 / R^{2}$ fall-off are also small. In medical imaging it is possible to achieve a reconstruction of sufficient qualitative accuracy to be clinically useful in diagnosis. However, these effects cannot be ignored in quantitative imaging. The new model code, referred to as APCT, takes into account these issues. Also the attenuation line integral is calculated with finer sampling or spatial resolution than that used in the UCSF model code.

\section{II.B.3.1.2 Optimization Code}

Iterative methods for image reconstruction generally proceed by successive minimization or maximization of a cost function. For each situation a cost function must be determined and an iterative method for determining the maximum or minimum (optimization) of the function must be selected.

The cost function is usually a likelihood or log-likelihood function. In a system where a model exists for describing accurately the system, including non-linear effects, the likelihood function is often the squared difference between the model's predicted data and observed data. This is referred to as a least-squared likelihood function. Several numerical algorithms exist for least-squares minimization. Most of these algorithms proceed by moving down the gradient of the likelihood function. Examples of these algorithms are stcepest descent, conjugate directions, or successive projections. The last is termed the Algebraic Reconstruction Technique.

However, in the waste drum situation, where the system being modeled is the gamma rays produced by the decay of radioactive materials, the process is best represented by a Poisson probability distribution. This is shown where the probability on $n$ counts is $\operatorname{Pr}[n]$

$$
\operatorname{Pr}[n]=\frac{1}{n !} z^{n} \exp \{-z\} .
$$


The parameter $z$ is the mean number of counts. In our waste drum case, it is the measured gross or background counts at each detector ray-sum position.

An iterative reconstruction for determining the maximum likelihood for a Poisson distribution has been developed and used in the medical community.[SHE82] A version of this solution called Maximum Likelihood Expectation Maximization (MLEM) was developed in collaboration with UCSF for waste drum analysis. However, this MLEM method only handles one Poisson distribution signal. In our case there are two Poisson distribution signals, the gross and background counts. The MLEM algorithm is not flexible enough to incorporate these two Poisson distributed signals correctly.

We have developed a new likelihood function based on the joint probability density functions for the gross peak and the background for each ray sum measurement in a statistically correct fashion.[GOO97] This avoids any physically unrealistic "negative counts" that must be set to zero in other estimation approaches to this problem, such as MLEM. The result of zeroing negative counts can bias assay estimates (i.e., results). This new method avoids this problem. The likelihood function is minimized by a novel constrained conjugate gradient algorithm[GOO93] that permits constraints on the estimates (such as non-negativity) at each voxel and uses a bending linesearch technique to speed convergence.

Conventional techniques allow only a single variable per iteration to attain a bound, whereas the bending line search allows multiple variables per iteration to attain bounds. This search method is rapid and efficient. Given the dimensions of our problems of interest, this is a major advantage over the conventional techniques. This algorithm has been applied with great success to a variety of practical problems. These include deconvolution [GOO93], speckle interferometry [GOO93a], limited view x-ray tomography [KOL94, HAD95], and crystallography [SOM95] at LLNL, and pulsed photothermal radiometry [MIL95, MIL96, MIL96a, \& MIL96b] at the Beckman Laser Institute and Medical Clinic of the UC Irvine Medical School.

\section{II.B.3.2 Code Development History}

In order to determine the optimal method of image reconstruction and assay, our A\&PCT codes have undergone a series of improvements. We have progressed from parallel-beam through fan-beam to a cone-beam geometric methods to reconstruct the passive CT data. The parallel-beam geometry method used an iterative, steepest-descent, weighted least-squares technique [HUE77] to reconstruct PCT images with attenuation corrections from the ACT image.[MAR91a] The fan-beam or 2-D work is described by Roberson et al. in [ROB94]. Here, we only describe our development efforts for the 3-D methods. A schematic diagram of the 3-D development is summarized in Figure II.B.3-2, and each method is described below.

\section{II.B.3.2.1 UCSF-MLEM}

The first 3-D model code used in our A\&PCT studies was developed in collaboration with UCSF. It was adapted from a UCSF code specifically designed for medical imaging geometries, and we developed a 3-D model and 
an MLEM optimization method, which is referred to as UCSF-MLEM ${ }^{6}$. The line integral used to determine the attenuation is calculated on a voxel-byvoxel basis. The system matrix is essentially recalculated each time through the optimizer, in this case MLEM. It can also only use the MLEM optimization technique that it was originally built with. This code however does allow for the use of a measured response function. Details of this code are provided by Brown, et al.[BRO95, TAN98]

\section{II.B.3.2.2 APCT-MLEM}

In the APCT model code the system matrix is calculated once and stored. This makes the code more modular and allows other optimization techniques to be used with the same model code. Several combinations of the A\&PCT model codes with different optimization codes have been developed. These are APCT-MLEM; APCT-CCG; and APCT-CCG with measured response function. The APCT-MLEM code uses the new model code in conjunction. with the MLEM optimization code. The latter two codes are discussed in the following two sub-sections.
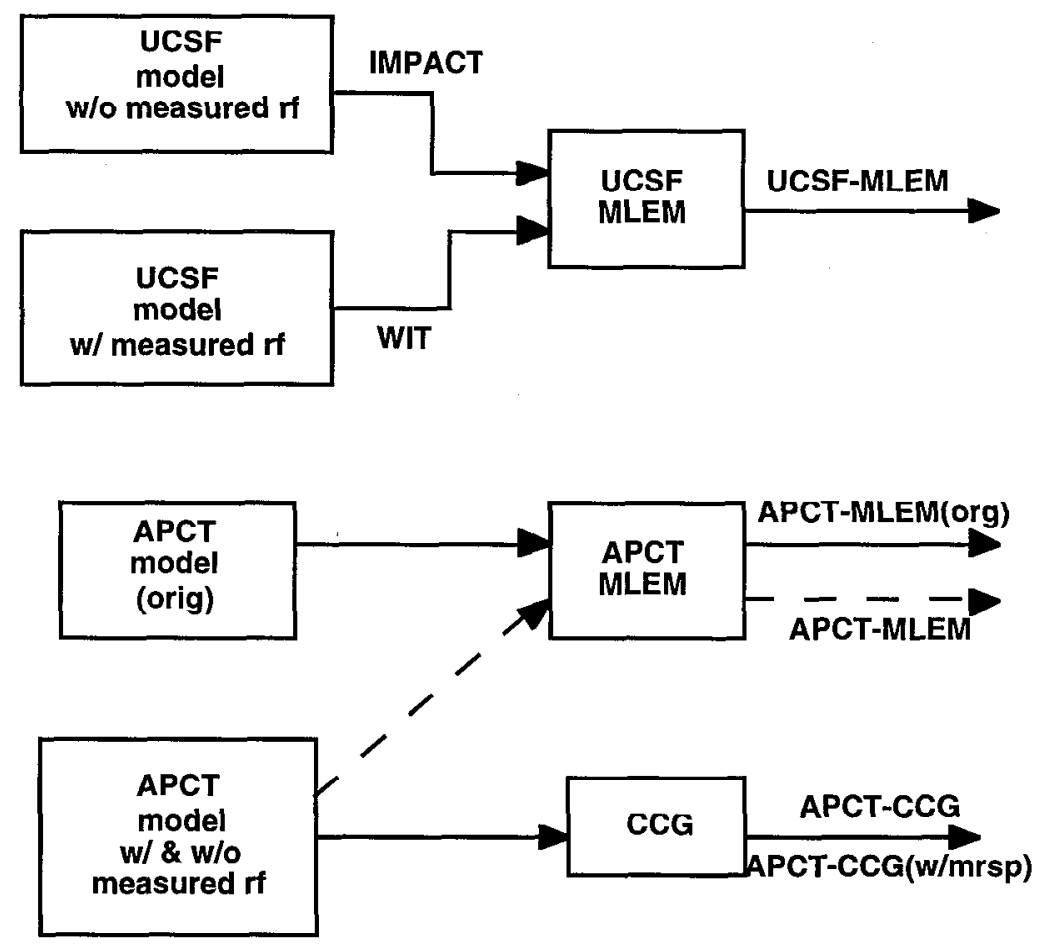

Figure II.B.3-2 A schematic diagram that summarizes the different $3 \mathrm{D}$ image reconstruction and assay codes developed. "rf" refers to the response function of the detector and its collimator.

\footnotetext{
${ }^{6}$ The nomenclature used in the text is to name the passive image reconstruction and assay codes by a hyphenation of the model and optimization code names.
} 


\section{II.B.3.2.3 APCT-CCG}

The APCT-CCG code is a combination of the APCT model code with the CCG optimization algorithm with a two measurement Poisson-based likelihood function. This code uses only a calculated response function.

\section{II.B.3.2.4 APCT-CCG with Measured Response Function}

The APCT-CCG with measured response function code is the same as the APCT-CCG code except it allows the user to choose to use a calculated or a measured point source (impulse) response function. This allows us to use one image reconstruction and assay code for both the WIT and IMPACT data.

\section{II.B.3.3 Results - Performance of Codes}

Two biases were noticed in the assay results when the UCSF-MLEM code was used to reconstruct and provide assay results for passive CT data. One bias is positive and seems to occur for poor statistical data. The other bias was negative and it was of the order of minus $30 \%$, and seemed to occur for good statistical data. Both biases are discussed in more detail in section III.B. The research work on the APCT model and CCG optimization codes was performed to reduce both biases.

To better understand the positive bias problem and to determine if the new codes help reduce this bias we have simulated several cases. Since the APCTMLEM and APCT-CCG codes use the same model code, the effectiveness of the optimization methods, MLEM and CCG, can be compared. The simulated sinogram data can be generated from a forward projection of a test image through the APCT model code. When the same model code is used in the forward projection and in the optimization, any differences in the results will be due to the effects of the optimization process.

The test cases were generated using a simulated 3-D image with three slices. Each slice was 14 by 14 voxels. A point radioactive emission source of selected counts was placed on the center slice at voxel location $(5,5)$, i.e., just off the center of the slice. Using the system matrix, A in equation II.B-E1, the image was forward projected to create three sinograms. A level background of a selected counts was then added to these sinograms. The simulated sinograms were randomized by passing them through a Poisson random generator. These sinograms represent the gross sinograms. Another set of sinograms were created with the same background level and randomized. These sinograms represent the background sinograms.

The gross signal consists of two components, the background and the net. The net is the contribution from the passive CT source. The desired result of the optimization process is the PCT image that produces the net signal. Although for Poisson distributions it is statistically incorrect to subtract background counts from gross counts to determine the net counts, for analysis purposes it is useful to determine the average gross counts per ray sum and the average background counts per ray sum and take the difference as the average net counts per ray sum. Sinograms from actual data sets were 
examined to determine the range of average counts per ray sum to be expected. Then a series of simulations were done to examine the effectiveness of the MLEM and CCG optimization algorithms under different conditions.

Three average background counts per ray sum were used; 1,5 and 20 . The simulated average net counts were achieved by selecting a value for the point emission source in the simulated image that yielded the desired average net counts. For each of the three average background values, six average net count values were used; 0.5, 1.0, 2.0, 3.0, 4.0 and 5.0; i.e., net-to-background values ranging from 0.025 to 5.0 . This allows us to examine the effect of low net counts on the optimization codes as well as the effect of the net-tobackground ratio. The APCT-MLEM and APCT-CCG simulation results are shown in Figure II.B.3-3. At least four observations can be drawn from our studies. For net counts greater than one, APCT-CCG performs $\sim 20 \%$ better than APCT-MLEM. APCT-CCG's performance degrades when net counts are too low $(\leq 1)$. The net-to-background ratio has almost no effect on the assay results for either optimization case, except for the 0.5 and 1 average net counts per ray sum APCT-MLEM cases. For these latter two cases APCT-MLEM fails.

The sinograms and images for the case of the average background of 20 counts and the average net of 2 counts are shown in Figure II.B.3-4. The APCT-MLEM image is spread out over 27 voxels (3-by-3 voxels within each slice and across all three slices). Its total assay yields 36710 counts, i.e., $21 \%$ higher than the actual value. The APCT-CCG results are more localized and its assay value of 30072 counts is much closer to the original 30350 counts, i.e., within $1 \%$. The other important observation is that the APCT-CCG code calculated sinograms are more representative of the original source or net sinogram data than the source plus background, i.e., gross data, which is not the case for the APCT-MLEM sinogram results. It is important to point out

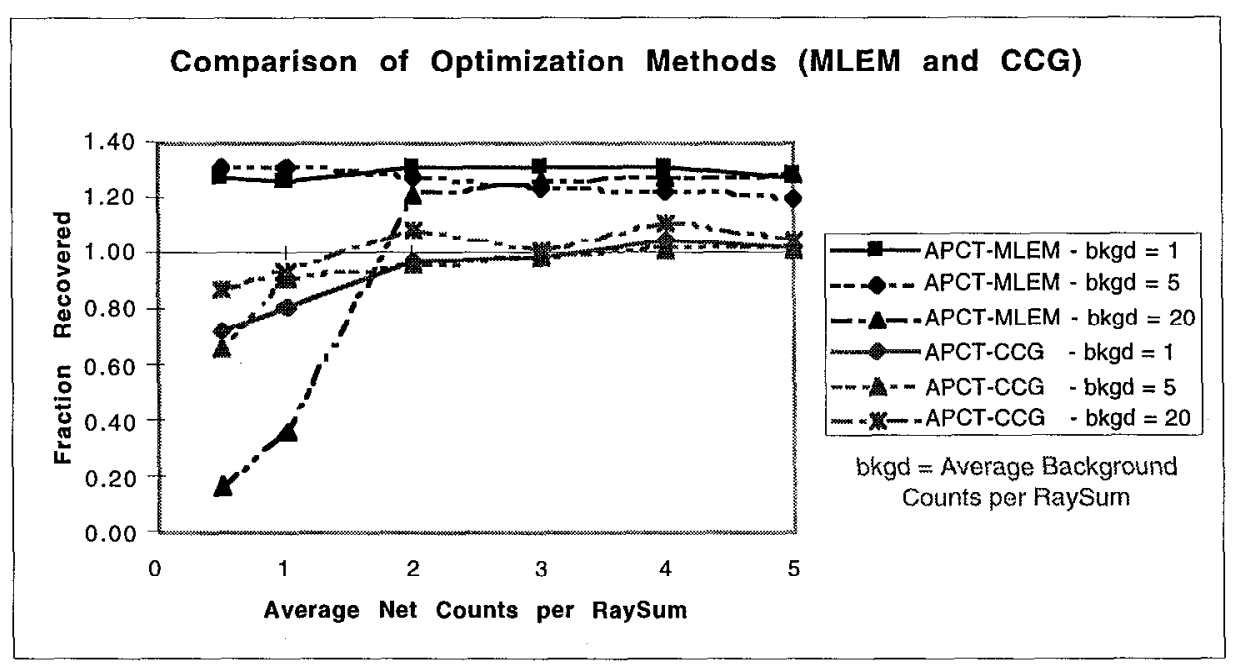

Figure II.B.3-3 Comparison of the MLEM and CCG optimization methods for different simulated data sets of net counts and background levels. 


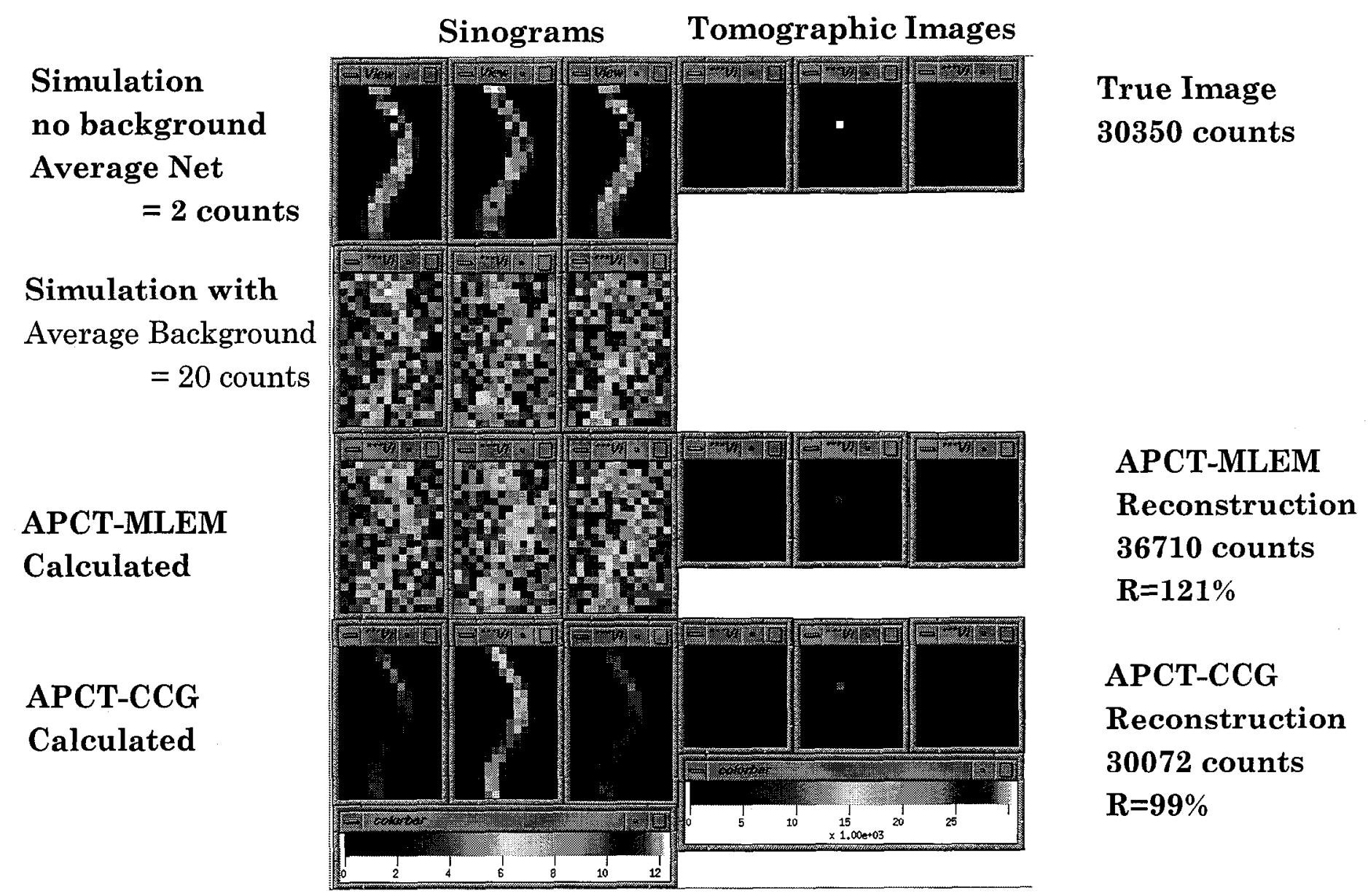

Figure II.B.3-4 Comparison of 3 sinogram images (left) and 3 CT slice images (right) for simulated data. After adding background (noise) to the simulated sinograms they were reconstructed into CT slices. The final assay result as given by the APCT-MLEM and APCT-CCG codes are shown. $R$ is the simulated- to true-mass value ratio in percent. 
that these simulated results are only useful for comparing the optimization algorithms. It does not shed any light on the effectiveness of the APCT model's accuracy in representing the waste drum A\&PCT assay problem.

The net-to-background simulation methodology just described cannot be quantitatively applied to real data and for that matter is only useful in learning about the optimization methods. We want to better understand both the algorithms model and optimization performance on real data. The average counts per ray sum method cannot be used on real data because they vary with the number of slices, the position of the emitter and the amount of attenuation within the drum. For real data we decided to plot the assay results as a function of ray sum integration time instead of average net counts per ray sum. The idea here is that as we decrease the integration time both the net and background count levels decrease.

We studied a PDP cycle 2 drum that contained $0.93 \mathrm{~g}$ of weapons grade (WG) $\mathrm{Pu}$ within an ethafoam matrix. This drum was scanned using several different ray-sum integration times. The average-net to average-background ratio is $\sim 1.8$ for all integration times acquired. The assay results as a function of net counts per ray sum are shown in Figure II.B.3.-5. For average net counts of $\sim 4$ the APCT-CCG algorithm assay shows a slightly positive recovery, while the UCSFand APCT-MLEM algorithms show good to incomplete recovery (negative bias). For average net counts of $<1$ assay recovery performance results for all codes are poor. The UCSF- and APCT-MLEM algorithms result in an increase in measured assays as a function of decreasing average net counts while the APCT-CCG algorithm shows a decrease. As in the simulation, APCT-CCG does not perform well for insufficient counts; however, it performs as good or better than the other codes when there are sufficient counts.

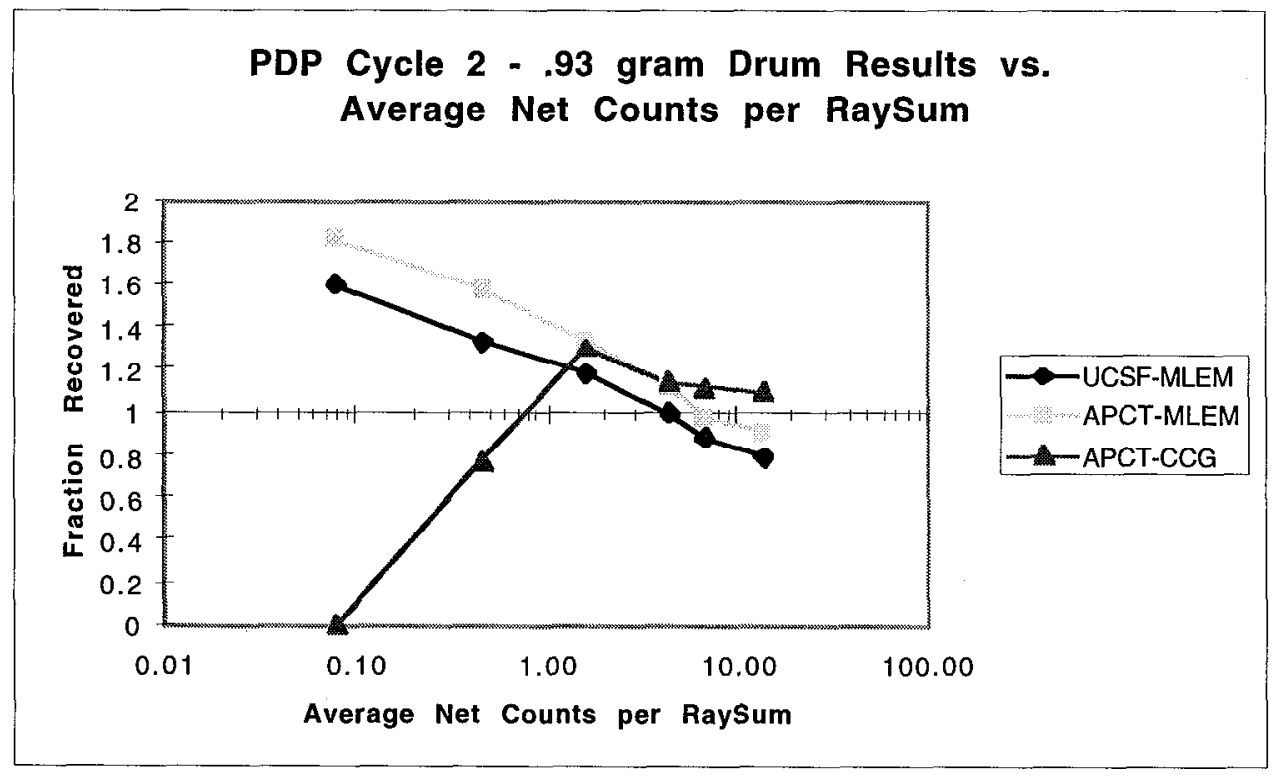

Figure II.B.3-5 Plot of fraction recovered versus ray-sum integration time for the ethafoam drum with 0.93 -grams of ${ }^{239} \mathrm{Pu}$ (PDP cycle 2 test). 
PDP cycle 2 passive sinograms and PCT images for two (17 and $160 \mathrm{~s})$ raysum integration times are shown in Figures II.B.3-6 and Figure II.B.3-7. As mentioned earlier the APCT-MLEM code cannot separate the net and background counts correctly, whereas the APCT-CCG code can solve for the magnitude of the signal itself. This may help explain why the APCT-CCG calculated sinogram, for both figures, compared to the APCT-MLEM calculated sinogram results seem to be more representative of the signal itself and not the signal plus background. In the 17-second case APCT-CCG appears to not yet have enough counts to perform well and its results are very similar to APCTMLEM. However, the sinogram for APCT-CCG appears to represent the actual measured sinogram much more accurately.

Finally, we used all the codes to determine and compare their assay performance for many data sets. Figure II.B.3-8 shows a comparison of the A\&PCT reconstruction and assay codes based on the fraction recovered, i.e., the ratio of calculated to true value for various experimental data. With respect to these figures the experimental data is as follows: (1) alpipe, basand, bana, and phantom are abbreviations for an $\sim 60 \mu \mathrm{Ci}{ }^{133} \mathrm{Ba}$ point source within an aluminum attenuating ring, sand, no attenuator, and a heterogeneous attenuator, respectively; (2) c15g20 and $\mathrm{o} 15 \mathrm{~g} 20$ are abbreviations for a 15-gram ${ }^{239} \mathrm{Pu} \mathrm{PDP}{ }^{7}$ standard source on the center-axis-of-rotation (c) and off the center axis (o), respectively, with no attenuators and 20-sec. ray-sum integration time; (3) pdp_160, 80, 50, 17, and 5.c2 represent the same cycle 2 PDP standards $(0.87$ grams of ${ }^{239} \mathrm{Pu}$ ) within an ethafoam matrix drum with ray-sum integration times of 160,80,50,17, and 5 seconds, respectively; (4) pdp003.c3 and pdp004.c3 represent the results for the cycle 3 PDP test with 71.36 grams of WG Pu $(66.87 \mathrm{~g}$ of $\left.{ }^{239} \mathrm{Pu}\right)$ within a combustible-matrix drum and 98.3 grams of WG $\mathrm{Pu}(92.11 \mathrm{~g}$ ${ }^{239} \mathrm{Pu}$ ) within a glass-matrix drum; and (5) pdp3.6g is a special test we performed using a combustible drum with 3.60 grams of WG Pu (3.37 grams of $\left.{ }^{239} \mathrm{Pu}\right)$. From these figures it can be seen that the APCT-MLEM code performs somewhat better than the UCSF-MLEM code. This difference reflects improvements in the model code. The APCT-CCG code performs, on average, better than both MLEM optimization codes. The difference between APCTMLEM and APCT-CCG reflects the effect of the optimization algorithm.

The fractions recovered are plotted versus the known ${ }^{239} \mathrm{Pu}$ mass values in Figure II.B.3-9. The data in Figure II.B.3-9 are for drums containing Pu with sufficient counts (good statistical results), which show a negative bias. The bias for all codes increases as the gram loading increases, though the percent change is greatest for APCT-CCG. If we regard all of the data shown in Figure II.B.3-9 as valid, the mean negative biases are: $-26 \%$ for UCSF-MLEM; $-18 \%$ for APCT-MLEM; and $-7 \%$ for APCT-CCG. Thus, APCT-CCG seems to perform consistently better than the other two.

\footnotetext{
${ }^{7}$ Performance Demonstaration Program
} 


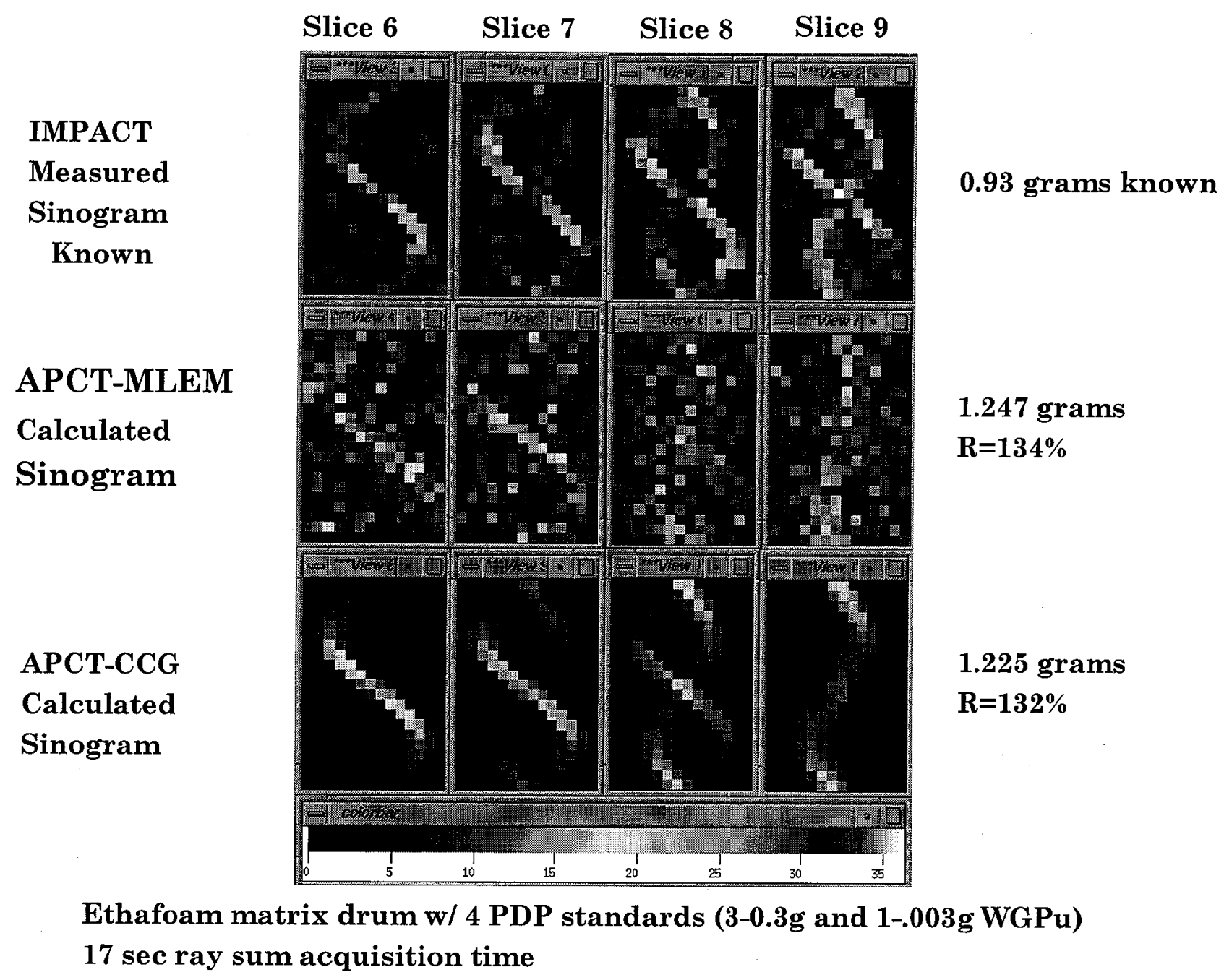

Figure II.B.3-6 Comparison of measured (acquisition time of $17 \mathrm{sec} . /$ ray sum) and calculated sinograms for four slices of the empirical PDP cycle 2 ethafoam drum. $R$ is the measured- to true-mass value ratio in percent. 


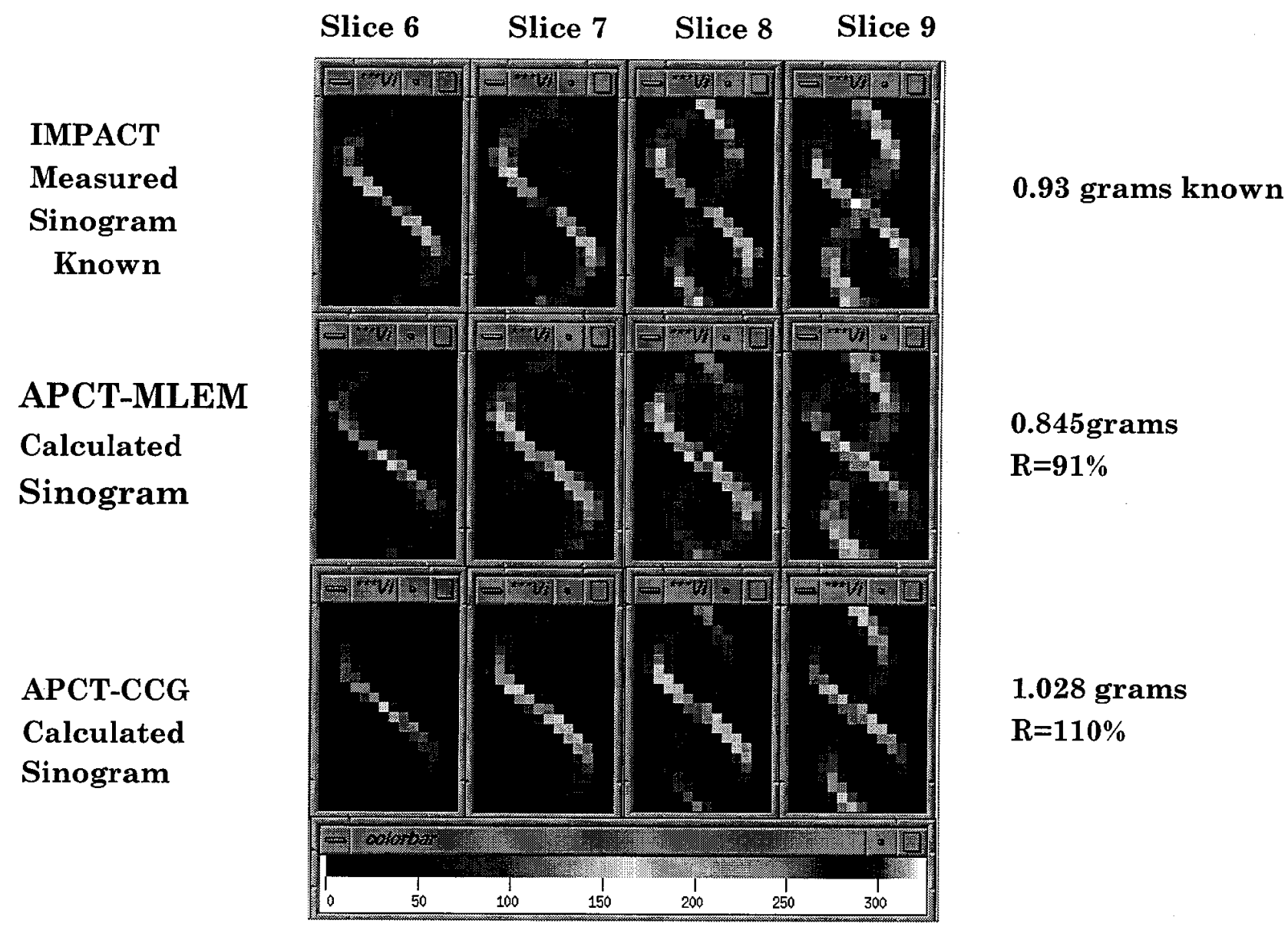

Ethafoam matrix drum w/ 4 PDP standards (3-0.3g and 1-.003g WGPu) 160 sec ray sum acquisition time

Figure II.B.3-7 Comparison of measured (acquisition time of $160 \mathrm{sec}$./ray sum) and calculated sinograms for four slices of the empirical PDP cycle 2 ethafoam drum. $R$ is the measured- to true-mass value ratio in percent. 


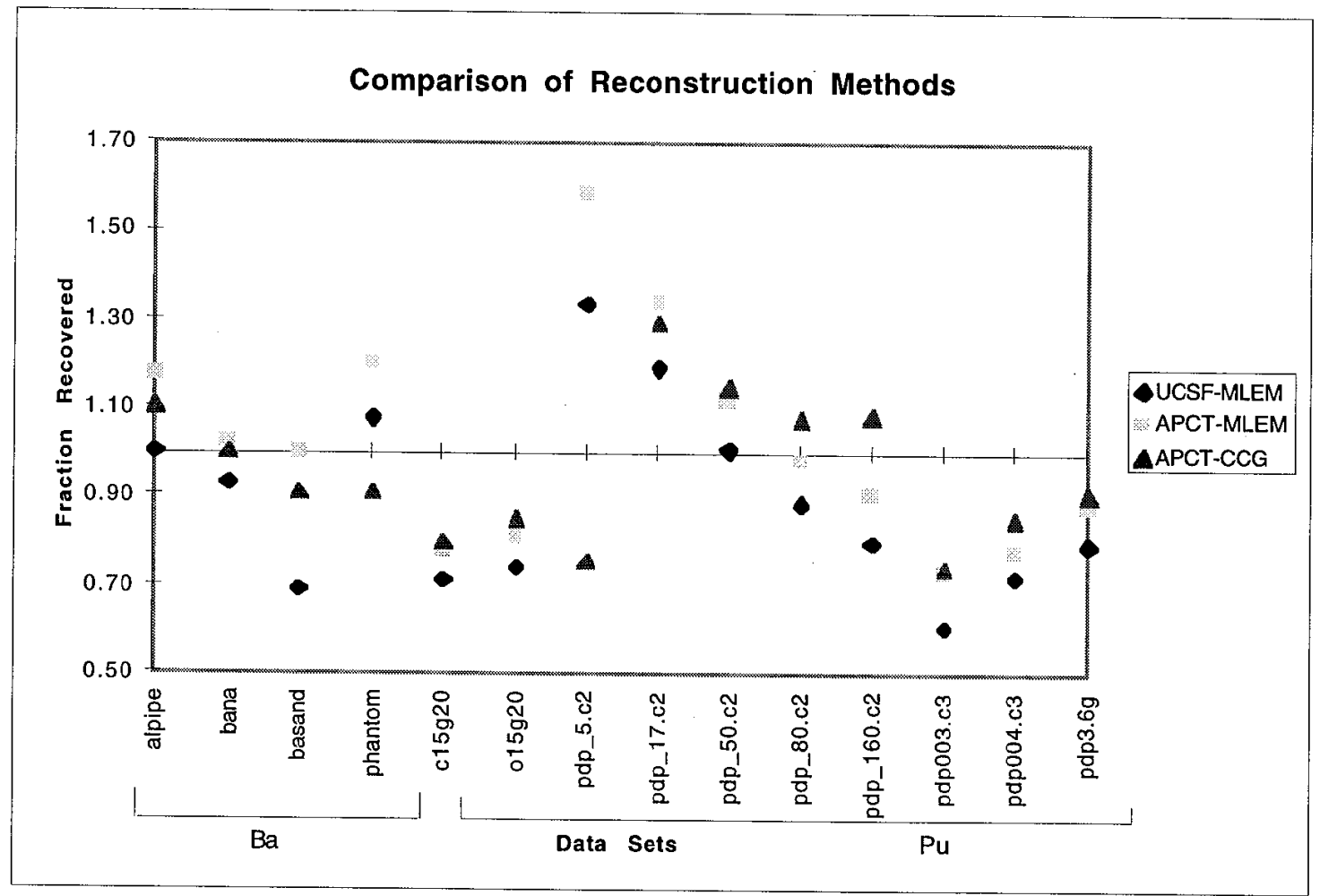

Figure II.B.3-8 Comparison of reconstruction methods for several empirical samples with known assay values.

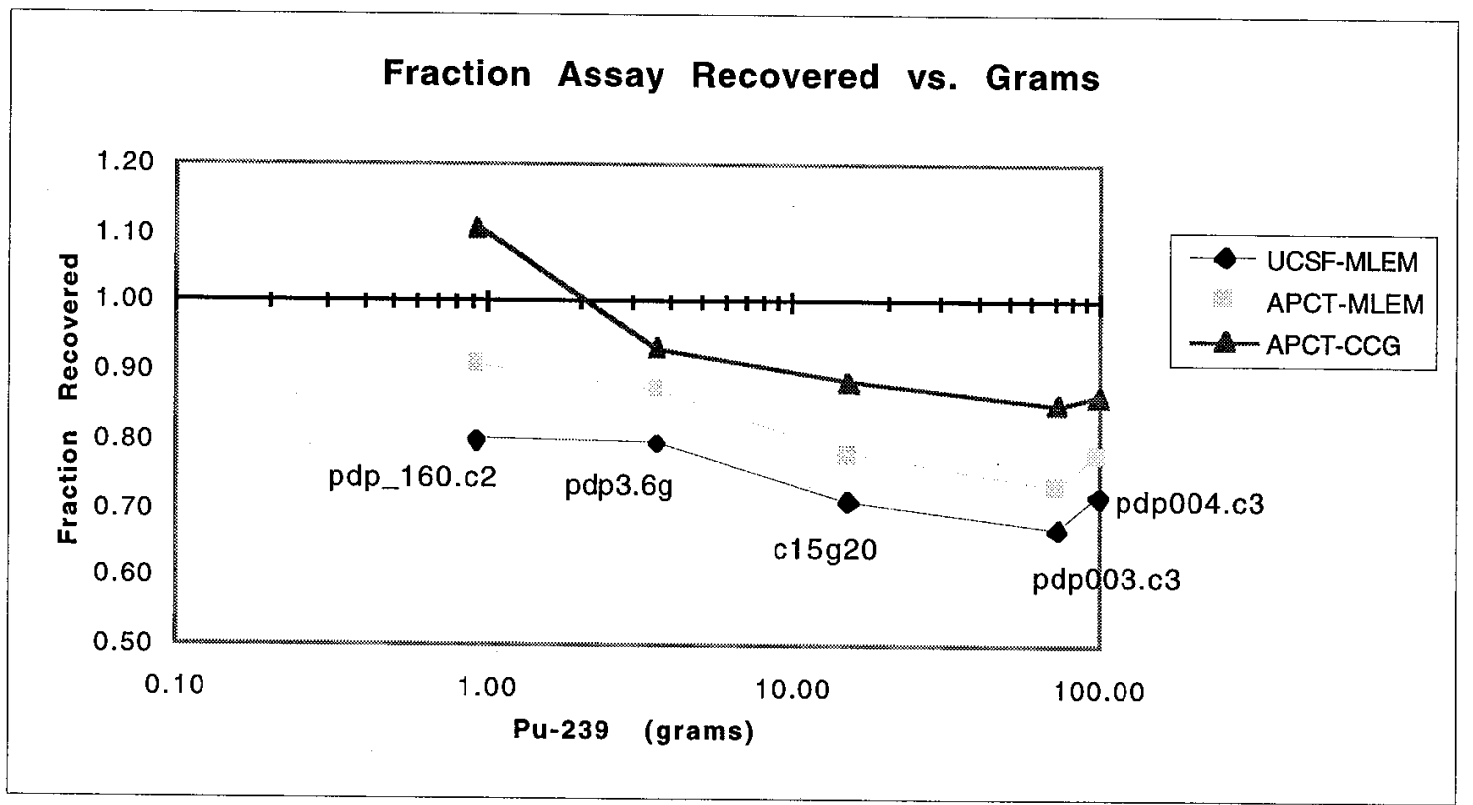

Figure II.B.3-9 Plot of ${ }^{239} \mathrm{Pu}$ fraction recovered vs. increasing ${ }^{239} \mathrm{Pu}$ gram amounts for several test drums as labeled. 


\section{II.C Operational Configuration}

Over the past decade, LLNL has developed four A\&PCT systems to research and develop this technology. The first was a one-sixth scale system using a large aspect ratio (60:1). This earlier system demonstrated clearly that one can use mono-energetic ACT to image the matrix attenuation and that this properly corrects the passive emission CT data to yield an accurate measure of the internal emission sources.[MAR92] The second system was a full-scale 208-liter scanner constructed at LLNL's Site 300. This system was instrumental in the development of protocols for the calibration, validation, simulation, testing and demonstration of the A\&PCT technology.[ROB94]

Over the past five years we have developed two additional A\&PCT systems to demonstrate this technology on real waste drums. These are LLNL's IMPACT (Isotope Measurements by Passive and Active Computed Tomography); and BIR's WIT/A\&PCT (Waste Inspection Tomography/Active and Passive Computed Tomography).

The IMPACT system is located at LLNL and its primary purpose is to further research and develop the A\&PCT technology and to demonstrate and verify its concepts. It was not developed to assay real waste on a production basis; however, we have assayed a few real waste drums for demonstration purposes.[ROB95] Also, IMPACT officially participated in the third cycle of the National TRU Program sponsored Performance Demonstration Program (PDP).

The WIT/A\&PCT project is a collaborative effort between LLNL and BIR to integrate the A\&PCT technology into a mobile trailer. The WIT system was developed to perform demonstrations of the A\&PCT technology and to become a certified production-mode nondestructive assay system. The system has traveled to several different DOE facilities (e.g., LLNL, RFETS ${ }^{8}$, INEEL, and $\mathrm{NTS}^{9}$ ) to perform demonstrations on known and real waste drums. The WIT system has participated in three formal DOE tests: (1) The fourth cycle of the PDP; (2) A Rapid Commercialization Initiative (RCI); and (3) The Capability Evaluation Project (CEP). All of these test were conducted at INEEL.

\section{II.C.1 IMPACT Configuration}

\section{II.C.1.1 Hardware}

A photograph and schematic of the IMPACT scanner is shown in Figure II.C.1-1. It consists of two towers of interlocking space-frame aluminum tubing. One tower supports a $2.8-\mathrm{mCi}{ }^{166 \mathrm{~m}} \mathrm{Ho}$ (1200-yr. half-life) radioactive gamma-ray source that is used for the active CT mode.[ROB94] The active source has a manual shutter and a collimator with a 25.4-mm square aperture and $50.8-\mathrm{mm}$ length. The ${ }^{166 \mathrm{~m}}$ Ho source produces gamma rays at $184-, 280-$, 365-, 411-, 530-, 712-, and 810-keV; and provides crucial, energy-specific, attenuation information for a range of items that reside within the waste

\footnotetext{
${ }^{8}$ Rocky Flats Environmental Technology Site

${ }^{9}$ Nevada Test Site
} 

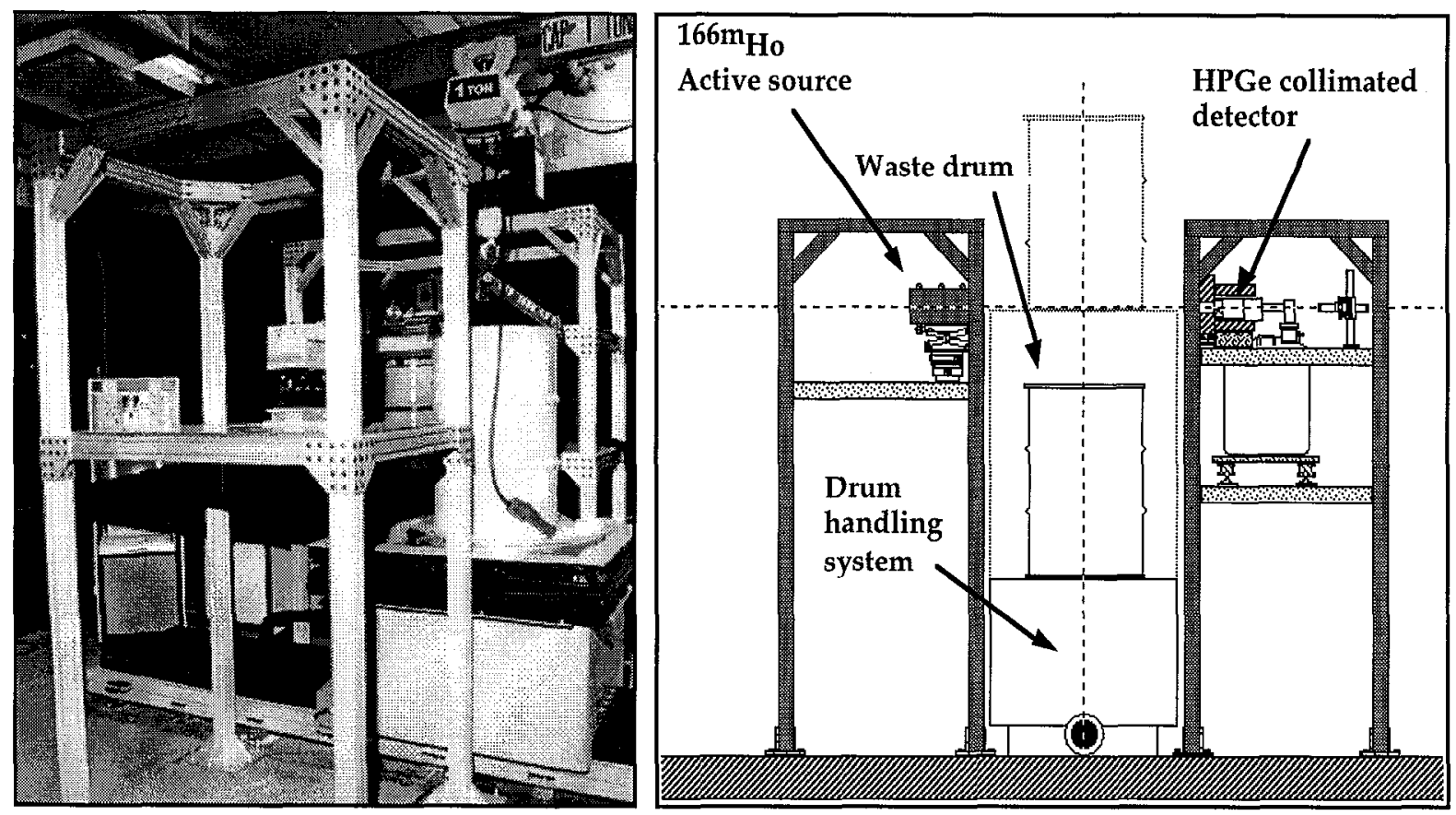

Figure II.C.1-1: A photograph (left) and schematic (right) of the IMPACT scanner.

matrix. We have found that it is best if the active-source peaks are not to close in energy to any passive-source gamma-ray peaks emitted from within a waste container. If the active peaks are too close in energy it can interfere with the passive measurement and thus affect the final assay result. It was shown experimentally that interpolation between any two nearest neighboring active peaks (529.8 and $280.5 \mathrm{keV}$ in ${ }^{166 \mathrm{~m}} \mathrm{Ho}$ ) resulted in reconstructing the same mass for the $414-\mathrm{keV}$ peak of ${ }^{239} \mathrm{Pu}$ as using the $411-\mathrm{keV}$ active peak data of ${ }^{166 \mathrm{~m}} \mathrm{Ho}$.

The other tower supports a single, well-collimated, $90 \%$ efficient (relative to a $7.6 \mathrm{~cm} \times 7.6 \mathrm{~cm}$ ( 3 in. $\times 3$ in.) NaI(Tl) detector at $1.33 \mathrm{MeV}$ ) HPGe detector. The detector has a collimator with an aperture size of $50.8 \mathrm{~mm}$ in the horizontal and vertical dimensions and a length of $254 \mathrm{~mm}$ (an aspect ratio of 5:1). We have verified through experiments that this aspect ratio provides an accurate assay.[DEC96]

The towers are designed to be versatile so that additional sources and detectors could be added to the scanner in the future. Between the two supporting towers is a three-axis staging system for drum manipulation. The staging system is capable of translating and elevating a $450-\mathrm{kg}$ (1000-pound) waste drum $125 \mathrm{~cm}$ (50 inches). In addition, the stage is capable of continuous or step wise rotation of the drum through $360^{\circ}$. It should be noted that IMPACT has a platform that elevates the drum above the rotation table to allow one CT slice to be acquired below the waste drum. This provides a better measurement of any radioactive content that may lie on the bottom of the drum since it accounts for the acceptance angle of the detector aperture. The drum manipulator and towers were designed to be robust with safety features necessary for handling TRU waste in a seismical active area. Engineering 
safety notes were developed to cover all operations of scanning for quality and safety controls.

A one-ton jib crane is located at one end of the scanner and provides easy and safe manual loading of the heavy drums. The crane must be interlocked in a home position away from the drum manipulator to insure that a waste drum cannot be driven into the jib crane during system operation.

\section{II.C.1.2 Data Acquisition}

IMPACT uses a PC for system control and data acquisition. During both active and passive operations, the PC discretely positions the drum for data acquisition. After positioning, it communicates with a multi-channel analyzer (MCA) that acquires data from the HPGe energy-discriminating detector. The detector integration time is a preset variable and can be adjusted depending on the amount of activity within the drum and the type of attenuating waste matrix. When the counting integration time is completed, the control computer downloads the MCA's data and stores selected energy regions of interest and/or the entire spectrum onto a system disk. After data storage, the drum is moved to the next position and the next integration is obtained.

IMPACT includes both the CGS and A\&PCT protocols. The CGS protocol is discussed in Section II.B.1.3. For the active CT mode, IMPACT translates the drum in 50.8- $\mathrm{mm}$ increments for each ray sum after the specified ray-sum integration time. A 208- $L$ drum is translated 14 times over a distance of 711.2 $\mathrm{mm}$ and then rotated approximately $8.5^{\circ}$ (21 rotations over $\left.180^{\circ}\right)$. Each set of 14 ray sums make up a projection. After each rotation, the next projection is acquired. After completion of all 21 projections the drum is elevated $50.8 \mathrm{~mm}$ and 21 new projections are measured for the next slice plane. The drum is elevated 18 times to assay a completely full 208-liter drum; fewer elevations are required if the drum is not full.

The drum is correctly sampled in the passive mode with fewer ray sums and projections because of the large acceptance angle of the detector's collimator aperture. Its effective-acceptance angle is larger in the passive mode than in the active mode. Thus, for passive mode data collection, the drum is translated to obtain only 7 ray sums over a distance of $711.2 \mathrm{~mm}$ (101.6-mm increments). Next, the drum is rotated $36^{\circ}$ since only 10 projections are required over $360^{\circ}$ for each slice. Once again, the PCT protocol requires 18 slice planes to image a completely full 208-liter drum.

The data acquired from IMPACT is processed and analyzed on a UNIX work station. A UNIX file system is mounted by the PC and data are transferred over an ethernet cable. Both the energy regions of interest (EROI's) and/or spectra that represent each individual data measurement are transferred.

The EROI data is simply the integrated photon counts within an energy peak of interest for some specified integration time. The EROI is set prior to the assay on the known energy peaks of the active or emission sources. The 
EROI data is processed and reconstructed without any need for further isotopic analysis, since we mainly focused on WG Pu during the research and development phases of this work. The disadvantage of using the PCT EROI data for the A\&PCT reconstruction is that the type of emission sources that are being evaluated within the drum must be known prior to the assay. This may not be the case for all waste drums being assayed; therefore, there is an additional option to save the spectra acquired for each PCT ray sum. As previously mentioned, this option has been used to determine from PCT data the TRU isotopics ratios in a waste drum prior to image reconstruction and final assay.

\section{II.C.1.3 Data Reduction}

All performance data shown is reduced using the UCSF-MLEM image reconstruction and assay code. The performance data acquired and shown for IMPACT was obtained over several years. We did not use the newly developed APCT-MLEM or APCT-CCG codes since they were not available until just recently. The APCT-CCG code shows a reduction of about three in our negative bias as shown in Figure II.B.3-9. Since we did not have the time to run the WIT/APCT data using the new codes, we show only the IMPACT UCSF-MLEM performance results so that we can compare them to the WIT/APCT scanner UCSF-MLEM performance results.

\section{II.C.1.4 Calibration of IMPACT}

Our goal has been to design a gamma-ray waste drum nondestructive assay technology that does not require special calibration as a function of the type or distribution of waste matrix, or for the type or distribution of gammaray radioactive sources within a drum. LLNL's A\&PCT technology does not require prior knowledge of, or calibration for, the waste stream that is being assayed.

For the PDP cycles, we could have calibrated the A\&PCT scanners using drum matrices and source structures that are similar to the PDP drums and we may have attained assay results that would have been much more accurate. However, if we were to have calibrated to such a specific test or a specific waste stream, then the drums used for calibration would have had to have exactly the same as all the other drums scanned under that particular calibration. This is not the case for real waste stream drums, i.e., they can differ greatly from drum to drum for the same waste stream. That is why we consider it important to assay waste matrices and source structures of all kinds and there by remove the dependency on calibration procedures. It has been our conviction that the A\&PCT technology is an ideal technique for any waste stream; and it is especially valuable for waste drums whose contents are unknown or are suspected of containing something other than what is documented on their manifests. The A\&PCT technology requires only an understanding of the physics of the measurement process and the value of the absolute efficiency of the HPGe detector as a function of energy to provide an accurate quantitative nondestructive assay measurement for all waste drums independent of their waste content. 
To obtain an absolute assay measurement, the A\&PCT systems are calibrated once on an absolute detector-efficiency scale by simple measurements of a calibrated radioactive point source. We can do this because the computed tomography method takes into account the geometry of the source and detector, and their collimators. We do not need additional calibrations for different $\mathrm{Pu}$ gram-amounts or for different types of attenuating waste matrices, since they are measured in the A\&PCT gammaray method of nondestructive assay.

For the IMPACT scanner at LLNL our large-volume HPGe detector has a diameter of $7.6 \mathrm{~cm}$ (3-in.); the collimator aperture is a 5.08 by $5.08-\mathrm{cm}$ square and is $25.4-\mathrm{cm}$ long. This means that any unscattered gamma rays accepted by the collimator aperture will strike a reasonable volume of germanium, i.e., all unscattered gamma-rays strike the detector. Furthermore, because the collimator is $25.4-\mathrm{cm}$ long the incident angles of these photons are close to being normal to the detector surface. This makes the probability for the detection of a gamma-ray by the detector approximately proportional to the collimator acceptance.

The reconstruction code accounts for the collimator acceptance during image reconstruction and assay. The simple geometry of our system makes this rather straightforward. The proportionality factor can be determined by measuring a calibrated radioactive point source on the axis of the collimator at various distances. For this geometry we can calculate the solid angle of the collimator and compare it to the measured efficiency. For gamma rays at 400 $\mathrm{keV}$ about 0.5 result in full-energy peak events; and since we use a source that emits multiple, mono-energetic gamma-rays, we calibrate for all emission gamma-ray energies of interest. This calibration procedure is performed once prior to system operation and only takes a few hours to complete.

In either the IMPACT or WIT systems the calibration need be done only once since it will change only if the detector collimator is changed or if a detector problem develops. The detector's performance is easily verified by examination of the active data in each run.

\section{II.C.1.5 Validation of IMPACT}

In order to validate the IMPACT system we perform measurements on several known test cases. These cases range from simple tests such as well characterized radioactive point sources-without attenuators, within uniform attenuators, or simple heterogeneous attenuators to more difficult tests such as distributed sources within mock-waste drums of heterogeneous or very dense "sludge" matrices. Validation was accomplished through an analysis of the simple test cases and experiments on these mock-waste drums containing well-calibrated radioactive sources. The validation process also included characterization of WIPP PDP drums and sources when they became available.

We performed a series of experiments to demonstrate the performance of the A\&PCT method for characterizing radioactive waste drums. Our first experiments focused on the ability to correct passively measured data with an 
ACT (active) attenuation map. These experiments include a reference PCT scan acquired with only a passive CT source, i.e. no drum or waste items were present; hence, there was no attenuation of the source. Then, passive CT measurements were taken using different attenuators including a 208- $L$ drum filled with one or more mock-waste matrices and their attenuation corrected results were compared to the bare-source PCT reference scans. Thus, the success of our preliminary measurements was not the agreement of an absolute assay with the known activity of the source(s); but rather, how well we were able to correct for attenuation by the drum and its various mockwaste matrices.

Additional sets of measurements were obtained to study the A\&PCT reconstruction algorithm(s) and their ability to perform an absolute measurement. These measurements were performed with calibrated passive sources (e.g. ${ }^{133} \mathrm{Ba}$ ) and no waste matrix (corrected with null matrix); simple uniform attenuators (e.g. hollow aluminum cylinders or ring and a carboy of sand); or mock-waste drums with complicated waste matrices. Measurements acquired using the PDP drums provide a final validation of the A\&PCT technique.

The validation/baseline studies include the following measurements:

- Empty drum-different waste matrix drums without emission sources used to determine background and minimum detectable concentrations (MDCs);

- Point source with no attenuators-simple assay check;

- Point source with a uniform Al annular ring attenuator-assay check with uniform, low attenuation;

- Point source with a uniform sand matrix-assay check with uniform, high attenuation.

- Point source with a heterogeneous (sand, paper, and Al) attenuator-rotational verification and assay check with non-uniform attenuation.

All of these measurements were used to check/verify the data acquisition hardware and software, the gamma-ray spectral analysis, and the image reconstruction for nondestructive assay determinations. Once these were shown to be correct, we investigated A\&PCT assays of mock-waste drums. In general, our approach was to use simpler mock-waste drums with known radioactive sources to carry out initial A\&PCT scans, then move to the use of "calibration" drums used to calibrate LLNL's SGS system, and finally, use PDP drums when they became available.

Once completed, none of the above experiments have to be repeated unless the IMPACT system hardware or software goes through a major change, or unless a different data acquisition protocol is chosen or required. Changes to the gamma-ray spectral analysis software or image reconstruction software do not require that any data from the above experiments be reacquired. The existing data is simply rerun through any new software 
developed. Once these experiments were shown to be successful; and we had run a sufficient number of experiments to address the WIPP WAC criteria, then we were ready to assay real-waste drums.

A $63.6-\mu \mathrm{Ci}{ }^{133} \mathrm{Ba}$ point source was assayed to verify the operation of the IMPACT scanner and our reconstruction codes. The 356-keV peak was used in the assay. The point source was placed approximately 12 inches off the axis of rotation and was scanned with no attenuation. Five slice planes were acquired centered about the barium source so that a 3-D image could be reconstructed where all the activity is included. Figure II.C.1-2 shows three of the five slice images that represent the reconstructed central slices of the ${ }^{133} \mathrm{Ba}$ point source. The location of the point source is well defined in the PCT images. The UCSF-MLEM determined activity is in good agreement with the known value to within $6 \%$ as shown in Table II.C.1-1.

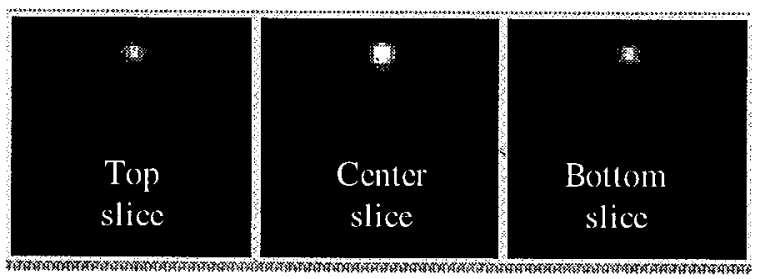

Figure II.C.1-2: Three of five PCT reconstructed images using UCSF-MLEM at $356 \mathrm{keV}$ for a $63.6-\mathrm{Ci}^{133} \mathrm{Ba}$ radioactive point source.

Table II.C.1-1: Assay of $\boldsymbol{a}^{133}$ Ba emission source
\begin{tabular}{|l|c|}
\hline 63.6 $\mu \mathrm{Ci}$ & 3-D UCSF-MLEM code \\
\hline Total Counts (measured) & $3.86 \times 10^{6}$ \\
\hline Activity (measured) & $67.3 \mu \mathrm{Ci}$ \\
\hline$\%$ Recovery & $105.5 \%$ \\
\hline
\end{tabular}

A comparison of the different A\&PCT image reconstruction and assay codes for several test cases is provided in Table II.C.1-2 and Figure II.B.3-8. All the data agree to better than 19,34 and $31 \%$ of the known value for the UCSFMLEM, APCT-MLEM and APCT-CCG codes, respectively, except for the ethafoam ( $5 \mathrm{sec}$.) case. For the ethafoam $(5 \mathrm{~s})$ case the APCT-CCG code dramatically reduces the positive bias shown in the MLEM optimization codes. Lastly for the 15-gram WG Pu PDP standard with no attenuator the APCT-CCG code provides the best recovery, $\sim 90 \%$, of the true source mass. 
Table II.C.1-2 Comparison of the different A\&PCT image reconstruction and assay codes performance for known tests with a ${ }^{133} \mathrm{Ba}$ point source, PDP standards within an ethafoam matrix drum and a PDP standard with no attenuators.

\begin{tabular}{|l|c|c|c|c|c|c|c|}
\hline \multirow{2}{*}{$\begin{array}{c}\text { Sample } \\
\text { Source/Attenuator }\end{array}$} & \multirow{2}{*}{$\begin{array}{c}\text { True } \\
\text { Value }\end{array}$} & \multicolumn{2}{c|}{ UCSF-MLEM } & \multicolumn{2}{c|}{ APCT-MLEM } & \multicolumn{2}{c|}{ APCT-CCG } \\
\cline { 5 - 9 } & Assay & $\% \mathbf{R}$ & Assay & $\% \mathbf{R}$ & Assay & $\% \mathbf{R}$ \\
\hline${ }^{133} \mathrm{Ba} /$ no-atten. & $57^{1} \mu \mathrm{Ci}$ & 53.1 & 0.93 & 58.1 & 1.02 & 59.9 & 1.05 \\
\hline${ }^{133} \mathrm{Ba} / \mathrm{Al}$ pipe & $57^{1} \mu \mathrm{Ci}$ & 57.1 & 1.00 & 62.8 & 1.10 & 60.8 & 1.07 \\
\hline${ }^{133} \mathrm{Ba} /$ Sand & $57^{1} \mu \mathrm{Ci}$ & 39.4 & 0.70 & 56.9 & 1.00 & 56.6 & 0.99 \\
\hline${ }^{133} \mathrm{Ba} /$ Heterogeneous & $57^{1} \mu \mathrm{Ci}$ & 61.4 & 1.08 & 68.5 & 1.20 & 54.6 & 0.96 \\
\hline \hline WG Pu/Ethafoam (17 sec.) & $0.93 \mathrm{~g}$ & 1.11 & 1.19 & 1.25 & 1.34 & 1.22 & 1.31 \\
\hline WG Pu/ Ethafoam (5 sec.) & $0.93 \mathrm{~g}$ & 1.30 & 1.40 & 1.47 & 1.58 & 0.73 & 0.78 \\
\hline \hline WG Pu/ no-atten. C ${ }^{2}(20 \mathrm{sec})$. & $15.0 \mathrm{~g}$ & 10.7 & 0.71 & 11.7 & 0.78 & 13.2 & 0.88 \\
\hline WG Pu/ no-atten. O ${ }^{3}(20 \mathrm{sec})$. & $15.0 \mathrm{~g}$ & 11.1 & 0.74 & 12.2 & 0.81 & 13.2 & 0.88 \\
\hline
\end{tabular}

Notes: 1. At the time of the assay the ${ }^{133} \mathrm{Ba}$ source had decayed to an activity of $57 \mu \mathrm{Ci}$.

2. Center, i.e., the source was positioned on the center-axis-of-rotation of the rotational table.

3. Outside, i.e., the source was positioned about 1 foot from the center-axis-of-rotation of the rotational table. 


\section{II.C.2 WIT/A\&PCT Configuration}

\section{II.C.2.1 Hardware}

We have performed work as a subcontractor to Bio-Imaging Research Inc., to integrate our A\&PCT technology into a mobile trailer that has two additional nondestructive evaluation (NDE) technologies. These three NDE/NDA technologies provide a complete $x$ - and gamma-ray waste inspection tomography (WIT) capability.[BER95, BER97, MAR95] They permit inspcction of waste drums to be carried out at many waste-drum storage sites. These technologies help characterize waste drums up to 416-liters (110-gals.) with weights up to $725 \mathrm{~kg}$ (1600 lb.); and containers up to 92-cm (3 ft.) diameter and 122-cm (4-ft.) tall. Figure II.C.2-1 shows a photograph of the semi-trailer (top) and a schematic layout of the equipment (bottom) within the trailer. The 60-ft.-long by 8.5-ft.-wide trailer is divided into four areas. The rear area provides space for two 160- $L$ liquid nitrogen containers, calibration and phantom drums, a 60k BTU heating and air-conditioning unit, and
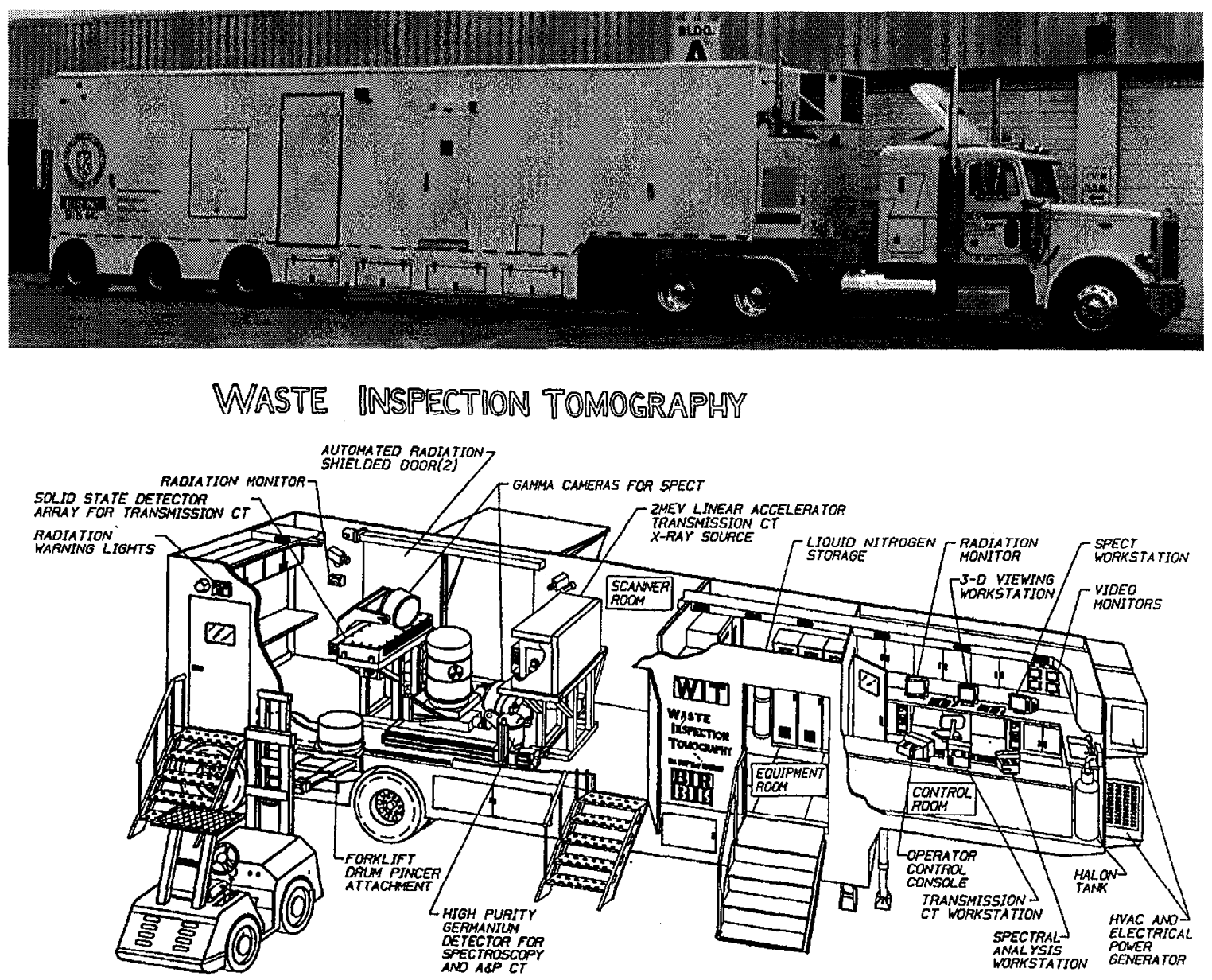

Figure II.C.2-1: A photograph of BIR's mobile waste inspection trailer and schematic of the equipment layout. 
storage lockers. The next area is dedicated to drum inspection and contains a 2-MeV linear accelerator for transmission computed tomography (TCT) and/or digital radiography (DR). This accelerator is supported by 896 cadmium-tungstate, solid-state detectors mounted in an array on individual photodiodes with septa between each detector to minimize cross talk, inplane scatter and blooming. To measure emitted gamma-rays, there are two large, collimated, sodium-iodide [NaI(Tl)] detectors similar to Anger cameras used in nuclear medicine for single photon emission CT or SPECT. To identify gamma-ray isotopics there is a highly collimated HPGe detector. A collimated $1.4-\mathrm{mCi} 166 \mathrm{~m}$ Ho radioactive source is used to obtain energy specific attenuation data in the active mode. This is not as strong an active source as could have been used. The third area is an equipment room and entry/exit to the trailer. The most forward area contains a control room and all of the supporting electronics where waste inspection personnel use several computer terminals to operate the several NDE/NDA measurement technologies.

\section{II.C.2.2 Data Acquisition}

The WIT/A\&PCT system is similar to LLNL's IMPACT system with some minor variations. The WIT/A\&PCT staging rotates and elevates the drum. For ACT the source and detector are pair-wise translated instead of the drum. For PCT only the detector is translated. There is no difference in the data that is stored when translating the source/detector pair instead of the drum.

Due to spatial limitations within the WIT trailer it was not possible to use a detector collimator with an aspect ratio of $5: 1(5.2-\mathrm{cm} \times 5.2-\mathrm{cm}$ square aperture and $26-\mathrm{cm}$ length). A detector collimator with an aspect ratio of 2.5:1 was designed with the provision of using septa inserts to increase the aspect ratio to obtain the required collimator aspect ratio of 5:1 or greater. Septa are highly attenuating, dividing-plates that run the length of the collimator. These plates help collimate the gamma-ray beam, and provide an effective aspect ratio that is better than that provided by the aperture size alone. All other parameters of the WIT scanner are the same as those used for the IMPACT scanner.

\section{II.C.2.3 Data Reduction}

All WIT / APCT performance data shown here is reduced using the UCSFMLEM image reconstruction and assay code. The performance data acquired and shown for WIT/APCT was obtained over several years. We did not use the newly developed APCT-MLEM or APCT-CCG codes since they were not available until just recently, and they have not yet been implemented on the WIT trailer data-reduction computer. Since we did not have the time to run the new codes on the WIT/APCT data, it is important to note once again that we show only the UCSF-MLEM performance results.

\section{II.C.2.4 Calibration of WIT/A\&PCT}

It is important to emphasize that the objective for the WIT/A\&PCT scanner like the IMPACT scanner goal was to design a gamma-ray-based, waste-drum nondestructive assay technology that does not require special 
calibration as a function of the type or distribution of waste matrix, or the type or distribution of gamma-ray radioactive sources within the drum. This technology does not require prior knowledge of, or calibration for, the waste stream that is being assayed. The A\&PCT technology only requires an understanding of the physics of the measurement process and the absolute efficiency of the HPGe detector to provide a quantitative nondestructive assay measurement of all sorts of waste drums.

To obtain an absolute assay measurement, the WIT/A\&PCT system is calibrated once on an absolute detector-efficiency scale by simple measurements of a calibrated radioactive point source. We do not need additional calibrations for different $\mathrm{Pu}$ gram-amounts or for different types of attenuating waste matrices since they are measured in the A\&PCT gamma-ray method of nondestructive assay.

For the WIT/A\&PCT system a similar point source calibration method to that used for IMPACT is employed; however it is slightly complicated by the use of septa in the shorter WIT/A\&PCT detector collimator. The primary difference in the calibration method is that the IMPACT response function is calculated for a square aperture detector collimator, while for WIT/A\&PCT we determine the response function through a set of measurements. The response functions are obtained from a series of measurements with the source on the center axis of rotation and displaced from this axis by various amounts. Typically, we measure three response functions-near, center and far approximately 40,60 and $80 \mathrm{~cm}$ from the detector collimator front facewith a ${ }^{152} \mathrm{Eu}$ source. The appropriate measured geometric response function (typically the $344-\mathrm{keV}$ peak from ${ }^{152} \mathrm{Eu}$ is used) is used instead of the calculated response function in the image reconstruction and assay code. We also measure the detector efficiency from a calibrated, point source of multiple energy gamma-rays.

\section{II.C.2.5 Validation of WIT/A\&PCT}

The following is the verification procedure used for the single-HPGe detector WIT/A\&PCT system. This procedure will also be used in the verification procedure for the six-HPGe detector system. Two $\sim 10-\mu \mathrm{Ci}$ NIST traceable ${ }^{152} \mathrm{Eu}$ and ${ }^{133} \mathrm{Ba}$ sources are placed in the mid-plane of the combustible (top) layer of the BIR mock-waste drum. The drum is placed on the staging system of the WIT trailer with the seam of the drum facing the 2 $\mathrm{MeV}$ x-ray source as shown in Figure II.C.2-2.

$\mathrm{A}^{133} \mathrm{Ba}$ and ${ }^{152} \mathrm{Eu}$ source holder was designed and constructed so that the two sources extend half way into the combustible layer (top third) of the drum. The source holder provides positioning that is repeatable to within approximately $\pm 3.2 \mathrm{~mm}(1 / 8$-in.). A technique was developed to insure that the verification drum is placed in the same position (repeatable to better than $\pm 3.2 \mathrm{~mm}$ ) for each validation check. After positioning the drum onto the rotating table, active and passive tomography scans are performed for one 
slice centered on the two emission sources. All other test parameters are the same as those used in any actual drum assay.

Image reconstruction and assay is routinely performed on several of the isotopic peaks for each NIST source. The final attenuation corrected activity is compared to the actual known value of the two sources (correcting for any half-life decay) and to any previous measurements. A visual inspection of the reconstructed emission image also identifies any problems with the assay equipment and software. The reconstructed images generally should be the

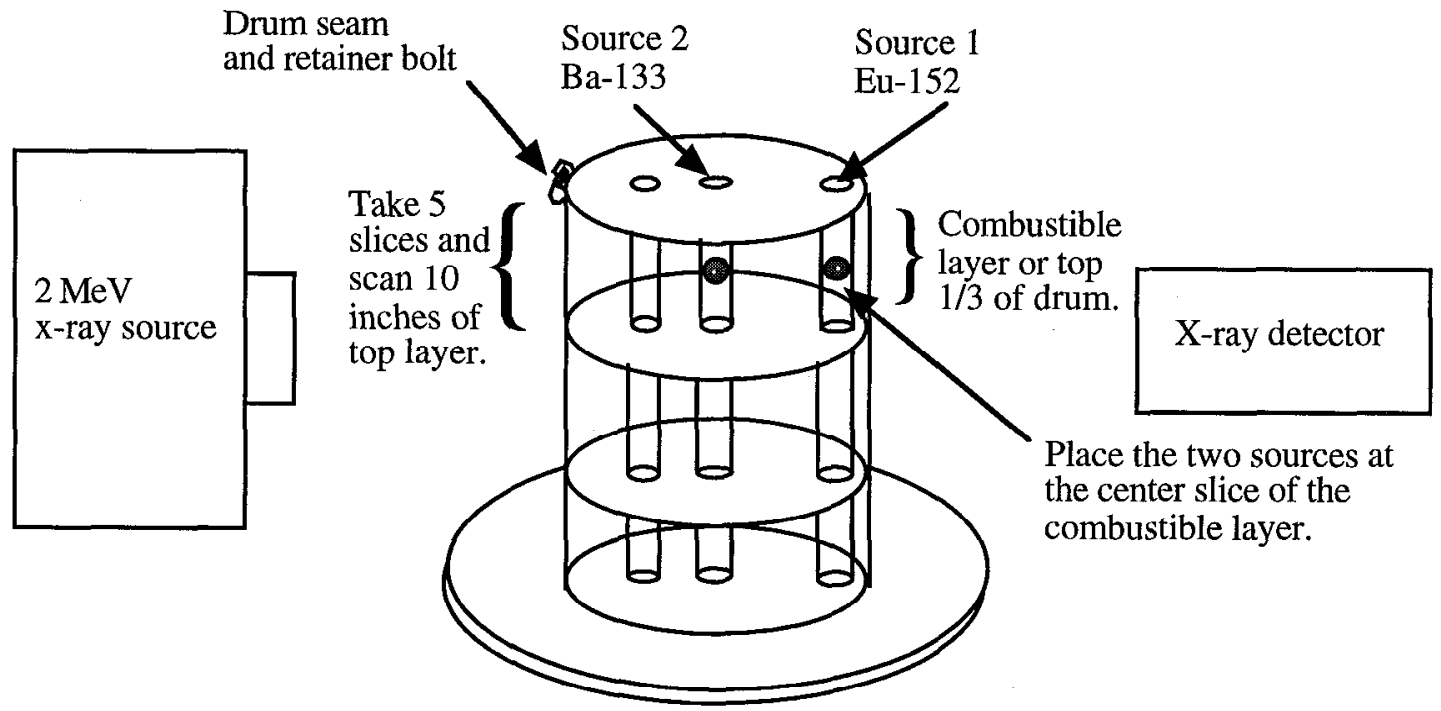

Figure II.C.2-2: Schematic of the drum and WIT/A\&PCT validation measurement procedure for BIR's mobile WIT trailer.
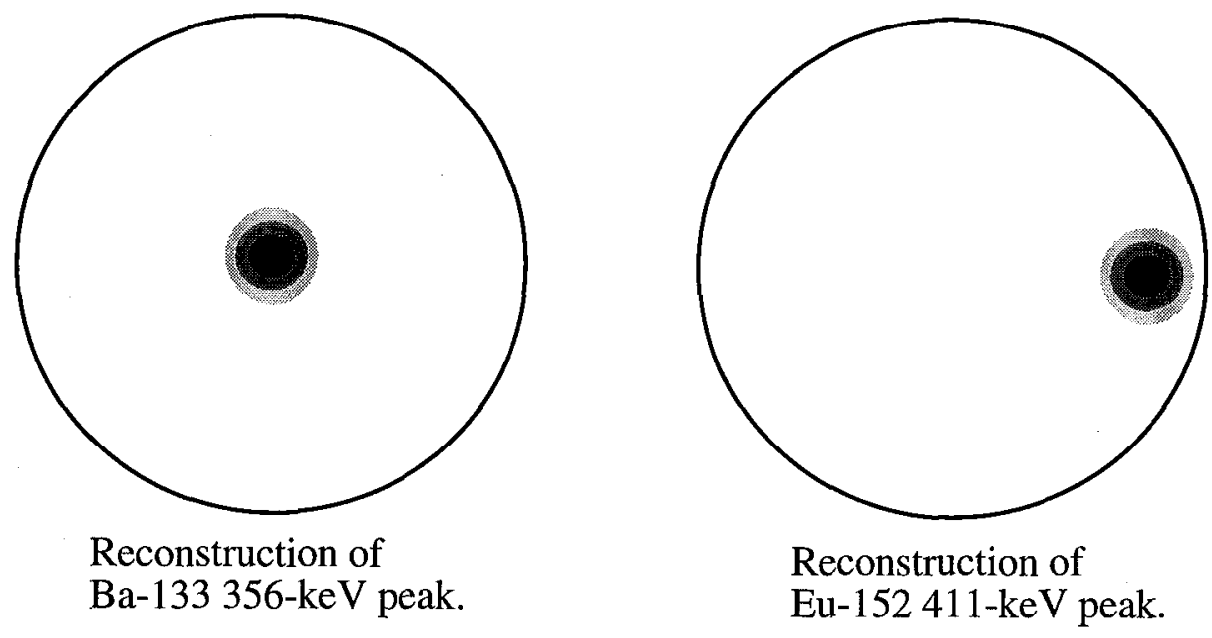

Figure II.C.2-3: Schematic of the expected CT slice results for the BIR WIT/A\&PCT validation procedure as shown in Figure II.C.2-2. 
same for each verification scan if the sources and drum are placed in the same position prior to the examination and all positions were reproduced as designed. An example of idealized reconstructed emission images are shown in Figure II.C.2-3. A combination of comparing the measured reconstructed assay values to known and previously measured values and verifying visually the placement of the two sources should reveal any significant problem that might exist in the A\&PCT system.

\section{A\&PCT Performance}

We have carried out numerous tests of the A\&PCT technology on surrogate and real-TRU waste drums. Both the IMPACT and the WIT /A\&PCT systems were calibrated, validated and used to measure real wastes at the LLNL. WIT/A\&PCT was demonstrated, tested and evaluated at three sites: (1) The Rocky Flats Environmental Technology Site (RFETS); (2) Two separate occasions at the INEEL; and (3) Most recently at the Nevada Test Site.

At LLNL, many of the drums contained multiple 4 and 20-liter (1 and 5gallon) containers of solidified chemical radioactive wastes. The RFETS wastes were all low-density combustible matrices. At INEEL the wastes included both lead-lined and normal drums containing waste matrices of graphite, glass, metals, wet and dry combustibles and sludge. The plutonium loading of these drums ranged from $\sim 1$ to 100 grams of ${ }^{239} \mathrm{Pu}$.

For this report, it is most uscful to specify the $\Lambda \& P C T$ performance as a function of known waste configurations and emission sources assayed using both the IMPACT and WIT/A\&PCT scanners. It is important to note that these two scanners do not use exactly the same configuration over all testing campaigns. Since the work presented here has mainly been an R\&D effort, the scanner configurations including hardware and software has evolved over time and it would be very difficult to describe all of the exact configurations used throughout all of the test data sets shown. However, they do not differ in any significant ways that would invalidate intercomparison. In an attempt to clarify the situation, the tabulated performance results are based only on the UCSF-MLEM image reconstruction and assay algorithm and is indicated in each table. Requirements and performance criteria used to evaluate IMPACT and BIR WIT/A\&PCT are presented in the next section.

\section{III.A Requirements and Performance Criteria}

An NDA waste assay system's utility is defined in terms of its ability to comply with the requirements and quality assurance objectives for nondestructive assay as delineated in the National TRU Program (NTP) Quality Assurance Program Plan (QAPP).[QAP96] The QAPP identifies the quality of data necessary to meet the specific data quality objectives associated with the Department of Energy's Waste Isolation Pilot Plant (WIPP) 
transuranic (TRU) waste characterization program. The primary parameter that must be determined is total TRU-alpha activity. The quality assurance objectives (QAOs) for precision (percent relative standard deviation-\%RSD), accuracy (percent recovery-\%R), minimum detectable concentration (MDC), completeness, and total bias are stated in the QAPP. These parameters must be demonstrated over the spectrum of waste-form configurations the assay system is intended to characterize.

The QAPP also requires that facilities intending to use NDA methods to generate data for the National TRU program participate in a Performance Demonstration Program (PDP). The PDP program is designed as an independent quality assurance test to provide data that supports the overall QAPP compliance assessment process. The PDP program parameters, criteria and scoring formalism where applicable are applied in the following sections to document the IMPACT and WIT/A\&PCT performance. The PDP, Rapid Commercialization Initiative (RCI) and Capability Evaluation Project (CEP) performance tests, requirements and criteria are described below.

Utility is further evaluated relative to the spectrum of waste form configurations that the different assay system techniques can accommodate. The performance of IMPACT and WIT/A\&PCT are derived from data acquired through a blind testing process. The performance evaluation tests being derivatives of QAPP QAOs support a direct interpretation of IMPACT and WIT/A\&PCT performance results. Thus, the IMPACT and WIT/A\&PCT performance measures can be readily interpreted relative to the National TRU Program QAPP requirements. The criteria of these various programs are listed next.

\section{III.A.1 Quality Assurance Program Plan-Table 9.1}

The performance assessment parameters and evaluation criteria as found in the NTP program QAPP, Section 9.0, Interim Change are presented in Table III.A-1. All measurement series that were acquired per the prescriptions (e.g., 15 replicate non-interfering precision and accuracy parameters) are evaluated via the applicable criteria listed within Table III.A-1.

\section{III.A.2 Performance Demonstration Program}

The NDA PDP is an independent quality assurance test of assay system performance.[PDP97, MAZ97] Data generated during PDP test cycles is also considered in the overall QAPP compliance assessment process. The PDP consists of periodic tests conducted of both increasing matrix and source complexities to evaluate the capability of various technologies to properly characterize TRU waste throughout the DOE complex. Each test is termed a PDP cycle. These evaluation cycles are blind tests that provide an objective measure of the reliability and performance of the various NDA systems. The PDP test samples are comprised of 208- $L$ drums configured with NIST traceable Working Reference Materials (WRMs). The PDP test on any given 
Table III.A-1 QAPP quality assurance objectives for nondestructive assay.

\begin{tabular}{|c|c|c|c|c|}
\hline $\begin{array}{c}\text { Waste Activity } \\
\text { alpha-Ci range }^{\mathrm{a}}\end{array}$ & $\begin{array}{c}\text { Nominal } \\
\text { Compliance Point, } \\
\text { alphu-Ci (g WG Pu) }\end{array}$ & $\begin{array}{c}\text { Precision }^{\mathrm{c}} \\
(\% \mathrm{RSD})\end{array}$ & $\begin{array}{c}\text { Accuracy }^{\mathrm{d}} \\
(\% \mathrm{R})\end{array}$ & $\begin{array}{c}\text { Total Bias } \\
(\%)\end{array}$ \\
\hline$>0.002-0.02$ & $0.008(0.1)$ & $\leq 20$ & $75-125$ & $\begin{array}{c}\text { low } 25 \\
\text { high } 400\end{array}$ \\
\hline$>0.02-0.2$ & $0.08(1.0)$ & $\leq 15$ & $50-150$ & $\begin{array}{c}\text { low } 35 \\
\text { high } 300\end{array}$ \\
\hline$>0.2-2.0$ & $0.8(10)$ & $\leq 10$ & $75-125$ & $\begin{array}{c}\text { low } 67 \\
\text { high } 150\end{array}$ \\
\hline$>2.0$ & $12.5(160)$ & $\leq 5$ & $75-125$ & $\begin{array}{c}\text { low } 67 \\
\text { high } 150\end{array}$ \\
\hline \multicolumn{4}{|c}{ Minimum Detectable Concentration (nCi/g) ---60 (100) } \\
\hline
\end{tabular}

a. Applicable range of TRU activity in a 208-liter (55-gallon) drum to which the QAOs apply. Units are Curies of alpha-emitting TRU isotopes with half-lives greater than 20 years.

b. The nominal activity (weight of $\mathrm{Pu}$ ) in the 208-liter ( 55 gallon) drum used to demonstrate that QAOs can be achieved for the corresponding range in column 1 . Values in parentheses are the approximate equivalent weights of weapons grade plutonium (WG Pu), fifteen years after purification. For purposes of demonstrating QAOs, "nominal" means + 10 percent.

c. Plus or minus one standard deviation based on fifteen replicate measurements of a noninterfering matrix. d. Ratio of measured to known values based on the average of fifteen replicate measurement of a noninterfering matrix.

e. 95 percent confidence bounds for system bias established by studies to determine contributions to total uncertainty from all significant sources. Units are confidence bounds divided by true value, expressed as a percent. Requirement for the QAO for total measurement uncertainty is to determine and document, but no DOE system-wide values have been established.

test sample requires six replicate measurements and removal of the drum between each replicate measurement.

Presently there are five drum matrices: air (no matrix), ethafoam ${ }^{10}$, combustibles, glass, and inorganic sludge. Aluminum source insert fixtures are provided for each the insert tube radii. NIST traceable WRMs are positioned at desired vertical locations within the insert fixtures. Several types of WRMs are used in the program, e.g., WG Pu, large particle WG Pu and enriched U. The initial WRMs for the first four cycles were weapons-grade plutonium dioxide $\left(\mathrm{PuO}_{2}\right)$ uniformly mixed in diatomaceous earth and then encapsulated in a dual stainless steel cylinder configuration $(o . d .: 5 \mathrm{~cm}, l: 23 \mathrm{~cm})$. Currently five cycles have been completed. The A\&PCT technology participated in and passed cycle 2 informally, and formally cycles 3 and 4 .

${ }^{10}$ This drum martix is not used in the PDP any more. 
Table III.A-2 Summary of Performance Demonstration Program (PDP) criteria.

\begin{tabular}{|c|c|c|c|c|}
\hline $\begin{array}{c}\text { Range of } \\
\text { Waste } \\
\text { Activity } \\
(\alpha \text {-Curies) }\end{array}$ & $\begin{array}{c}\text { Nominal } \\
\text { Compliance } \\
\text { Point } \\
\alpha \text {-Curies } \\
(\mathbf{g} \text { WG Pu) }\end{array}$ & $\begin{array}{c}\text { Relative } \\
\text { Precision } \\
\left(\mathbf{R}_{\mathrm{p}}\right)\end{array}$ & $\begin{array}{c}\text { Instrument } \\
\text { Bias } \\
(\% \mathbf{R})\end{array}$ & $\begin{array}{c}\text { Total } \\
\text { Bias } \\
\mathbf{( \% )}\end{array}$ \\
\hline$>0-0.02$ & $\begin{array}{c}0.008 \\
(0.1)\end{array}$ & 40 & $75-125$ & $\begin{array}{c}\text { Low } 40 \% \\
\text { High } 175 \%\end{array}$ \\
\hline$>0.02-0.2$ & $\begin{array}{c}0.08 \\
(1.0)\end{array}$ & 30 & $50-150$ & $\begin{array}{c}\text { Low } 30 \% \\
\text { High } 200 \%\end{array}$ \\
\hline$>0.2-2.0$ & 0.8 & 20 & $50-150$ & $\begin{array}{c}\text { Low } 30 \% \\
\text { High } 200 \%\end{array}$ \\
\hline$>2.0$ & $(10)$ & 10 & $75-125$ & $\begin{array}{c}\text { Low } 50 \% \\
\text { High } 150 \%\end{array}$ \\
\hline
\end{tabular}

All measurement series that were acquired per the PDP (e.g., 6 replicate precision and bias parameters) are evaluated via the applicable criteria listed within Table III.A-2. IMPACT and WIT/A\&PCT participated in and passed PDP cycle 3 and 4, respectively.

\section{III.A.3 Capability Evaluation Project}

As part of the MWFA characterization development strategy, a method to objectively evaluate the utility of waste-assay system technologies was implemented in conjunction with the Characterization Monitoring and Sensor Technology (CMST) crosscut area program. This evaluation was designed to support nondestructive waste assay system technology capability and deficiency determinations, and to facilitate resource allocation to areas requiring development. The evaluation was also intended to generate information and data to end-user EM-30 Waste Management programs to support appropriate selection and application of a given nondestructive assay technology to the various waste streams.

The Capability Evaluation Project (CEP) was specified in a manner such that evidence is derived to substantiate nondestructive waste assay capability and utility statements as a function of waste type and/or characteristic. The waste types for which the evaluation is conducted are those contaminated with transuranic elements. The evaluation program was conducted at the INEEL Radioactive Waste Management Complex (RWMC) using actual waste forms currently in storage and carefully specified and constructed surrogates. To the extent RWMC waste form attributes approximate other site waste inventories, statements can also be made regarding system utility per the site of interest. The capability evaluation plan addressed the acquisition, compilation and reporting of performance data, thereby allowing a given 
agency a basis for an objective evaluation of participating waste NDA systems. The evaluation was structured such that a statement regarding select INEEL RWMC waste forms can be composed relative to compliance potential for applicable National TRU Program requirements and criteria.

The test is designed to provide objective and unbiased data regarding the performance and associated capability of each participating mobile assay system to the MWFA, to the CMST, and to procurers of waste assay system services and technology holders. The test series consists of a combination of surrogate waste-form and actual waste-form test samples. The surrogate-type test samples allow an evaluation of assay system performance where the matrix and radioactive source constituents and configurations are accurately known. The actual waste-type test samples are the unique aspect of the CEP in that performance is assessed with respect to the actual waste forms and their associated configurations.

Criteria used to evaluate assay system capability are founded in the NTP program QAPP, Section 9.0, Interim Change version, the Performance Demonstration Program Plan for Nondestructive Assay for the TRU Waste Characterization Program. Applicable criteria can be readily applied to the surrogate-type test samples because the alpha activity placed within the surrogate matrix is well known. This allows the accepted scoring formalism of the NDA PDP program to be utilized in the CEP performance evaluation process with minor modifications; thus simplifying interpretation and the derivation of compliance/performance statements.

Table III.A-3 Measured relative precision requirement adjusted for eight replicates used in the CEP.

\begin{tabular}{|c|c|c|c|}
\hline $\begin{array}{c}\text { Activity } \\
\text { Range in } \\
\text { Curies }\end{array}$ & $\begin{array}{c}\text { Maximum Allowable } \\
\text { Precision } \\
\left(95 \% \mathrm{CB}^{\mathrm{a}} \text { of QAPP QAO) }\right.\end{array}$ & $\begin{array}{c}\text { Maximum Measured } \\
\text { Precision (\%RSD) } \\
\text { @ 8 replicates } \\
\text { (noninterfering) }\end{array}$ & $\begin{array}{c}\text { Maximum Measured } \\
\text { Precision (\%RSD) } \\
@ \text { 8 replicates } \\
\text { (interfering) }\end{array}$ \\
\hline $\begin{array}{c}>0 \text { to } \\
0.02\end{array}$ & 29.2 & $\leq 16.0$ & $\leq 18.0$ \\
\hline $\begin{array}{c}>0.02 \text { to } \\
0.2\end{array}$ & 21.9 & $\leq 12.0$ & $\leq 14.0$ \\
\hline $\begin{array}{c}>0.2 \text { to } \\
2.0\end{array}$ & 14.6 & $\leq 8.0$ & $\leq 14.0$ \\
\hline$>2.0$ & 7.3 & $\leq 4.1$ & $\leq 7.0$ \\
\hline
\end{tabular}

Note: ${ }^{a} \mathrm{CB}$ is Confidence Bound 
The precision and bias QAOs are based on the NDA PDP Program QAOs. Modification of the NDA PDP QAOs has been performed to account for the number of replicates (eight) utilized per sample in the CEP project. The NDA PDP noninterfering and interfering matrix QAOs for precision are tabulated in Table III.A-3. The noninterfering and interfering matrix QAOs for bias used in the CEP are derived from the PDP (Table III.A-2) and are tabulated in Table III.A-4.

Table III.A-4 CEP bias QAOs taken from PDP.

\begin{tabular}{|c|c|c|}
\hline $\begin{array}{c}\text { Activity } \\
\text { Range in } \\
\text { Curies }\end{array}$ & $\begin{array}{c}\text { Bias } \mathrm{QAO} \\
\text { Values for } \% \mathrm{R}_{\mathrm{L}} \text { and } \% \mathrm{R}_{\mathrm{U}} \\
\text { (noninterfering) }\end{array}$ & $\begin{array}{c}\text { Bias } \mathrm{QAO} \\
\text { values for } \% \mathrm{R}_{\mathrm{L}} \text { and } \% \mathrm{R}_{\mathrm{U}} \\
\text { (interfering) }\end{array}$ \\
\hline$>0$ to 0.02 & Low: $75 \%$ & Low: $40 \%$ \\
& High: $125 \%$ & High: $175 \%$ \\
\hline$>0.02$ to 0.2 & Low: $50 \%$ & Low: $30 \%$ \\
& High: $150 \%$ & High: $200 \%$ \\
\hline$>0.2$ to 2.0 & Low: $75 \%$ & Low: $30 \%$ \\
& High: $125 \%$ & High: $200 \%$ \\
\hline$>2.0$ & Low: $75 \%$ & Low: $50 \%$ \\
& High: $125 \%$ & High: $150 \%$ \\
\hline
\end{tabular}

\section{III.A.4 Rapid Commercialization Initiative Test}

Bio-Imaging Research Inc. was engaged in a Program Research and Development Agreement (PRDA) and a Rapid Commercialization Initiative with the Department of Energy, EM-50. The agreement requires BIR to develop information sufficient to establish compliance with applicable National TRU Program waste characterization requirements and associated quality assurance performance criteria. This effort requires an objective demonstration of the BIR waste characterization system. As with the CEP project, the goal of the RCI test project is to provide a mechanism from which evidence can be derived to substantiate nondestructive assay capability and utility statements for the BIR system. The performance evaluation parameters and criteria used in the RCI project are as indicated for the CEP. Similar to the CEP test project, the RCI test utilized test samples with configurations representative of a large population of waste types in inventory at the INEEL RWMC. 


\section{III.B IMPACT Test Descriptions and Performance}

All data presented in this section were obtained using the UCSF-MLEM image reconstruction and assay codc. During PDP cycle 2 we were not ready to officially participate, but we were able to gain access to one of the two drums. This drum has an ethafoam matrix and was loaded with 4 PDP standards (three $0.3 \mathrm{~g}$ and one $0.03 \mathrm{~g}$ of WG Pu).[PDP97a] The attenuation caused by the drum and ethafoam matrix was measured by ACT, which required 17 slices with a ray sum integration time of $10 \mathrm{~s}$.

We ran several complete-sampled PCT scans (14 ray sums, 21 projections, and 17 slices) with different ray sum integration times as shown in Figure III.B-1. This data showed that as the integration time decreased the assay result increased. The increasing amounts of additional $\mathrm{Pu}$ mass is attributed to a poor counting statistics resulting in an incorrect use of the background in the passive sinogram data since the MLEM algorithm does not accept negative values (see Section II.B.3). Thus, for signals that approach a background level of zero we end up with a positive background overall since the negative values are set to zero and this results in a positive assay bias when using the MLEM optimization algorithm. Methods applied to account for this bias are not always successful; thus, we developed the CCG method as described in Section II.B.3. Until this algorithm research is completed we need to use integration times that are sufficiently long to provide a background

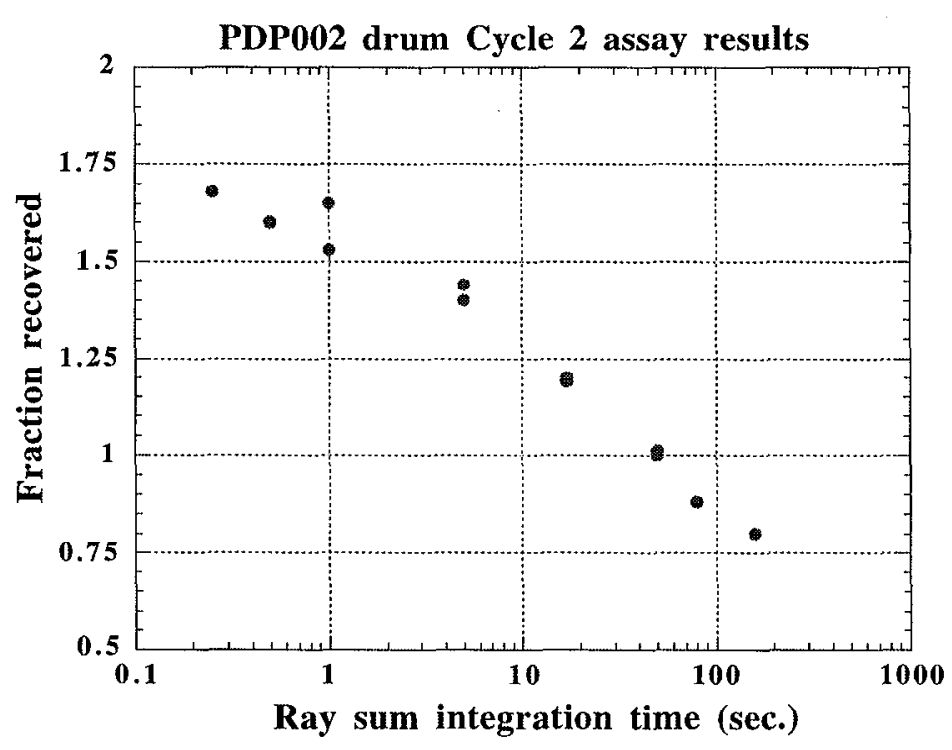

Figure III.B-1 Summary of the IMPACT UCSF-MLEM assay results as a function of ray-sum integration time for PDP cycle 2 ethafoam-matrix (PDP002) drum with $0.93 \mathrm{~g}$ of WG Pu. Some integration times were repeated two or three times. Fraction recovered is our measured result relative to true. 
above zero and a signal that is above the background. Of course, the integration time required is a direct function of the activity of the drum and its attenuating matrix. For example, we show below (PDP cycle 3 ) that for high activity drums we've not yet seen this positive bias at short integration times.

A 15 measurement replicate study was completed for one of the four QAPP activity ranges. Three PDP standards (one $3 \mathrm{~g}$ and two $0.3 \mathrm{~g}$ of WG Pu) were loaded into the combustible (PDP003) matrix drum. For all scans 9 slices were obtained. The ACT ray sum integration time was $15 \mathrm{~s}$. Results for a complete-sampled (14 ray sums, 21 projections, and 9 slices with a ray sum integration time of $25 \mathrm{~s}$ ) PCT data set and an under-sampled PCT data set are shown in Figure III.B-2. The complete-data set results in a total bias or $\% \mathrm{R}$ of 80.4 with a \%RSD of 2.8 , which meets QAPP requirements.

The under-sampled data were obtained by computationally removing every other ray and projection from the complete-sample data set. This results in a $4 \mathrm{X}$ reduction of the complete-data set or 7 ray sums, 9 projections and 9 slices. The under-sampled data \%RSD is 3.9 with a $\% R$ of 79.0 . Since the difference in these two data sets is $<2 \%$ and both meet QAPP requirements, we conclude that we can use the under sampling protocol to assay a drum, and do it in one quarter the passive data acquisition time. The under-sampled results were verified experimentally in subsequent tests (not discussed here).

LLNL formally participated in and passed PDP cycle 3 . This test consisted of the combustible-matrix drum with 7 PDP standards $(50,15$, two $3,0.3$ and two $0.03 \mathrm{~g}$ ) resulting in a total of $71.36 \mathrm{~g} \mathrm{WG} \mathrm{Pu}$; and the glass-matrix drum with 4 PDP standards having a total of $98.3 \mathrm{~g}$ WG Pu.[PDP97b] All ACT scans consisted of 17 slices with a ray-sum integration time of $6 \mathrm{~s}$.

The PCT data consisted of 7 ray sums, 9 projections and 17 slices with a $20 \mathrm{~s}$ ray sum integration time. Our assay results are summarized in Figure III.B-3. For the drum with the combustible matrix (71.36 $\mathrm{g} \mathrm{WG} \mathrm{Pu),} \mathrm{the} \mathrm{measured}$ mean is $47.1 \mathrm{~g}$. The total bias (\%R) is 66.0 with a \%RSD of 0.58 . For the drum with the glass matrix (98.3 $\mathrm{g} \mathrm{WG} \mathrm{Pu}$ ), the measured mean is $69.9 \mathrm{~g}$, a total bias of 71.1 with a \%RSD of 0.84 . The average of the total bias and \%RSD is 68.5 and 0.7 , respectively. Therefore, the bias of IMPACT seems to be about $30 \%$ low for all mass amounts. A representative 3-D surface rendered PCT image of the ${ }^{239} \mathrm{Pu}$ source distributions in the PDP cycle 3 glass-matrix drum (PDP004) is shown in Figure III.B-4. 
Replicate scans of PDP 003 (combustibles) with $3.6 \mathrm{~g}$ WG Plutonium

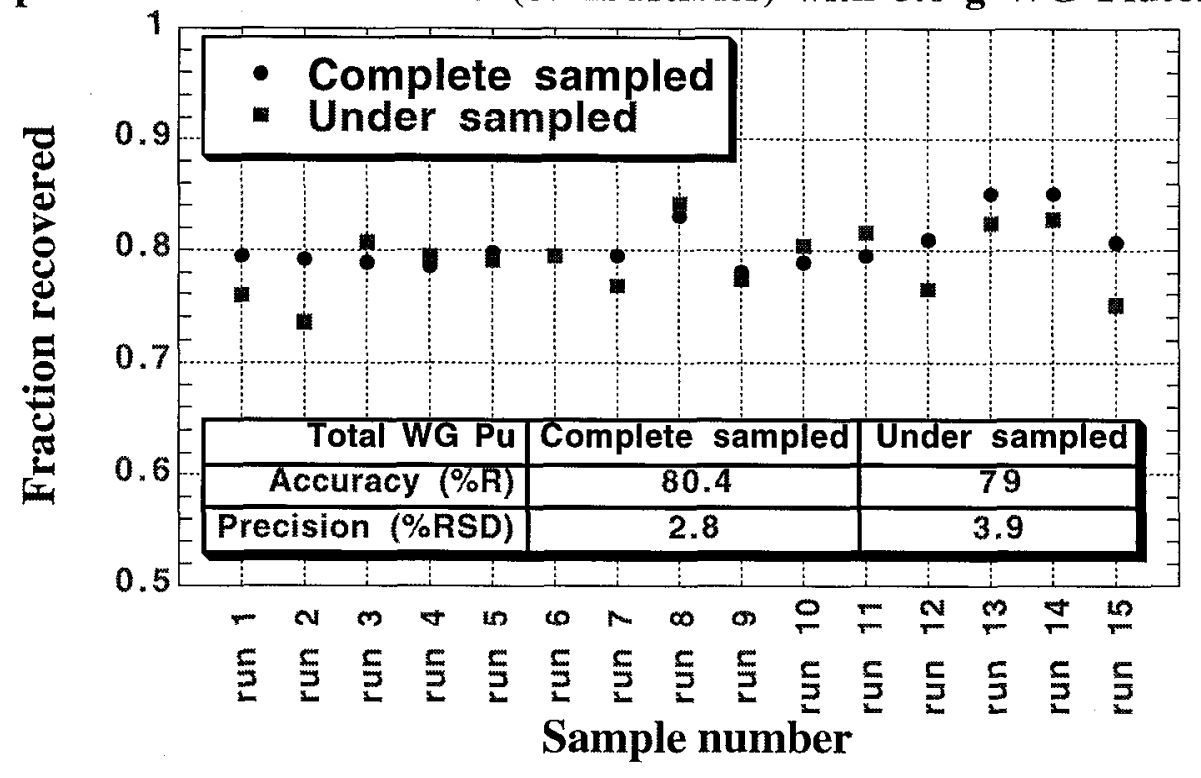

Figure III.B-2 Summary of IMPACT UCSF-MLEM 15 replicate measurements for the combustible-matrix (PDP003), 3.6 g WG Pu drum. Complete- and under-sampled (by a factor of 4 ) data are shown for comparison.

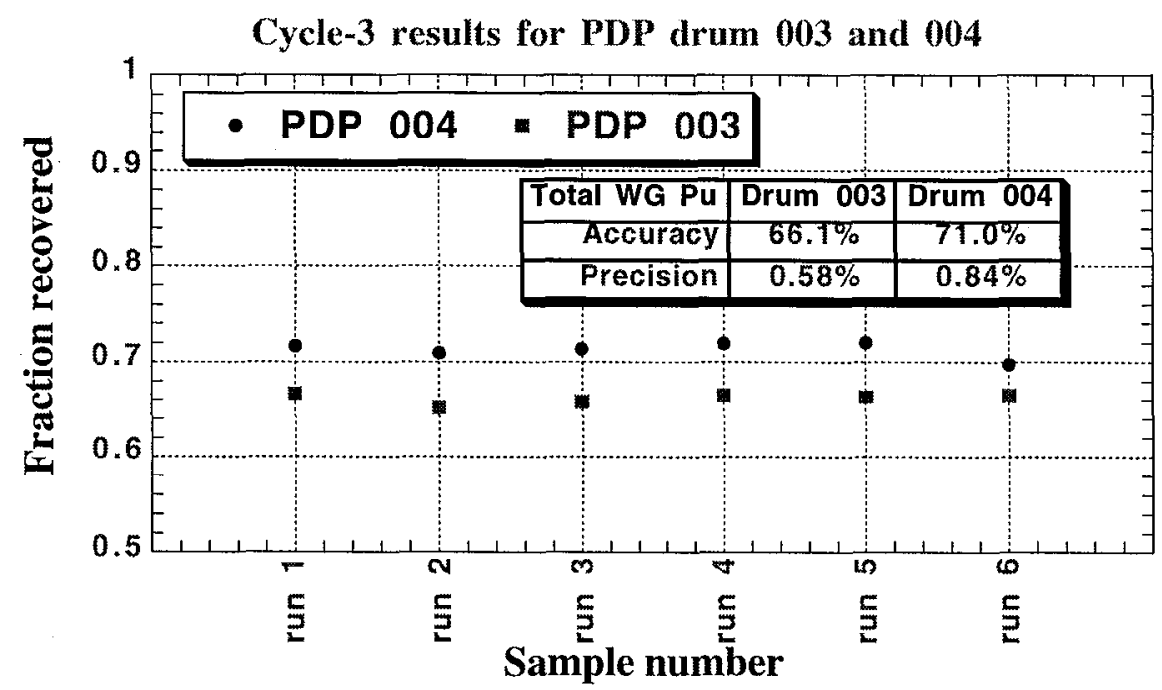

Figure III.B-3 Summary of the IMPACT UCSF-MLEM assay results for PDP cycle 3 combustible (PDP003) and glass (PDP004) matrices with 71.36 and $98.3 \mathrm{~g}$ of WG Pu, respectively. 


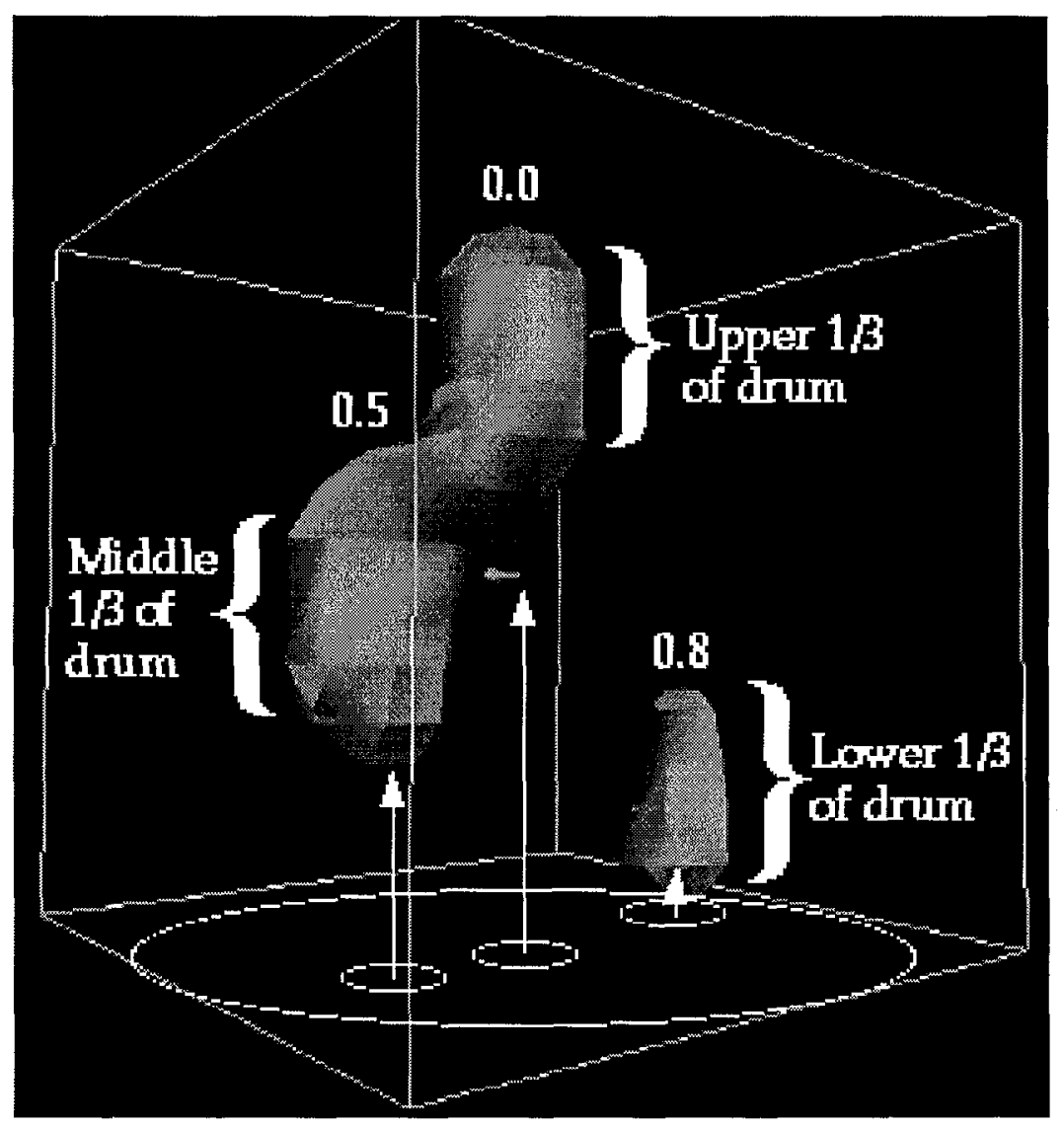

Figure III.B-4 Representative 3-D surface rendered PCT image of the ${ }^{239} \mathrm{Pu}$ source distribution in the PDP cycle 3 glass-matrix drum. The standards within this drum (PDP004) consisted of WG Pu: $65 \mathrm{~g}$ at $0 \mathrm{R}$ ( $\mathrm{R}$ is the drum radius), $30 \mathrm{~g}$ at $0.5 \mathrm{R}, 3 \mathrm{~g}$ at $0.8 \mathrm{R}$, and $0.3 \mathrm{~g}$ at $0 \mathrm{R}$ at a height from the bottom of the drum of $40.6,17.8,0$, and $12.7 \mathrm{~cm}$, respectively. Note that 3 of the 4 standards were recovered; however, the smallest standard was not recovered.

Two additional measurements were made on the glass matrix to better understand the system bias at short integration times. PCT measurements at 10 and $5 \mathrm{~s}$ ray-sum integration times gave 69.4 and $69.1 \mathrm{~g}$, respectively. These results agree with the $20 \mathrm{~s}$ PCT assay to within 1\%. Thus, for signals above the system noise our assay results are independent of ray-sum integration times.

\section{III.C WIT/A\&PCT Test Descriptions and Performance}

All data presented in this section were obtained using the UCSF-MLEM image reconstruction and assay code. The WIT scanner formally participated in and passed the CEP, RCI, and PDP Cycle-4 performance tests. For all tests it was important to assay as many drums as possible in a limited amount of 
time. Thus it was decided to acquire and analyze A\&PCT data in $~ 22$ hours. This allowed BIR to acquire both NDE and NDA data for each drum within a 24 hour period. This was imposed by field and test conditions. Occasionally, we were able to run a drum over a weekend. We used this longer assay time to acquire data on high-attenuating and/or low-activity waste drums. This data was acquired only by BIR personnel (a technician). BIR allowed LLNL to look at all A\&PCT data acquired and analyzed on the WIT trailer. For the RCI test we manually analyzed the PCT spectroscopy data to provide isotopic analysis for the final assay report. In the CEP and PDP data analysis we assumed that the waste forms assayed contained only weapons grade $\mathrm{Pu}$.

The CEP performance measures consisted of drums with metals, molten salt extraction (MSE) salts, Raschig rings, and sludge loaded with 3.97, 46.0, 0.961 , and $1.53^{11} \mathrm{~g}$ of ${ }^{239} \mathrm{Pu}$, respectively. The CEP test protocol required the measurement of eight replicates on each test drum. The replicate data allows for the determination of an average \%R and \%RSD for each test drum. The performance results for the WIT/A\&PCT technology as determined by the CEP evaluation are shown in Table III.C.-1. The CEP test drum total alphaCurie average \%R and \%RSD is $125 \%$ and $2.9 \%,{ }^{12}$ respectively. Therefore, the CEP test bias of WIT / A\&PCT is about $25 \%$ high.

The RCI surrogate drums contained glass, combustible, and metal matrices with 2.22, 0.933, and $0.747 \mathrm{~g}$ of ${ }^{239} \mathrm{Pu}$ and $6.96,1.01$ and $0.804 \mathrm{mg}$ of ${ }^{241} \mathrm{Am}$, respectively. The RCI actual TRU waste drums contained graphite, combustible, Raschig rings, and filters with $24.4, \sim 0.02,10.1$, and $76.3 \mathrm{~g}$ of ${ }^{239} \mathrm{Pu}$, and $36,0.03,50$, and $280 \mathrm{mg}$ of ${ }^{241} \mathrm{Am}$, respectively. The actual waste drum mass values are based on best estimates as acquired via the SWEPP NDA system and therefore have an uncertainty with them. Due to time limitations associated with the RCI project, only a single measure was acquired for each test drum, i.e., no replicates. Precision for WIT necessary for scoring the RCI data was acquired from other measurement series, e.g., IMPACT and WIT / A\&PCT QAO testing, PDP and CEP participation. The \%RSD determined from these other tests were correlated to the appropriate RCI test drum with similar mass amounts. The WIT/A\&PCT performance results are shown in Table III.C-2. The inorganic sludge data is not fully evaluated at this time. The average of the RCI total alpha-Curie \%R and \%RSD is 119 and 3.8, respectively. Therefore, the composite bias of WIT/A\&PCT for the RCI test is $\sim 20 \%$ high.

\footnotetext{
${ }^{11}$ This value is the average of two different assay measurements-the radiochemistry and SWEPP active neutron-based ND $\Lambda$ values of 1.35 and 1.71 grams of ${ }^{239} \mathrm{Pu}$, respectivcly.

${ }^{12}$ The RF11 drum has two assay values for which the WIT/A\&PCT results were compared (Table III.C-1). In our calculations we used the average \%R and \%RSD of the two RF11 values.
} 
Table III.C-1 BIR WIT/A\&PCT UCSF-MLEM performance on total alpha activity parameter for CEP test results.

\begin{tabular}{|c|c|c|c|c|c|c|c|c|c|c|}
\hline \multirow[t]{2}{*}{$\begin{array}{c}\text { Test } \\
\text { Drum ID }\end{array}$} & \multirow[t]{2}{*}{$\begin{array}{c}\text { Waste } \\
\text { IDC }\end{array}$} & \multirow[t]{2}{*}{$\begin{array}{c}\text { Precision } \\
\text { WIT \%RSD } \\
\left(\sigma / A_{t}\right)^{1}\end{array}$} & \multirow[t]{2}{*}{$\begin{array}{l}\text { Precision } \\
\text { on QAO } \\
(\% \text { RSD) }\end{array}$} & \multirow[t]{2}{*}{$\begin{array}{l}\text { Total } \alpha-\mathrm{Ci} \\
\text { avg. } \% \mathbf{R} \\
\left(A_{m} / A_{t}\right)^{1}\end{array}$} & \multicolumn{2}{|c|}{$\begin{array}{l}\text { Total } \alpha \text { avg. \% Recovery } \\
\text { Acceptance Criteria (95\% } \\
\text { Confidence Bounds) }\end{array}$} & \multirow[t]{2}{*}{$\begin{array}{l}{ }^{239} \mathrm{Pu} \\
\text { (avg. } \\
\% \mathrm{R})\end{array}$} & \multirow[t]{2}{*}{$\begin{array}{l}{ }^{241} \mathrm{~A} \mathrm{~m} \\
\text { (avg. } \\
\% \mathrm{R})\end{array}$} & \multirow[t]{2}{*}{$\begin{array}{l}{ }^{235} \mathrm{U} \\
\text { (avg. } \\
\% R \text { ) }\end{array}$} & \multirow[t]{2}{*}{$\begin{array}{l}{ }^{238} \mathrm{U} \\
(\text { avg. } \\
\% \mathrm{R})\end{array}$} \\
\hline & & & & & Lower \%R & Upper \%R & & & & \\
\hline \multicolumn{11}{|l|}{ Surrogates } \\
\hline SG6 & $\begin{array}{l}409 \\
\text { (MSE } \\
\text { Salts) }\end{array}$ & 1.1 & $<7.0$ & 70.7 & 50.9 & 149.1 & 103.5 & 15.4 & - & - \\
\hline SG9 & $\begin{array}{l}442 \\
\text { (Raschig } \\
\text { ring) }\end{array}$ & 4.2 & $<14.0$ & 154.9 & 33.5 & 196.5 & 146.0 & 284.4 & - & - \\
\hline \multicolumn{11}{|l|}{$\overline{\text { RFETS }}$} \\
\hline $\mathrm{RF} 11^{2}$ & $\begin{array}{c}003 \\
\text { (organic } \\
\text { sludge) }\end{array}$ & 5.0 & $<14.0$ & 161.4 & 35.0 & 195.0 & 144.9 & 141.9 & 0.0 & - \\
\hline$R F 11^{3}$ & $\begin{array}{c}003 \\
\text { (organic } \\
\text { sludge) }\end{array}$ & 5.9 & $<14.0$ & 191.0 & 35.9 & 194.1 & 183.8 & 242.9 & 0.0 & - \\
\hline RF20 & $\begin{array}{c}480 \\
\text { (metals) }\end{array}$ & 0.8 & $<14.0$ & 96.8 & 30.7 & 199.3 & 121.2 & 28.9 & 0.0 & 0.0 \\
\hline
\end{tabular}

Notes: $1 . A_{t}$ is the true activity and $A_{m}$ is the average measured activity

2. Active neutron-based measured mass evaluation

3. Radiuchemistry evaluation 
Table III.C-2 BIR WIT/A\&PCT UCSF-MLEM performance on total alpha activity parameter for RCI test results.

\begin{tabular}{|c|c|c|c|c|c|c|c|}
\hline \multirow[t]{2}{*}{$\begin{array}{l}\text { Test } \\
\text { Drum } \\
\text { ID }\end{array}$} & \multirow[t]{2}{*}{ Waste IDC } & \multirow{2}{*}{$\begin{array}{c}\text { Precision } \\
\text { WIT } \\
\% \text { RSD } \\
\left(\sigma / A_{t}\right)^{1}\end{array}$} & \multirow[t]{2}{*}{$\begin{array}{l}\text { Precision } \\
\text { QAO } \\
\text { (\%RSD) }\end{array}$} & \multirow[t]{2}{*}{$\begin{array}{c}\text { Total } \alpha-\mathrm{Ci} \\
\text { avg. } \% \mathbf{R} \\
\left(A_{m} / A_{t}\right)^{1}\end{array}$} & \multicolumn{2}{|c|}{$\begin{array}{c}\text { \% Recovery Acceptance } \\
\text { Criteria } \\
\text { (95\% Confidence Bounds) }\end{array}$} & \multirow[t]{2}{*}{$\begin{array}{l}{ }^{239} \mathrm{Pu} \\
(\% \mathbf{R})\end{array}$} \\
\hline & & & & & Lower \%R & Upper \%R & \\
\hline $1 \mathrm{RF}$ & 300 (graphite) & 7.1 & $<7.0$ & 127 & 57.4 & 142.6 & 122.0 \\
\hline $2 R F$ & $\begin{array}{c}336 \text { (moist } \\
\text { combustibles) }\end{array}$ & - & $<18.0$ & Below DL & - & - & $\begin{array}{l}\text { Below } \\
\text { DL }\end{array}$ \\
\hline $1 S G$ & 440 (glass) & 3.89 & $<14.0$ & 141.4 & 32.2 & 197.8 & 139.7 \\
\hline $3 R F$ & 442 (Raschig ring) & 2.95 & $<14.0$ & 122 & 33.1 & 196.9 & 133.0 \\
\hline $2 S G$ & $\begin{array}{c}330 \text { (dry } \\
\text { combustibles) }\end{array}$ & 4.15 & $<14.0$ & 162.5 & 32.5 & 197.5 & 149.9 \\
\hline $4 \mathrm{RF}$ & $\begin{array}{c}376 \\
\text { (filters/insulation) }\end{array}$ & 1.54 & $<7.0$ & 86 & 51.6 & 148.4 & 91.0 \\
\hline $3 S G$ & 480 (metals) & 4.15 & $<14.0$ & 179.6 & 33.5 & 196.5 & 174.0 \\
\hline $5 R F^{2}$ & $\begin{array}{l}001 \text { (inorganic } \\
\text { sludge) }\end{array}$ & 2.73 & $<7.0$ & 14.9 & 51.2 & 148.8 & 107.0 \\
\hline
\end{tabular}

Notes: $1 . A_{\text {i }}$ is the true activity and $A_{m}$ is the average measured activity.

2. $5 R F$ data not fully evaluated by LMITCO INEEL at this time. 
The PDP Cycle 4 performance measurements used the drums containing combustible and zero (or empty) waste matrices loaded with 6.66 (two $3 \mathrm{~g}$, two $0.3 \mathrm{~g}$ and two $0.03 \mathrm{~g}$ of $\mathrm{WG} \mathrm{Pu}$ ) and $98.3 \mathrm{~g}$ (one $50 \mathrm{~g}$, one $30 \mathrm{~g}$, one $15 \mathrm{~g}$, one $3 \mathrm{~g}$ and one $0.3 \mathrm{~g}$ of $\mathrm{WG} \mathrm{Pu}$ ) of WG Pu, respectively. The combustible and zero (or empty) waste matrices ${ }^{239} \mathrm{Pu}$ gram amounts are 6.16 and $92.27 \mathrm{~g}$, respectively. The test results are summarized in the PDP report for Cycle 4.[PDP97c ] The total alpha-Ci \%R and \%RSD are 99.06 and 1.54 and 109.83 and 2.95 for the empty and combustible drums, respectively. The average of the PDP alpha-Ci $\% \mathrm{R}$ and $\%$ RSD is 104 and 2.2, respectively. The weighted alpha-Ciaverage of the three tests (CEP, RCI and PDP) \%R and \%RSD is 119 and 3.3, respectively.

Several different radioactive isotopes within actual waste drums have been detected while WIT was at LLNL, RFETS, INEEL and NTS. A summary of these isotopes is presented in Table III.C-3.

Table III.C-3 Radionuclides identified in waste by BIR WIT/A\&PCT.

\begin{tabular}{|c|c|c|}
\hline U \& TRU & $\begin{array}{c}\text { Other } \\
\text { Actinides }^{a}\end{array}$ & $\begin{array}{c}\text { Other } \\
\text { Radionuclides-source }\end{array}$ \\
\hline${ }^{235} \mathrm{U}$ & ${ }^{208} \mathrm{Tl}$ & ${ }^{22} \mathrm{Na}-\mathrm{F}(\alpha, \mathrm{p})$ \\
\hline${ }^{238} \mathrm{U}$ & ${ }^{219} \mathrm{Rn}$ & ${ }^{40} \mathrm{~K}-$ bckgd. \\
\hline${ }^{237} \mathrm{~Np}^{\mathrm{a}}$ & ${ }^{223} \mathrm{Ra}$ & ${ }^{60} \mathrm{Co}-f p^{\mathrm{c}}$ \\
\hline${ }^{238} \mathrm{Pu}^{\mathrm{b}}$ & ${ }^{227} \mathrm{Th}$ & ${ }^{137} \mathrm{Cs}-f p$ \\
\hline${ }^{239} \mathrm{Pu}$ & ${ }^{243} \mathrm{Cm}$ & ${ }^{211} \mathrm{~Pb}-\mathrm{bckgd}$. \\
\hline${ }^{240} \mathrm{Pu}$ & ${ }^{249} \mathrm{Cf}$ & ${ }^{214} \mathrm{Bi}-\mathrm{bckgd}$. \\
\hline${ }^{241} \mathrm{Pu}$ & & \\
\hline${ }^{241} \mathrm{~A} \mathrm{~m}$ & & \\
\hline
\end{tabular}

Notes: a. Primarily identified in LLNL waste drums at NTS; some were identified in drums at LLNL, RFETS and INEEL.

b. ${ }^{238} \mathrm{Pu}$ can be seen in samples enriched in this isotopc, but most waste drums contain WG Pu and its percentage abundance is too small for its gamma-rays to be detected.

c. $f p$ is fission product.

\section{III.D Summary of A\&PCT Performance}

A summary of IMPACT's and WIT/A\&PCT's performance results in terms of ${ }^{234} \mathrm{Pu}$ mass for the \%R and \%RSD parameters are shown in Table III.D-1 and Figures III.D-1 and III.D-2. Here, we only report the ${ }^{239} \mathrm{Pu}$ mass results for surrogate drums since the ${ }^{239} \mathrm{Pu}$ mass within these surrogate drums is precisely known and NIST traceable. The actual CEP and RCI waste drum data is excluded from the table and figures simply because there is uncertainty associated with their ${ }^{233} \mathrm{Pu}$ mass. All reported performance measure values 
are based on the IMPACT and WIT/A\&PCT data and UCSF-MLEM image reconstruction and assay procedure using the $414-\mathrm{keV}$ peak of ${ }^{239} \mathrm{Pu}$. Using all values reported in Table III.D-1 the average \%R and \%RSD for IMPACT is 69 and 0.7 , respectively and WIT/A\&PCT is 132 and 2.3 , respectively. The WIT/A\&PCT \%RSD was determined only using the CEP and PDP data since they were the only data with multiple replicates.

The $\% \mathrm{R}$ versus increasing gram amounts of ${ }^{239} \mathrm{Pu}$ for the performance test demonstrate that the IMPACT UCSF-MLEM assay results are $\sim 30 \%$ low (Figure III.D-1). As mentioned earlier we have reduced this negative bias to approximately $-10 \%$ using the APCT-CCG code (see Figure III.B.3-9). Three of the seven WIT/A\&PCT scanner UCSF-MLEM data are within $+10 \%$ of the true value. However, there is a grouping near a \%R of 145 and one with a \%R of 175. These large positive biases are most likely due to insufficient counts resulting in a large positive bias as expected. The $0.7 \%$ RSD for IMPACT is lower than that for WIT /A\&PCT, 2.3-this is only for the CEP and PDP data $\%$ RSD, which are the only true measures of \%RSD for WIT/A\&PCT.

Table III.D-1: IMPACT and WIT UCSF-MLEM blind test results for surrogate drums.

\begin{tabular}{|l|c|c|c|c|}
\hline \multicolumn{3}{|c|}{ Drum } & \multicolumn{2}{c|}{ Measurement } \\
$\begin{array}{c}\text { Test } \\
\text { System }\end{array}$ & $\begin{array}{c}\text { Rep. \# } \\
(\mathbf{g r a m s}\end{array}$ & $\begin{array}{c}\text { \%ample ID } \\
{ }^{23} \mathbf{P u}\end{array}$ & $\begin{array}{c}\text { \%R for } \\
\text { (Matrix) }\end{array}$ & $\begin{array}{c}\text { \%RSD } \\
\text { (Mu mass } \\
\text { for }{ }^{239} \mathbf{P u} \\
\text { mass }\end{array}$ \\
\hline $\begin{array}{l}\text { IMPACT } \\
\text { PDP-3 }\end{array}$ & $\begin{array}{c}6 \\
(66.87)\end{array}$ & $\begin{array}{c}\text { Drum 003 } \\
\text { (Comb.) }\end{array}$ & 66.06 & 0.58 \\
\hline $\begin{array}{l}\text { IMPACT } \\
\text { PDP-3 }\end{array}$ & $\begin{array}{c}6 \\
(92.11)\end{array}$ & $\begin{array}{c}\text { Drum 004 } \\
\text { (Glass) }\end{array}$ & 71.21 & 0.84 \\
\hline $\begin{array}{l}\text { WIT } \\
\text { RCI }\end{array}$ & $\begin{array}{c}1 \\
(2.22)\end{array}$ & $\begin{array}{c}\text { 1-SG } \\
\text { (Glass) }\end{array}$ & 139.7 & $2.8^{3}$ \\
\hline $\begin{array}{l}\text { WIT } \\
\text { RCI }\end{array}$ & $\begin{array}{c}1 \\
(0.933)\end{array}$ & $\begin{array}{c}\text { 2-SG } \\
\text { (Comb.) }\end{array}$ & 149.9 & $2.7^{4}$ \\
\hline $\begin{array}{l}\text { WIT } \\
\text { RCI }\end{array}$ & $\begin{array}{c}1 \\
(0.747)\end{array}$ & $\begin{array}{c}\text { 3-SG } \\
\text { (Metals) }\end{array}$ & 174.0 & $2.7^{4}$ \\
\hline \hline $\begin{array}{l}\text { WIT } \\
\text { PDP-4 }\end{array}$ & $\begin{array}{c}6 \\
(6.16)\end{array}$ & $\begin{array}{c}\text { Drum 003 } \\
\text { (Comb.) }\end{array}$ & 109.9 & 2.92 \\
\hline $\begin{array}{l}\text { WIT } \\
\text { PDP-4 }\end{array}$ & $\begin{array}{c}6 \\
(92.27)\end{array}$ & $\begin{array}{c}\text { Drum 001 } \\
\text { (Zero) }\end{array}$ & 98.73 & 2.14 \\
\hline \hline $\begin{array}{l}\text { WIT } \\
\text { CEP }\end{array}$ & $\begin{array}{c}8 \\
(46.0)\end{array}$ & $\begin{array}{c}\text { SG-6 } \\
\text { (MSE Salt) }\end{array}$ & 103.53 & 1.54 \\
\hline $\begin{array}{l}\text { WIT } \\
\text { CEP }\end{array}$ & $\begin{array}{c}8 \\
(0.961)\end{array}$ & $\begin{array}{c}\text { SG-9 } \\
\text { (Raschig) }\end{array}$ & 145.96 & 2.67 \\
\hline
\end{tabular}

Note: 1. The RCI and CEP were both scored by LMITCO INEEL and the PDP was scored by DOE-CAO [PDP97b, PDP97c]. All data include 6 (PDP) to 8 (RCI \& CEP) replicates per drum for these tests. All WIT test measurements were performed at INEEL.

2. Number of replicate scans on top in a parentheses actual ${ }^{239} \mathrm{Pu}$ content in grams.

3. Determined from the IMPACT 15 replicate QAO test at 3.6 grams of WG Pu.

4. Acquired from the CEP test sample SG-9 at $0.961{ }^{239} \mathrm{Pu}$. 


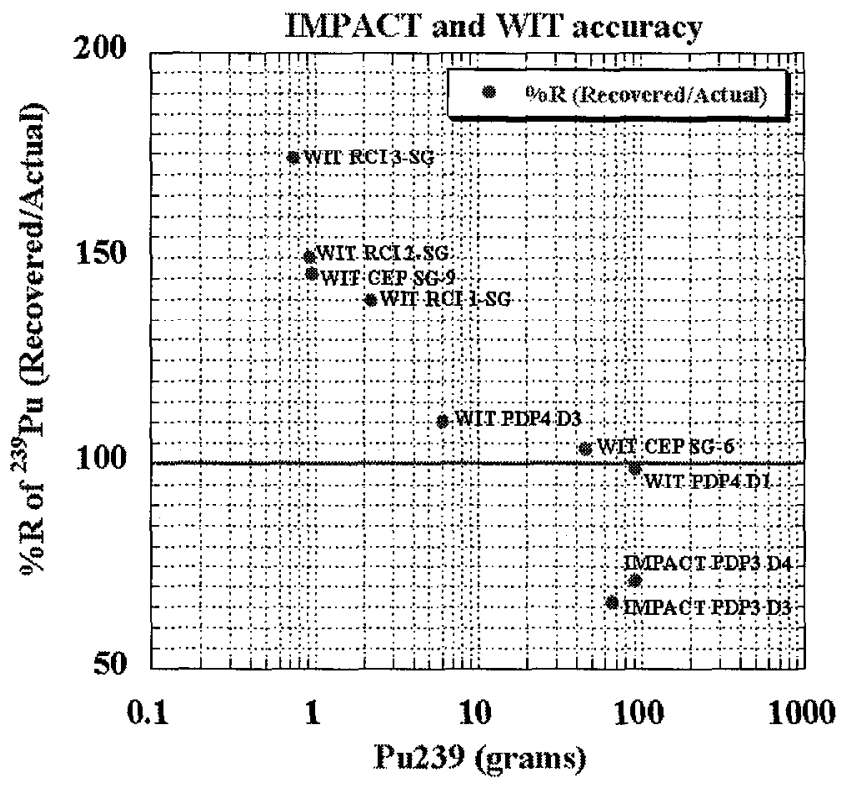

Figure III.D-1 Percent recovery versus increasing grams of ${ }^{239} \mathrm{Pu}$ for PDP, RCI, and CEP performance tests to demonstrate total bias of IMPACT and WIT/A\&PCT.

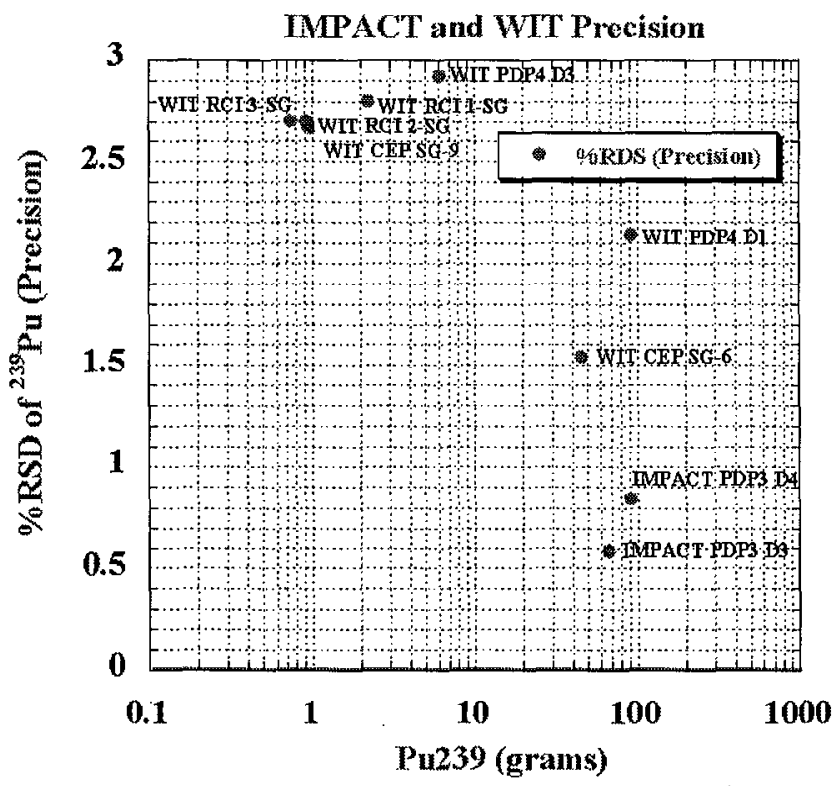

Figure III.D-2 Percent relative standard deviation versus increasing grams of ${ }^{239} \mathrm{Pu}$ for PDP, RCI, and CEP performance tests to demonstrate precision of IMPACT and WIT/A\&PCT. 


\section{Implementation/Deployment Status}

LLNL has developed two A\&PCT scanners. The first is developed, maintained and operated by the Nondestructive and Materials Evaluation Section within the Engineering Directorate at LLNL; the second is owned and operated by BIR, Inc. and is installed within a mobile trailer. These systems have been used to assay a wide range of radioactive transuranic waste. These waste forms contained ${ }^{239} \mathrm{Pu}$ mass amounts ranging from $\sim 0.5$ through $\sim 100 \mathrm{~g}$. The ${ }^{239} \mathrm{Pu}$ was contained within several different waste matrices typical of the DOE inventory, e.g., combustibles, metals, and sludge.

The preliminary performance of LLNL's IMPACT scanner using the UCSFMLEM code for surrogate waste drums was determined to have a total bias in terms of \%R of $\sim 70$ with a \%RSD of 0.7 for combustible and glass matrices. It is important to note as discussed in Section II.B.3.3 that the newly developed image reconstruction and assay code APCT-CCG shows a \%R of $\sim 90$ a factor of three improvement in the negative bias. The performance of the WIT/A\&PCT scanner using the UCSF-MLEM code for surrogate waste drums was determined to have a bias in \%R of 132 with a \%RSD of 2.3 for a broad range of matrices. Additional R\&D is required to better understand IMPACT's negative $10 \%$ bias for good statistical data sets and to reduce the high-positive bias observed in both systems for poor statistical data.

The WIT/A\&PCT scanners total bias in \%R for drums with ${ }^{239} \mathrm{Pu}$ gram amounts of greater than 45 is 101 while that of IMPACT's using UCSF-MLEM is only 70 . The only difference between the two scanners is the septa included within the WIT/A\&PCT detector aperture collimator. The new image reconstruction and assay codes are near completion and the APCT-CCG code appears to reduce both the positive and negative biases. LLNL is automating the isotopic analysis, determining systematic uncertainties and MDCs, and validating performance for the four QAPP activity ranges. The total measurement uncertainty has been addressed and is being evaluated for WIT/A\&PCT.

\section{A Demonstrated Capabilities}

Data acquired to date indicates that the A\&PCT technology has demonstrated that it can localize, quantify and identify TRU isotopes; and that this quantification can be done independent of the type of waste matrix. This technology has the capability to determine radioactive material quantities on a voxel basis, only six-ten thousandths of a 208-liter drum. This helps remove nearly $100 \%$ of the bias that can arise from nonuniform radioactive material and waste matrix distributions. Most of the other NDA technologies use other techniques with much greater uncertainty to account for the spatial bias effects.

Further, we have demonstrated that this technology has been successfully transferred from the R\&D realm to an industrial partner. They have commercialized this technology and have demonstrated that this technology 
can be incorporated into a mobile laboratory. Through the three test programs CEP, RCI and PDP the mobile technology is viable in terms of complying with NTP QAPP requirements. Furthermore, the three NDE and one NDA technologies incorporated into this mobile laboratory are fully automated and are operated by trained technicians. Both the laboratory-based A\&PCT system (IMPACT) and the mobile-based A\&PCT system (WIT/A\&PCT) participated and passed all of the performance (blind) tests entered to date.

Again it is important to note that compliance has been demonstrated using the simple calibration method of the A\&PCT technology. This calibration method consists of the measurement of only one NIST traceable point source at a known distance and it does not require the use of a suite of calibration matrices and assorted radioactive working reference materials (WRMs). The entire calibration procedure takes on the order of two hours, as opposed to the many months of resource intensive calibration procedures and expensive apparatus required by other NDA technologies.

The A\&PCT technology provides measurement times that can be scaled to the amount of activity and to the drum fill-height found within a drum by the fast survey CGS mode. The CGS mode allows for a quick scan of the waste drum to determine the approximate radioactive material location(s) and mass amounts. This CGS data allows A\&PCT scan times to be customized to acquire sufficient statistics and to ensure that accurate measurements are made. This feature provides for optimal count times to obtain desired accuracy and precision within the shortest period of time.

\section{IV.B Limitations}

Each specific NDE and NDA technology has intrinsic limitations that derive from the physical basis of their measurement process. The A\&PCT technology is a gamma-ray based tomographic technique, hence it cannot quantify isotopes that do not emit gamma-rays. The CAO requirement to report ${ }^{90} \mathrm{Sr}$, a pure beta emitter, cannot be measured by this technology or any other commonly used NDA technology.

Another unfortunate limitation of all gamma-ray based NDA technologies arises from the very low branching ratios characteristic of many gamma-ray transitions found in transuranic isotopes of interest to NTP. As an example, the isotope of most interest to $\mathrm{NTP},{ }^{239} \mathrm{Pu}$, has a branching ratio of only $10^{-5}$ for its $414-\mathrm{keV}$ gamma-ray transition. Although the $100-\mathrm{keV}$ gamma-ray transitions found in many transuranic isotopes of interest do have larger branching ratios, they are more highly attenuated and result in poorer statistical data for a fixed integration time. Long transuranic half lives and low gamma-ray transition branching ratios are the primary reasons that the A\&PCT technology is stated to be "physics limited." We cannot make a long half life transuranic isotope decay faster nor increase its branching ratio to gives us a counting rate higher than nature allows. 
The physics limited aspects of long-half lives and small branching ratios and the tomographic measurement protocol all contribute to a reduction in waste drum throughput. This situation has the greatest impact on those drums having low transuranic alpha-activity amounts $(0.002-0.02 \alpha-\mathrm{Ci})$ and those near the MDC limit. For low activity drums measurement times must be longer to obtain better counting statistics to provide more accurate assays. The best we can do to solve the physics limited and throughput limitations is to increase the number of detectors and thereby reduce measurement time (or increase our sensitivity) while still preserving the principles of tomographic imaging and assay. We are currently upgrading the technology to a multiple detector system to address this limitation (see section IV.C.1).

In certain circumstances, there may be a large increase in throughput gained by implementing a scan geometry that differs from traditional computed tomography. For example, sludge drums are a challenge to gammaray-based assay systems, particularly in the transmission mode. In the passive mode, the internal isotopic sources only need to penetrate half the diameter of the drum to be detected; however, in the active/transmission mode, near the center of the drum the transmission gamma rays need to penetrate the entire diameter of the drum before they are detected. Acquiring active data on sludge drums can be a time consuming effort because each ray sum requires a long integration time to collect the necessary statistics for image reconstruction. Increasing the active source strength is one method to improve this situation; however, there are problems associated with license thresholds, safety of personnel, size and weight of the required shielding, and saturation of the detectors when the sludge is not in the field of view (i.e., the drum may not be filled all the way to the top with sludge).

Another limitation may be related to the A\&PCT technology's ability to correct for large transuranic lumps (or clumps) of material greater than several millimeters in extent. For example, 1-mm diameter-sized spheres of a TRU isotope are preferentially self attenuating of their own lower-energy gamma emissions. Larger lumps may not be properly imaged by the ACT transmission method since they occupy a very small fraction of the voxel volume. Thus, the proper quantification of such lumps of TRU material would derive by combining results from NDE digital radiography and/or tomography with the NDA A\&PCT results, and perhaps even with passive NDA neutron based measurements. This is just one example where the fusion of data from one or several NDE modes with data from neutron- and gamma-ray based NDA systems may provide more accurate waste drum characterization.

\section{IV.C Future Development Activities}

There are six areas associated with the A\&PCT technology that are currently being developed or should be further developed. They include multiple detectors, "smart" scanning techniques to shorten drum scan times, further optimization of the automated isotopic analysis program, continued testing of lump correction techniques, further investigation into the apparent bias as a 
function of mass, and the fusion of other NDE and NDA modalities that could lead to more accurate assay values and more complete waste characterizations.

\section{IV.C.1 Multiple Detector Development}

As mentioned in the previous section, one limiting restriction to the A\&PCT technology is the relatively long scan time required to assay a drum. In part, long scan times are due to the use of only a single HPGe detector for recording data, and the overhead (time spent) associated with discrete movement of the drum when no data is acquired. We are working on methods to reduce data acquisition scan times in the ACT and PCT modes without compromising the accuracy of the assay.[ROB97]

We are working with BIR to upgrade the WIT/A\&PCT scanner to incorporate multiple detectors and to include a continuous motion scanning mode. Figure IV.C.1-1 shows the expected speed up of the new scanner (red and green lines), and is compared to the current single-detector A\&PCT scanner (blue line). The ACT and PCT ray sum integration times used for a drum assay are dependent on the waste matrix gamma-ray attenuation and the activity level of the transuranic radioisotopes. Depending on the ray sum integration times used, the new A\&PCT system design should reduce the counting time by a factor of 10 for the long integration times currently required to assay low-activity waste and to perhaps as much as a factor of 40 for the highest levels of radioactive waste.

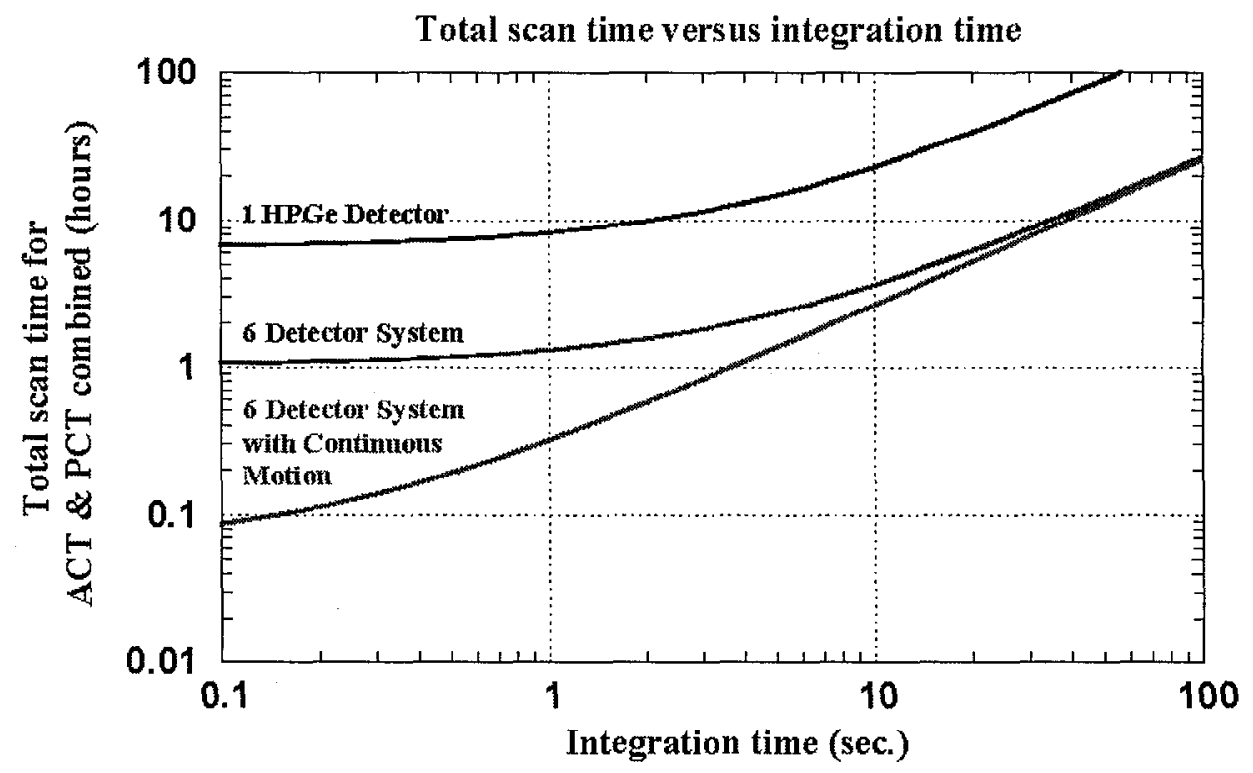

Figure IV.C.1-1: The total active and passive assay scan times required for various A\&PCT scanner configurations plotted as a function of ray-sum integration time. The ray-sum integration times shown are the same for both active and passive modes. 


\section{IV.C.2 Smart Scan Techniques}

There are methods that will increase the throughput of the A\&PCT technology. One solution may be to develop a "smart" scanning scenario for dense $\left(>0.7 \mathrm{~g} / \mathrm{cm}^{3}\right)$ waste drums. If they are found to be relatively homogeneous when evaluated by an NDE radiographic or transmission CT system, then a single ray sum of the drum and matrix is all that is required to computationally construct the active volume image. This smart scanning method would decrease the active counting time by a factor of up to 1000 . There may be other smart scanning scenarios that are waste matrix dependent. These could be studied and also used to increase waste drum throughput.

\section{IV.C.3 Automated Isotopic Analysis}

We have developed an automated isotopic analysis program for any TRU waste stream. This program is designed to be flexible enough so that it can be tailored to variable waste streams. Our experience in analyzing TRU waste isotopics will improve over time and reveal changes and additions that could even improve the isotopic analysis code. These enhancements may include the addition of isotopes that were not initially expected in the TRU waste streams, and optimization for isotopes that occur more than originally expected. Incorporating these and other future experiences will lead to a more robust automated operation of the code.

\section{IV.C.4 Lump Corrections}

Lump correction is required when the size of the radioactive material or lump being assayed is massive enough to self-attenuate its own gamma-ray emissions. Although we have developed a method to correct for the selfattenuation expected from certain size lumps of $\mathrm{Pu}$, it may be possible to improve this technique by other methods. Just recently PDP standards that exhibit self-attenuation problems have become available. These PDP sources are well characterized and should reveal what methods show the most promise for solving this problem. However, these sources should also be used to evaluate and optimize current methods that show promise for solving the lump problem. Further work may be necessary in this area to develop new methods or improve existing methods for lump corrections.

One possible solution to this problem is to use a high-spatial resolution transmission computed tomography volume image fused with the A\&PCT image to identify where TRU lumps are located. If the TRU lumps can be identified within the transmission CT image, a segmentation process can be performed on the volume to extract the objects. Segmentation is a process that is used to remove an object of interest from a CT volume image based on pixel values and connectivity.[BOS96] Once the TRU lump is computationally removed, the volume can be determined from the dimensionally correct pixel number that represents the object. If this method is feasible, it could be implemented on a system like the WIT trailer because it contains a transmission CT scanner that has adequate spatial resolution. 


\section{IV.C.5 Assay Bias Evaluation}

Data acquired to date indicates an apparent bias as a function of mass. We have investigated and partially addressed this situation. For example, the negative $30 \%$ IMPACT bias obtained using the UCSF-MLEM code for assays was reduced to only a negative $10 \%$ bias using the APCT-CCG code on the same data. We also have reduced the positive bias but not significantly. Thus, we are not thoroughly convinced that this bias is completely resolved; therefore, further evaluation is needed.

\section{IV.C.6 Data Fusion}

Data fusion is the process of integrating the results from both NDE and NDA characterization techniques to achieve a more accurate assay or to increase the confidence of an assay. The potential of this integration has yet to be realized. The integration of A\&PCT quantitative data and NDE highspatial resolution data may solve problems related to lump corrections as mentioned above. Also, NDE radiographs or CT images can be used to determine information about the homogeneity of a drum that in turn can be used to determine the optimum A\&PCT scan geometry that should be used to obtain maximum throughput. The integration of other NDA assay modes with A\&PCT data sets will provide increased accuracy and confidence.

\section{Acknowledgments}

We thank Steve Azevedo, DeLynn Clark, Gene Ford, Dennis Goodman, Erik Johansson, Eric Keto and Sailes Sengupta at LLNL for their many contributions during various development stages of this A\&PCT technology. We also thank Mr. Richard Bernardi and his staff at BIR for their support of our R\&D effort, for their help in the technology transfer of A\&PCT to BIR, and for allowing us to use WIT test results in this report. Part of this work (BIR) was funded by several Programmatic Research and Development Agreements (PRDA) from the Morgantown, WV office of DOE's Environmental Restoration and Waste Management Program (EM-50). Another part (LLNL) was funded by DOE's Mixed Waste Focus Area (MWFA) and by the Federal Energy Technology Center's Office of Science and Technology in DOE's Environmental Restoration and Waste Management Program; and it was performed under the auspices of the U.S. Department of Energy by the Lawrence Livermore National Laboratory under Contract W-7405-Eng-48. One of us (GKB) was supported by funding from the DOE's MWFA and from the Characterization Monitoring and Sensor Technology (CMST) program of DOE's EM-50. 


\section{References}

[BEC97] G.K. Becker, and J.C. Determan, "Application of Expert System Technology To Nondestructive Waste Assay - Initial Prototype Model," Proceedings of the 5th Nondestructive Assay and Nondestructive Examination Waste Characterization Conference, Salt Lake City, Utah, Jan. 14-16, 1997, INEL CONF-970126, p. 455

[BER95] Richard T. Bernardi and Harry E. Martz, Jr., "Nuclear Waste Drum Characterization with $2 \mathrm{MeV}$ X-ray and Gamma-ray Tomography," Proceedings of the SPIE's 1995 International Symposium on Optical. Science, Engineering, and Instrumentation (Vol. 2519), San Diego, CA, July 13-14, 1995.

[BER97] R. T. Bernardi, "Field Test Results for Radioactive Waste Drum Characterization with Waste Inspection Tomography (WIT)," Proceedings of the 5th Nondestructive Assay and Nondestructive Examination Waste Characterization Conference, Salt Lake City, Utah, Jan. 14-16, 1997, INEL CONF-970126, p. 107.

[BOS96] "Application of 3D X-Ray CT Data Sets to Finite Element Analysis," P-L Bossart, H. E. Martz, H. R. Brand, K. Hollerbach, Review of Progress in Quantitative Nondestructive Evaluation, D. O. Thompson and D. E. Chimenti, Eds. (Plenum Press, New York, 1996) 15 pp. 489-496.

[BRO95] J.K. Brown, K. Kalki, J.A. Heanue, and B.H. Hasegawa, "Quantitative SPECT Reconstruction Using Multiray Projection Integrators," Conference Record 1995 IEEE Nuclear Science Symposium and Medical Imaging Conference Vol. 2(1995)1272-1276.

[BUD79] T. F. Budinger, G. T. Gullberg, R. H. Huesman, “Emission Computed Tomography," in Image Reconstruction from Projections Implementation and Applications, G.T. Herman, Ed. (Springer Verlag, New York, 1979), p. 147.

[COU98] C. Robert-Coutant, V. Moulin, R. Sauze, and Ph. Rizo, "Estimation of the Matrix Attenuation in Heterogeneous Radioactive Waste Drums Using Dual-Energy Computed Tomography," Presented at 1998 Symposium on Radiation Measurements and Applications, May 11-14, 1998, Ann Arbor, MI.

[DEB88] K. Debretin and R. G. Helmer, Gamma- and X-Ray Spectrometry with Semiconductor Detectors, (Elsevier, New York, NY, 1988).

[DEC96] Daniel J. Decman, Harry E. Martz, G. Patrick Roberson, and Erik Johansson, "NDA via Gamma-ray Active and Passive Computed Tomography," Mixed Waste Focus Area Final Report, Lawrence Livermore National Laboratory, Livermore, Calif., UCRL-ID125303, November 1996. 
[DUN97] Dung, T. Q., "Calculation of the systematic error and correction factor in gamma waste assay system," Ann. Nucl. Energy, Vol. 24 No. 1(1997) 33-47.

[EST94] R.J. Estep, T.H. Prettyman, and G. A. Sheppard, "Tomographic Gamma Scanning to Assay Heterogeneous Radioactive Waste," Nucl. Sci. Eng. Vol. 118 (1994) p. 145-152.

[EST97] R. J. Estep and Sheila Melton, "Using NaI Detectors for Tomographic Gamma Scanning," Proceedings of the 5th Nondestructive Assay and Nondestructive Examination Waste Characterization Conference, Salt Lake City, Utah, Jan. 14-16, 1997, INEL CONF-970126, p. 395.

[GOO93] D. M. Goodman, E. M. Johansson, and T. W. Lawrence, "On Applying the Conjugate Gradient Algorithm to Image Processing Problems," Chapter 11 in Multivariate Analysis: Future Directions, C. R. Rao, Editor, Elsevier Science Publishers, 1993.

[GOO93a] D. M. Goodman, T. W. Lawrence, E. M. Johansson, and J. P. Fitch, "Bispectral Speckle Interferometry to Reconstruct Extended Objects from Turbulence-Degraded Telescope Images," Chapter 13 in Handbook of Statistics, Vol. 10: Signal Processing and its Applications, N. K. Bose and C. R. Rao, Editors, North Holland, 1993.

[GO097] D. M., Goodman, "Maximum Likelihood Estimation with Poisson (Counting) Statistics for Waste Drum Inspection," UCRL-ID-127361, Lawrence Livermore National Laboratory, Livermore, CA May 1997.

[GOT91] H. Gotoh, Japan Atomic Energy Research Institute, Tokai-Mura, Naka-Gun, Ibaraki-ken 319-11 Japan, private communication (1991).

[GRE97] J. Gregor and D. C. Hensley, "Gamma-Ray Imaging of the Quinby Sources," Proceedings of the 5th Nondestructive Assay and Nondestructive Examination Waste Characterization Conference, Salt Lake City, Utah, Jan. 14-16, 1997, INEL CONF-970126, p. 361.

[HAD95] W. S. Haddad, J. E. Trebes, D. M. Goodman, H. R. Lee, I. McNulty, E. H. Anderson, A. O. Zalensky, Ultra High Resolution Soft X-Ray Tomography, Proc. SPIE Conf., San Diego, July, 1995.

[IIEN97] D. C. Hensley, "An Overview of the ORNL-NFS Intercomparison," Proceedings of the 5th Nondestructive Assay and Nondestructive Examination Waste Characterization Conference, Salt Lake City, Utah, Jan. 14-16, 1997, INEL CONF-970126, p. 93.

[HOG97] R. A. Hogle, P Miller, and R. L. Bramblett, "APNEA List Mode Data Acquisition and Real-time Event Processing," Proceedings of the 5th Nondestructive Assay and Nondestructive Examination Waste Characterization Conference, Salt Lake City, Utah, Jan. 14-16, 1997, INEL CONF-970126, p. 183. 
[HOL97] Charles L. Hollas, Gaetano Arnone, Glenn Brunson, and Kenneth Coop, "Matrix Effects Corrections in DDT Assay of ${ }^{239}$ PU With the CTEN Instrument," Proceedings of the 5th Nondestructive Assay and Nondestructive Examination Waste Characterization Conference, Salt Lake City, Utah, Jan. 14-16, 1997, INEL CONF970126.

[HUE77] R. H. Huesman, G. T. Gullberg, W. L. Greenberg, and T. F. Budinger, RECLBL Library Users Manual, Lawrence Berkeley Laboratory, Berkeley, CA, Pub 214 (1977).

[JAC98] Jessie A. Jackson, Dennis Goodman, G. Patrick Roberson, and Harry E. Martz, "An Active and Passive Computed Tomography Algorithm with a Constrained Conjugate Gradient Solution," submitted to Proceedings of the 6th Nondestructive Assay Waste Characterization Conference, Salt Lake, Utah, November 17-19, 1998.

[KAW90] S. Kawasaki, M. Kondo, S. Izumi, and M. Kikuchi, "Radioactivity Measurement of Drum Package Waste by a ComputedTomography Technique," Appl. Radiat. Isotopes Vol. 41(1990)983.

[KET95] Eric Keto, Steve Azevedo, Pat Roberson, Dan Decman, Harry Martz and Erik Johansson, "Spatial Resolution Versus Signal to Noise in Quantitative Tomography" Proceedings of the 4th Nondestructive Assay \& Nondestructive Examination Waste Characterization Conference, Salt Lake, Utah, October 24-26, 1995, pp. 405-420.

[KOL94] J. Kolman, W. S. Haddad, D. M. Goodman, and K. A. Nugent, "Application of a Constrained Optimization Algorithm to Limited View Tomography," Proc. SPIE Conf., San Diego, July, 1994.

[LEV95] Lévai, F., "Evaluation of the Waste Assay System for compacted 200-L waste drummes used at Paks Power plant and its development," Interim Report, Technical University, Budapest, Institute of Nuclear Techniques, November 1995.

[LEV95a] Lévai, F., Nagy, Z. S. and Dung, T. Q., "Low Resolution Combined Emission-Transmission Imaging Techniques for Matrix Characterization and Assay of Waste," Proceedings of the 17th ESARDA Symposium, Aachen, May (1995) 319-323.

[MAR91] H. E. Martz, G. P. Roberson, D. J. Schneberk, and S. G. Azevedo, "Nuclear-Spectroscopy-Based, First-Generation, Computerized Tomography Scanners", IEEE Trans. Nucl. Sci. Vol. 38(1991) 623.

[MAR91a] H. E. Martz, S. G. Azevedo, G. P. Roberson, D. J. Schneberk, Z. M. Koenig, and D. C. Camp, "Considerations for an Active and Passive CT Scanner to Assay Nuclear Waste Drums," ASNT's Industrial Computed Tomography Conference II, Topical Conference Paper Summaries, May 20-24, San Diego, CA, (1991)143. 
[MAR92] H. E. Martz, G. P Roberson, C. Robert-Coutant, and D. C. Camp, "Experimental A\&PCT Research and Development Efforts to Characterize Mixed Waste Forms," Proceedings of the Transuranic Waste Characterization Conference, Idaho State University, Pocatello, Idaho, August 10-12, 1992.

[MAR95] Harry E. Martz, Daniel J. Decman, G. Patrick Roberson, David C. Camp, and Richard T. Bernardi, "Radioactive Waste Realities as Revealed by X- and Gamma-ray Measurements," American Chemical Society 1995 Extended Abstracts for the Special Symposium on Emerging Technologies in Hazardous Waste Management VII, Atlanta, GA, September 17-20, 1995, pp. 889-894.

[MAZ97] C. J. Marcinkiewicz, M. J. Connolly and G. K. Becker, "Performance in the WIPP Nondestructive Assay Performance Demonstration Program," Proceedings of the 5th Nondestructive Assay and Nondestructive Examination Waste Characterization Conference, Salt Lake City, Utah, Jan. 14-16, 1997, INEL CONF-970126, p. 175.

[MIL95] T. E. Milner, D. M. Goodman, B. S. Tanenbaum, and J. S. Nelson, "Depth Profiling of Laser-Heated Cromophores in Biological Tissues by Pulsed Photothermal Radiometry," Journal of the Optical Society of America-A, Vol. 12 No. 7, July 1995.

[MIL96a] T. E. Milner, D. J. Smithies, D. M. Goodman, A. Lau, and J. S. Nelson, "Depth Determination of Cromophores in Human Skin by Pulsed Photothermal Radiometry," Applied Optics, June, 1996.

[MIL96b] T. E. Milner, D. M. Goodman, B. S. Tannenbaum. B. Anvari, and J. S. Nelson, "Noncontact Determination of Thermal Diffusivity in Biomaterials Using Infrared Imaging Radiometry," J. Biomedical Optics, Vol. 1, No. 1, Jan. 1996.

[MIL96] T. E. Milner, D. M. Goodman, B. S. Tanenbaum, B. Anvari, L. O. Svasand, and J. S. Nelson, "Imaging of Laser Heated Subsurface Chromophores in Biological Materials: Determination of Lateral Physical Dimensions," Phys. in Med. and Bio., Vol. 41, 1996.

[NDA] See Proceedings of the 4th Nondestructive Assay and Nondestructive Examination Waste Characterization Conference, Salt Lake City, Utah, Oct. 24-26, 1995, INEL CONF-951091; Proceedings of the 5th Nondestructive Assay and Nondestructive Examination Waste Characterization Conference, Salt Lake City, Utah, Jan. 14-16, 1997, INEL CONF-970126; and Proceedings of the 6th Nondestructive Assay Waste Characterization Conference, Salt Lake, Utah, November 17-19, 1998 in press.

[PDP97] "Performance Demonstration Program Plan for Non-Destructive Assay for the TRU Waste Characterization Program," U.S. 
Department of Energy, Carlsbad Area Office, National TRU Program Office, CAO-94-1045, Revision 1, May 1997.

[PDP97a] "Performance Demonstration Program Plan for Non-Destructive Assay for the TRU Waste Characterization Program, Scoring Report," November 1996 Distribution prepared by Lockheed Martin Idaho Technologies Company, Idaho Falls, ID, for U.S. Department of Energy, Carlsbad Area Office, National TRU Program Office, January 1997.

[PDP97b] "Performance Demonstration Program Plan for Non-Destructive Assay for the TRU Waste Characterization Program, Scoring Report," May 1997 Distribution prepared by Lockheed Martin Idaho Technologies Company, Idaho Falls, ID, for U.S. Department of Energy, Carlsbad Area Office, National TRU Program Office, July 1997.

[PDP97c] "Performance Demonstration Program Plan for Non-Destructive Assay for the TRU Waste Characterization Program, Scoring Report," September 1997 Distribution prepared by Lockheed Martin Idaho Technologies Company, Idaho Falls, ID, for U.S. Department of Energy, Carlsbad Area Office, National TRU Program Office, November 1997.

[PIC98] M.M. Pickrell, D.J. Mercer, and T.J. Sharpe, “A Technique for Combining Neutron and Gamma-ray Data into a Single Assay Value," presented at the INMM 39th Annual Meeting, Naples, FL, July 26-30, 1998 and LA-UR-98-3203, Los Alamos National Laboratory, Los Alamos, NM.

[PRE95] T.H. Prettyman, S.E. Betts, D.P. Taggart, R.J. Estep, N.J. Nicholas, M.C. Lucas, and R.A. Harlan, "Field Experience with a Mobile Tomographic Nondestructive Assay System," LA-UR-95-3501, Los Alamos National Laboratory, Los Alamos, NM, October 1995.

[QAP96] "Transuranic Waste Characterization Quality Assurance Program Plan," U.S. Department of Energy, Carlsbad Area Office, National TRU Program Office, CAO-94-1010, Interim Change, November 15, 1996.

[REI92] P. Reimers, "Quality Assurance of Radioactive Waste Packages by Computerized Tomography, Task 3, Characterization of Radioactive Waste Forms; A Series of Final Reports (1985-89) - No. 37," Nuclear Science and Technology, EUR 13879 EN, Commission of the European Communities, Luxembourg, 1992.

[ROB91] G.P. Roberson, H.E. Martz, D.J. Schneberk, and C.L. Logan, "NuclearSpectroscopy Computerized Tomography Scanners," 1991 ASNT Spring Conference, March 18-22, Oakland, California, 107, (1991).

[ROB94] G. P. Roberson, H. E. Martz, D. J. Decman, D. C. Camp, S. G. Azevedo and E. R. Keto, "Characterization of Waste Drums Using 
Nonintrusive Active and Passive Computed Tomography," Proceedings of the 4th Nondestructize Assay and Nondestructive. Examination Waste Characterization Conference, Idaho State University, Pocatello, Idaho, February 14-16, 1994 pp. 261-294.

[ROB95] G.P. Roberson, D. J. Decman, H. E. Martz, E. R. Keto and E. M. Johansson, "Nondestructive Assay of TRU Waste Using Gammaray Active and Passive Computed Tomography," Proceedings of the 4th Nondestructive Assay and Nondestructive Examination Waste Characterization Conference, Salt Lake City, Utah, October 24-26, (1995) 73-84.

[ROB97] G.P. Roberson, H. E. Martz, D. C. Camp, D. J. Decman and E. M. Johansson, "Preliminary A\&PCT Multiple Detector Design, Upgrade of a Single HPGe detector A\&PCT System to Multiple Detectors," Lawrence Livermore National Laboratory, Livermore, Calif., UCRL-ID-128052, June 1997.

[ROB98] G. Patrick Roberson, Harry E. Martz, Daniel J. Decman, Jessie A. Jackson, Richard T. Bernardi, and David C. Camp, "Nondestructive Assay Using Active and Passive Computed Tomography," presented at the INMM 39th Annual Meeting, Naples, FL, July 26-30, 1998.

[SHE82] L. A. Shepp and Y. Vardi, "Maximum Likelihood Reconstruction for Emission Tomography," IEEE Transactions on Medical Imaging, Vol. MI-1, No. 2, October 1982.

[SOM95] J. R. Somoza, H. Szöke, D. M. Goodman, P. Béran, D. Truckses, S.-H. Kim, and A. Szöke, "Holographic Methods in X-ray Crystallography IV. A Fast Algorithm and its Application to Macromolecular Crystallography," Acta Crystallographica, Vol. A51, 1995.

[TAN98] H.R. Tang, "A Combined X-ray CT-Scintillation Camera System for Measuring Radionuclide Uptake in Tumors," Doctoral Thesis, Joint Bioengineering Graduate Group, University of California, Berkeley and University of California, San Francisco, 1998. 


\section{Appendix A}

\section{Reportable TRU Isotopes}

In general, most of the wastes at DOE sites contain primarily weapons grade (WG) plutonium $\left(\sim 94 \%{ }^{239} \mathrm{Pu}\right)$; but some sites have wastes with fuel grade $\mathrm{Pu}\left(\sim 88 \%{ }^{239} \mathrm{Pu}\right)$ and reactor grade $\mathrm{Pu}\left(\sim 80 \%{ }^{239} \mathrm{Pu}\right)$. More importantly, selected sites have wastes with other actinides besides WG Pu. Examples are wastes containing heat source $\mathrm{Pu}$ (highly enriched ${ }^{238} \mathrm{Pu}$ ) at Mound, SRP, PNL, and LANL; wastes with enhanced amounts of 241,3 Am (RFETS, INEEL); wastes with unusual uranium isotopic content (ORNL, INEEL, RFETS); and sites with trans-plutonium wastes (ORNL, LBNL, LANL). Thus, it is incumbent upon the A\&PCT technology to measure a wide range of gamma rays and isotopics. In addition, the Carlsbad Area Office (CAO) Waste Isolation Pilot Plant (WIPP) Waste Acceptance Criteria ((WAC) may require that many of these isotopes be reported in the waste drum characterization. In addition, such isotopic results must be available to the several neutron-based NDA techniques to characterize TRU wastes.

It is likely that few, if any, quantitative measurements can be determined for gamma rays below $120 \mathrm{keV}$ (the famous $100-\mathrm{keV}$ region plus the $K_{\alpha}$ and $K_{B}$ $\mathrm{U}$ and $\mathrm{Pu} \mathrm{x}$-rays). These lower energy emissions will generally be highly absorbed by either the waste matrix or the waste drum, or both. Whether the $129-\mathrm{keV}$ gamma ray of ${ }^{239} \mathrm{Pu}$ can be used quantitatively (for lump correction purposes or otherwise) remains to be determined. The 59.53-keV gamma ray of ${ }^{241} \mathrm{Am}$ can only be used as a flag or qualitative indicator of the presence of americium, since most HPGe detectors used for drum analyses will use cadmium or indium prefilters. However, gamma rays lying in the 140- to $210-\mathrm{keV}$ region should be quantitatively analyzed as often as possible.

The selected gamma rays shown for the isotopes listed in Tables A-1 and A-2 are those recommended to be looked for in every gamma-ray spectrum acquired by A\&PCT. Not all of these isotopes will be present in every waste drum, yet limits must be set on their presence in the waste in order for the neutron-based NDA technologies to correctly interpret their results. Table A-1 lists those TRU isotopes expected to be found in most of the wastes throughout the DOE's waste sites. Table A-2 lists those isotopes that may be found at fewer DOE's waste sites. Depending on where the A\&PCT technology is deployed, those isotopes listed in the second table may need to be the isotopes of interest. The tabular listing suggests that the gamma-ray software search for those listed in Table A-1 first; and then if none of these are found, the code would continue to search for those listed in Table A-2. Finally, for those isotopes listed only the more intense and important gamma rays are shown; their branching ratios are found elsewhere in standard references. 
Table A-1 Primary radioactive isotopes of interest and their gamma-rays

\begin{tabular}{|c|c|c|c|c|c|}
\hline $\begin{array}{c}\text { Energy } \\
\text { (keV) }\end{array}$ & Isotope & $\begin{array}{c}\text { Energy } \\
\text { (keV) }\end{array}$ & Isotope & $\begin{array}{c}\text { Energy } \\
(\mathrm{keV})\end{array}$ & Isotope \\
\hline 129.28 & & 160.31 & & 766.60 & \\
\hline 203.52 & & 642.33 & $240 \mathrm{Pu}$ & 1001.03 & $238 U$ \\
\hline 332.8 & ${ }^{239} \mathrm{Pu}$ & 687.56 & & & \\
\hline \multicolumn{6}{|l|}{344.96} \\
\hline 375.02 & & 208.0 & & 143.76 & \\
\hline \multirow[t]{3}{*}{413.69} & & 335.43 & & 163.35 & ${ }^{235} \mathrm{U}$ \\
\hline & & 368.68 & ${ }^{241} \mathrm{Am}$ & 185.72 & \\
\hline & & 662.42 & & & \\
\hline 148.56 & & 721.96 & & 311.90 & \\
\hline 159.96 & $241 \mathrm{Pu}$ & & & 340.47 & $237 \mathrm{NP}$ \\
\hline 164.58 & 241 Pu & 152.70 & & 415.75 & \\
\hline 208.0 & & 742.77 & & & \\
\hline 267.54 & via & 766.35 & $238 \mathrm{Pu}$ & & \\
\hline 332.35 & & 786.03 & & 661.62 & ${ }^{137} \mathrm{Cs}$ \\
\hline 370.93 & ${ }^{237} \mathrm{U}$ & 1001.03 & & & \\
\hline
\end{tabular}

Table A-2 Rarely encountered radioisotopes and their gamma-rays

\begin{tabular}{|l|l|l|l|l|l|}
\hline $\begin{array}{c}\text { Energy } \\
(\mathbf{k e V})\end{array}$ & Isotope & $\begin{array}{c}\text { Energy } \\
\mathbf{( k e V )}\end{array}$ & \multicolumn{1}{|c|}{ Isotope } & $\begin{array}{c}\text { Energy } \\
(\mathbf{k e V})\end{array}$ & Isotope \\
\hline 146.38 & & 152.63 & & 252.88 & \\
\hline 164.60 & & 817.9 & ${ }^{244} \mathrm{Cm}$ & 333.44 & ${ }^{249} \mathrm{Cf}$ \\
\hline 291.34 & $233 \mathbf{U}$ & & & 387.95 & \\
\hline 317.15 & & 133.0 & & & \\
\hline 320.54 & & 174.0 & $245 \mathrm{Cm}$ & & \\
\hline & & & & 176.6 & \\
\hline 456.00 & & & & 285.0 & $251 \mathrm{Cf}$ \\
\hline 508.00 & $234 \mathrm{U}$ & 346.0 & & & \\
\hline 584.00 & & 402.4 & $247 \mathbf{C m}$ & & \\
\hline
\end{tabular}

